University of Louisville

ThinkIR: The University of Louisville's Institutional Repository

$8-2012$

\title{
Teacher motivation as an enhancement to the first step to success early intervention program for children with tertiary level behavioral challenges.
}

Jon Lee

University of Louisville

Follow this and additional works at: https://ir.library.louisville.edu/etd

\section{Recommended Citation}

Lee, Jon, "Teacher motivation as an enhancement to the first step to success early intervention program for children with tertiary level behavioral challenges." (2012). Electronic Theses and Dissertations. Paper 805.

https://doi.org/10.18297/etd/805

This Doctoral Dissertation is brought to you for free and open access by ThinkIR: The University of Louisville's Institutional Repository. It has been accepted for inclusion in Electronic Theses and Dissertations by an authorized administrator of ThinkIR: The University of Louisville's Institutional Repository. This title appears here courtesy of the author, who has retained all other copyrights. For more information, please contact thinkir@louisville.edu. 
TEACHER MOTIVATION AS AN ENHANCEMENT TO THE FIRST STEP TO

SUCCESS EARLY INTERVENTION PROGRAM FOR CHILDREN WITH

TERTIARY LEVEL BEHAVIORAL CHALLENGES

\author{
By Jon Lee \\ B.A., University of Northern Colorado, 1987 \\ M.A., University of Northern Colorado, 1993

\begin{abstract}
A Dissertation
Submitted to the Faculty of the

College of Education and Human Development of the University of Louisville in Partial Fulfillment of the Requirements

for the Degree of
\end{abstract}

Doctor of Philosophy

College of Education and Human Development

Department of Teaching and Learning

University of Louisville

Louisville, Kentucky

August 2012 
TEACHER MOTIVATION AS AN ENHANCEMENT TO THE FIRST STEPS TO SUCCESS EARLY INTERVENTION PROGRAM FOR CHILDREN WITH TERTIARY LEVEL BEHAVIORAL CHALLENGES

\author{
By Jon Lee \\ B.A., University of Northern Colorado, 1987 \\ M.A., University of Northern Colorado, 1993
}

A Dissertation Approved on

June 13, 2012

by the following Dissertation Committee:

Dr. Kathleen Rudasill, Committee Co-chair

Dr. Andy Frey, Committee Co-chair

Dr. Wendy Reinke

Dr. Amy Lingo

Dr. Maggie McGatha 


\section{DEDICATION}

This dissertation is dedicated to my wife, and children

Mrs. Jennifer S. Elliott Lee,

Ms. Jalen T. Lee, and

Ms. Jordan A. Lee

who reside, most comfortably, at the center of my life. 


\section{ACKNOWLEDGEMENTS}

I would like to thank Dr. Andy Frey and Dr. Kathy Rudasill for their guidance and direction as I muddled my way through this dissertation, and Dr. Wendy Reinke who's original development of the Classroom Check-up made this dissertation possible. Thank you to Drs. Amy Lingo and Maggie McGatha for their thoughtful editing and concise feedback, and to Dr. Tori Molfese. Many thanks to my fellow interventionists, Allyson Rutledge, Pam Ratcliffe, and our research manager Tara Korfhage who are three of the most generous and positive individuals one could ever hope to work with. Undoubtedly, the most genuine and generous scholars that I may ever cross paths with during my career, the following individuals from the Oregon Research Institute and the University of Oregon provided much needed sanction, guidance and support during my work; Drs. Hill Walker, John Seeley, Annemieke Gollly, Ed Feil \& Jason Small. Most importantly, my wife Jennifer and our children Jalen and Jordan deserve much of the credit for this dissertation, as they are my greatest accomplishments in life, and any other goals I have are only attained with their support.

Further Acknowledgement. The Institute of Education Sciences, US Department of Education Grant R324A090237 to the University of Louisville supported the development of this dissertation, in part. The opinions expressed are those of the author and do not represent views of the Institute or the US Department of Education. 


\section{ABSTRACT \\ TEACHER MOTIVATION AS AN ENHANCEMENT TO THE FIRST STEPS TO \\ SUCCESS EARLY INTERVENTION PROGRAM FOR CHILDREN WITH \\ TERTIARY LEVEL BEHAVIORAL CHALLENGES}

Jon Lee

June 13, 2012

The First Step to Success early intervention program (Walker, 1998) is a secondary prevention intervention that targets primary grade children with moderate or emerging behavior disorders. While the effectiveness of the First Step to Success early intervention program has been documented repeatedly (see Loman, Rodriguez, \& Horner, 2010; Walker et al., 2009), it has also been shown to be less effective with more severely disordered children and has a less dramatic impact on behavior in the home than in the school setting. Efforts to enhance the program's effectiveness with even the most severely behaviorally disordered children have been undertaken, and completed. This research collaboration between the Oregon Research Institute and the University of Louisville examined the utility and feasibility of enhancements to the home and classroom components of the First Step to Success intervention. These enhancements, which rely heavily on the infusion of Motivational Interviewing (Miller \& Rollnick, 2002) practices, broadened the ecological focus of the intervention and produced significant changes in the participating children and their families. The following dissertation examines enhancements focused on the classroom teacher's use of praise to 
help replace the intervention's systematic use of external reinforcers; and to reduce the attention for inappropriate behavior (reprimands) that often inadvertently maintains the challenging behavior teachers seek to eliminate. The resulting enhancement, hereafter referred to as the First Step Classroom Check-up, is largely based on the original work of Reinke, Lewis-Palmer, and Merrell (2008). An open multiple-case-study design (Meyers, Truscott, Meyers, Varjas \& Collins, 2007) was used to investigate the intervention for the purpose of innovation and development. The observed increase in teachers' use of praise and decreased reprimands, along with overall positive responses in terms of the interventions social validity, and positive child outcomes provide support for the integration of the Classroom Check-up (Reinke et al., 2008) into an Enhanced version of the First Step to Success Early Intervention Program. These outcomes also demonstrate the promise of future investigations of these interventions separately, and as combined and the probability that the efficacy of the intervention could be investigated. 


\section{TABLE OF CONTENTS}

PAGE

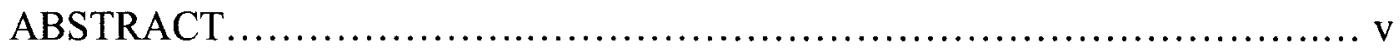

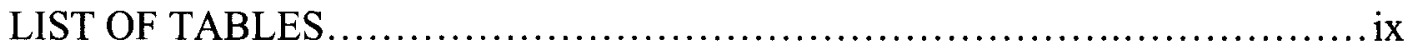

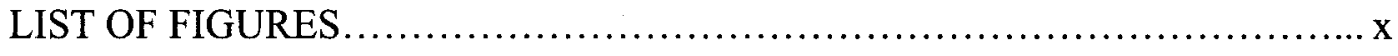

I. STATEMENT OF THE PROBLEM...................................... 1

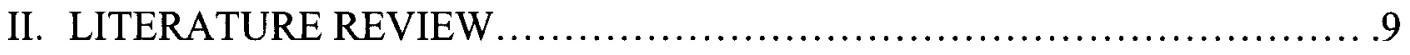

. Theoretical Foundations........................................... 10

. Interventions.................................................. 31

. Social Validity and Implementation Integrity ......................52

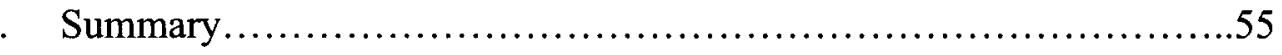

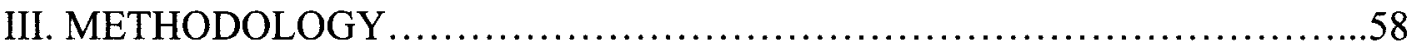

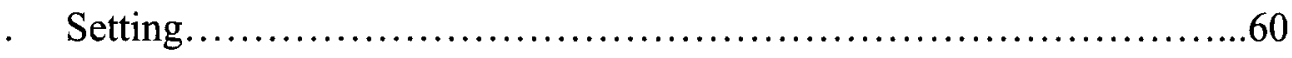

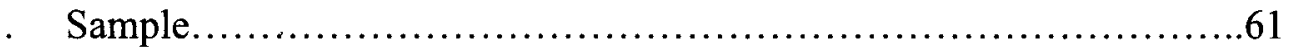

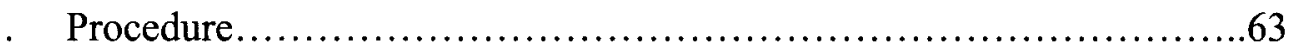

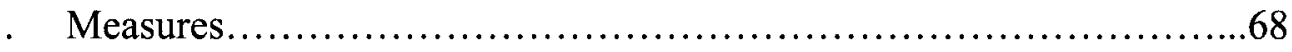

. Analyses.............................................................. 76

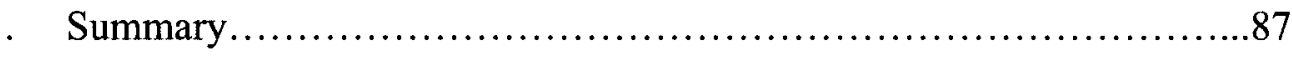

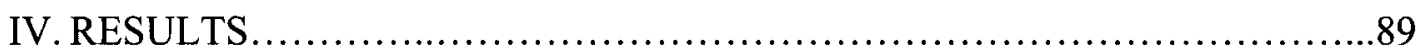

Recruitment and Screening ........................................ 89

Sample...........................................................

Implementation Fidelity .......................................99 


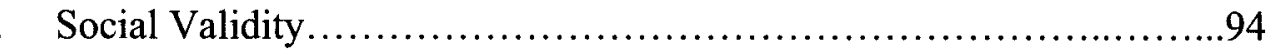

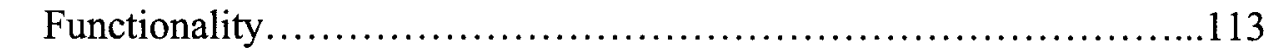

i. Case-level Analysis..........................................113

ii. Composite-level Analysis..................................134

iii. Composite Logic Model Analysis...........................140

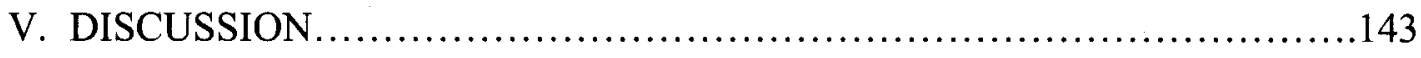

. Implementation Fidelity .........................................145

. Social Validity .................................................... 146

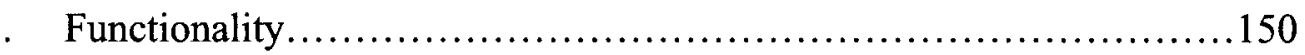

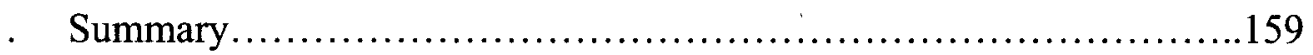

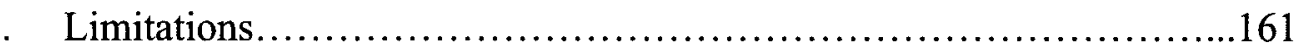

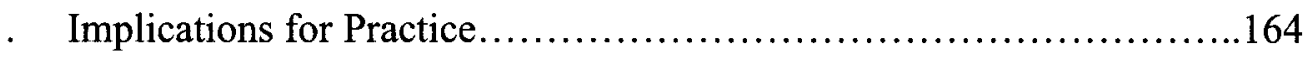

. Implications for Research.......................................166

. Conclusion.................................................... 172

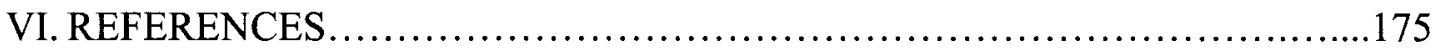

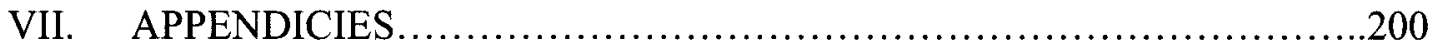

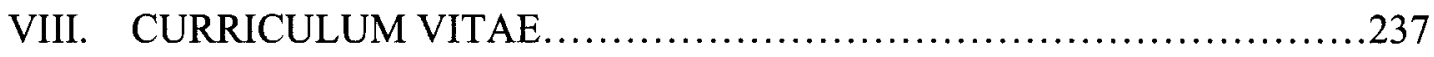




\section{LIST OF TABLES}

TABLE $\quad$ PAGE

1. The Four Steps of the First Step Classroom Check-up.......................65

2. Self-selected Intervention Options for Teachers..........................67

3. MITI Code Summary Score Thresholds................................78

4. The First Step Classroom Check-up Analysis Classification System.........86

5. School-level Summary of Screening Data..............................91

6. Sample Characteristics..........................................92

7. Motivational Interviewing Implementation Quality ....................93

8. Interobserver Reliability for Observations of Teacher Behavior.............136

9. Within-subjects Analysis; Observation of Teacher Behavior Categories.....137

10. Child Intervention Outcomes...........................................139

11. Within-subjects Analysis; Social Skills and Problem Behaviors..............140 


\section{LIST OF FIGURES}

FIGURE

PAGE

1. Model of Interpersonal Social-Behavioral Competencies Within the School Setting .........................................................

2. The First Step Classroom Check-up Logic Model..........................60

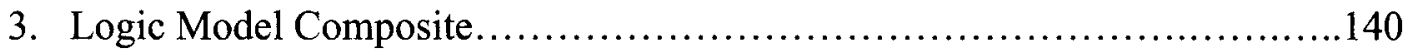




\section{CHAPTER I}

\section{STATEMENT OF THE PROBLEM}

Children who arrive in school today without the social and behavioral skills necessary to succeed face a myriad of challenges, and this lack of preparedness may compound over time, increasing the likelihood of failure not only in academics but also in areas of social and psychological development (Adelman \& Taylor, 2007; Benner, Nelson, Allor, Mooney, \& Dai, 2008). When compared with their peers, children with serious school adjustment and behavior problems demonstrate difficulty understanding social behaviors and cues in the classroom and on the playground (Peppler, Craig, \& Roberts, 1998). Many demonstrate more aggressive intentions towards peers and teachers during free play (Walker, Colvin, \& Ramsey, 1995) as well as more negative, aggressive behaviors in general (Walker, Shinn, O’Neill, \& Ramsey, 1987).

Academically, children with serious school adjustment and behavior problems not only struggle with self-regulation but also with common classroom-related skills, such as positive interactions (listening, sharing, cooperating), attending to instruction, and engaging in academic tasks (Walker, Ramsey, \& Gresham, 2004). These behaviors may lead to rejection by their peers and debilitating cycles of social and emotional failure (Moffit, 1993; Reid, 1993). Frequently, this trajectory leads to detrimental outcomes later 
in the child's life including affiliation with disruptive peer groups, juvenile delinquency, truancy, and school dropout (Patterson, Reid \& Dishion, 1992).

Children whose serious school adjustment and behavior problems persist, become deleterious to their own achievement, the achievement of their peers, and that may result in long term mental health or psychiatric disorders is of great consequence for schools, and a growing concern for families. In fact, according to the 1999 Surgeon Generals Report on Mental Health and the 2000 Report of the Surgeon General's Conference on Children's Mental Health, 1 in 5 children and adolescents have emotional or behavioral problems sufficient to warrant a mental health diagnosis. According to the Center for Disease Control the emotional and behavioral difficulties of children are among the leading health concerns of U.S. parents. In 2005-2006, 8.3 million children (14.5\%) aged 4-17 years had parents who talked with a health care provider or school staff member about the child's emotional or behavioral difficulties, and 2.9 million children were prescribed medication for these difficulties (Simpson, Cohen, Pastor, \& Reuben, 2008). According to the National Center for Children in Poverty (Stagman \& Cooper, 2010) "children and youth with mental health issues in preschool and elementary school are more likely to experience problems at school, be absent, or be suspended or expelled than are children with other disabilities" (p. 4). The number of children whose lives include such adverse factors as parental drug and alcohol abuse; family dysfunction; poverty and unemployment; marital discord; and critical, harsh, and ineffective parenting (Beauchaine, Webster-Stratton, \& Reid, 2005; Bernal, 1984) further compounds these startling statistics. 
Intervening in the lives of these children is important, and the consensus in the literature supports acting early (DeRosier, Cillessen, Coie, \& Doge, 1994; Greenwood, \& Delquadri, 1995; Kazdin, 1987; Patterson et al., 1992; Reid, 1993; Reid \& Eddy, 1997; Walker et al., 1996). A review of research in the treatment of delinquency in adolescence by Zigler and Taussig (1992) concluded that early intervention was the single most effective strategy available for the prevention of later delinquency. Two important scholarly reviews took up this mantle (See Greenberg, Domitrovich, \& Bumbarger, 1999; Leff, Power, Manz, Costigan, \& Narbors, 2001) and provide information regarding the characteristics of effective interventions. Across both reviews, effective interventions were found to (a) engage multiple intervention agents (e.g., parents, teachers, and interventionists); (b) be applied for at least one school year; (c) utilize multiple components (e.g., training for parents and teachers, direct intervention with the child); and (d) include multiple settings (e.g., home, school, community).

More recently, Hoagwood et al. (2007) reviewed articles published between 1990 and 2006 on school-based mental health interventions. Using stringent methodological criteria for inclusion, these authors found only a limited number of articles that focused on mental health and educational outcomes. Research demonstrating positive effects for children's mental health and education provided intervention components in the home and school, targeting parents and teachers. Interventions that demonstrate mental health benefits, but not educational impact, tended to lack intensity, and had little or no family involvement. Hoagwood and her associates help build the case that the inclusion of both school (teacher) and home (parent) intervention components may be necessary to be effective in preventing and treating the issues associated with serious school adjustment 
and behavior problems. Of the interventions considered across each of these three reviews, the First Step to Success early intervention program (Walker, 1998) was mentioned most favorably, and as an exemplar (Greenburg, et al., 1999; Hoagwood, et al., 2007; Leff et al., 2001).

First Step to Success is an early intervention program designed for at-risk elementary school children in the primary grades who show clear signs of emerging externalizing behavior patterns including aggression toward others, oppositional-defiant behavior, tantruming, rule infractions, and escalating confrontations with peers and adults (Walker, et al., 1997). The at-risk child is the primary focus of the First Step to Success program, and hereafter is called the focus child. Teachers, peers, and parents participate in the intervention as implementation agents under the direction and supervision of a trained First Step behavior coach (hereafter called the First Step coach) who is frequently a related service provider (e.g., school counselor, social worker, special educator), and has overall responsibility for coordinating the intervention. The First Step to Success early intervention program requires two to three months from start to finish, and is applied to only one focus child at a time in regular or special education classroom settings.

First Step consists of three components designed to be applied in concert with each other: (a) a multiple-gating universal screening process; (b) a school module (including an adapted version of the Contingencies for Learning Academic and Social Skills program; CLASS; developed by Hops et al., 1978) referred to hereafter as First Step CLASS component; and (c) a home component called homeBase. The two primary goals of the First Step program are to teach the focus child to get along with others 
(teachers and peers) and to engage in assigned schoolwork in an appropriate, successful manner. The three modules of First Step are based on extensive research on school and home intervention procedures with aggressive, antisocial youth and over a decade of work related to the universal, proactive early screening of at-risk children to provide early detection (see Hops \& Walker, 1988; Patterson, et al., 1992; Walker, et al., 1998).

The First Step to Success early intervention program was initially developed in 1992 and has been extensively evaluated using multiple research designs, including single subject (Carter, \& Horner, 2007, 2009; Golly, Sprague, Walker, Beard, \& Gorham, 2000; Overton, McKenzie, King, \& Osborne, 2002; Sprague, \& Perkins, 2009), longitudinal (Nelson et al., 2009; Walker et al., 1998), quasi-experimental (Diken, \& Rutherford, 2005; Golly, Stiller, \& Walker, 1998), and experimental (Walker et al., 2005; Walker et al., 2009). These evaluations have demonstrated strong, positive classroom effects across the majority of the at-risk primary level elementary school children with moderate or emerging behavioral disorders that were treated. Furthermore, the First Step to Success program demonstrates social validity across a variety of applications (Golly, Stiller, \& Walker, 1998; Walker, et al., 1998) and high levels of treatment implementation fidelity (Walker et al., 2009).

Unfortunately, for children whose challenging behavior is severe, the original First Step to Success early intervention program (Walker, 1998; Walker et al., 1997) has been less effective in two distinct areas. First, the results indicate a less dramatic impact on behavior in the home than the school setting. Second, First Step to Success is generally not sufficient to substantially decrease problematic behavior or to increase adaptive behavior of the most severely disordered students (Walker et al., 2009). Many of 
the positive gains children demonstrate in the classroom immediately following the implementation of the First Step program tend to fade without the use of continued monitoring and booster sessions once the intervention is discontinued (See final IES report for: Evidence-based Interventions for Severe Behavior Problems-First Step to Success, March, 2010). Researchers from the Oregon Research Institute and the University of Louisville are currently working to augment the current version of the First Step to Success early intervention program to address these concerns.

An important component in the success of the CLASS component of the First Step to Success early intervention program (Walker, 1998) is its focus on professional development for the teacher. The primary focus of professional development activities is to promote positive interactions and minimize negative interactions between the focus child, and his or her teacher and peers. To this end school staff are introduced to the Five Universal Principles of Positive Behavior Support (Golly, 2006): 1) Define expectations; 2) Teach expectations; 3) Reinforce expectations; 4) Minimize attention for minor inappropriate behaviors; and 5) Have clear consequences for unacceptable behavior. These principles establish a set of baseline classroom expectations within which the First Step CLASS component is more likely to have a positive impact on the focus child and the child's peers, and are infused into all professional development activities (See also, Buyse, Verschueren, Doumen, Van-Damme, \& Maes, 2008).

A possible explanation why increased social skills and decreased problem behaviors fade after the program is discontinued involves the program's existing preservice training, intervention prompts, coaching (See Rodriguez, Loman \& Horner, 2009) and consultation procedures to support the teacher's implementation of the 
program. It is possible that these procedures are not implemented with enough integrity to sustain teachers' use of praise with the focus child as the use of external reinforcers are systematically faded near the end of the intervention. Although teachers' use of praise with the focus child has yet to be systematically identified as a cause of the diminishing classroom effects of the First Step program, it has recently been reported as a collateral benefit for focus children and peers in classrooms where the First Step program is implemented (Sprague \& Perkins, 2009). Additionally, teacher's use of praise is represented as one of a twenty-item fidelity measure utilized to examine the teacher's implementation of the First Step CLASS component in the classroom. This item ("Does the implementer provide the child positive feedback during the game?") asks for a dichotomous rating of implementation (yes, no) and a rating of quality of the implementation on a five point Likert scale (very poor to excellent). However, this single item is not likely to discriminate between teachers well. A comprehensive understanding of the teacher's implementation integrity is imperative in order to infer intervention effectiveness (Sheridan, Swanger-Gagné, Welch, Kwon, \& Garbacz, 2009), and therefore critical to improving student outcomes.

This proposal represents initial efforts to evaluate the feasibility and acceptability of an intervention to enhance teachers' use of praise in order to address the concerns identified above. The intervention is based on the counseling approach of Motivational Interviewing (MI). Miller and Rollnick (2002) define MI as "a client-centered, directive method for enhancing intrinsic motivation to change by exploring and resolving ambivalence" (p. 25). Building on the original work of Reinke et al. (2008), an enhancement to the First Step CLASS component, the First Step Classroom Check-up 
(FSCCU), was developed and pilot tested. The FSCCU incorporates processes modified from this work to (1) engage the teacher in a working alliance; (2) measure the teacher's use of praise and reprimands in the classroom; (3) use this data to provide teachers performance based feedback and motivation for change; and (4) when rates of negative feedback are found to be high, to provide extended consultation, education and support. The purpose of this study (which exists within the larger IES grant - Enhanced First Step to Success) was to develop measurement protocols, training materials and implementation procedures infusing the Classroom Check-up (Reinke et al., 2008) into the First Step CLASS component. It is believed that teacher behavior change, a possible mediating variable in the intervention process, will more likely be sustained into the teacher and maintenance phases of the First Step CLASS component, and therefore increase the potential for sustaining the effects of the program after fading of external reinforcers. To this end an iterative process of pilot testing and refinement of the measurement protocols, training materials and implementation procedures of the FSCCU component was undertaken to (a) determine if the intervention was implemented with fidelity, (b) understand if key stakeholders perceived the intervention as socially valid, (c) better understand if the intervention functioned as intended. 


\section{CHAPTER II \\ LITERATURE REVIEW}

Chapter two contains two distinct sections. The first frames the theoretical foundations of this dissertation. Three primary areas shape the theoretical foundations of this literature review, beginning with the ecological systems theory of Uri Bronfenbrenner (See Bronfenbrenner, 1986; 1989; Bronfenbrenner \& Morris, 2006) within which risk, protective, and promotive factors are defined, and the independent and dependent variables associated with this dissertation are organized. Next, an introduction to coercive home and classroom transactions sets the stage for the importance of teacherchild relationships.

The second section of this literature review examines interventions that reflect the contextual nature of this proposal's combined intervention effort: (1) the counseling approach known as Motivational Interviewing (Miller \& Rollnick, 2002); (2) the Parent Motivation Inventory (Nock \& kazdin, 2005); (3) the Ecological Approach to Family Interventions and Treatment (EcoFIT; Dishion \& Stormshak, 2007); the Classroom Check-up (Reinke et al., 2008), a professional development system for teachers; and, the First Step to Success early intervention program (Walker, 1998). Finally, the importance of social validity and treatment integrity in the development of interventions is briefly reviewed. 


\section{Theoretical Foundations}

Ecological Systems. Biological and ecological factors influence children's developing school adjustment and behavior. Taken at face value, it is simplest to view a child's growth and development as contingent on the interactions that occur within the child's immediate proximity. However, the ecological model invites consideration not only of the processes as directed exclusively at the child but also (a) between those who are located within an intimate sphere of influence, (b) from those just outside this sphere, (c) from those who reside distally in the wider world, (d) and throughout time.

In an ecological view, simple interactions become complex. An interaction becomes transactional when considered withtn the ongoing processes of the child's ecology. For example, even a singular interaction occurring amongst the most distal contexts of a child's ecology will create currents of influence throughout the entire system. These currents flow in all directions and reach each member of the child's ecology, to one degree or another, including the child. Based upon an ecological model (See Bronfenbrenner \& Ceci, 1994), a transactional model of child development (Sameroff \& Fiese, 2000; Sameroff \& MacKenzie, 2003) acknowledges that interactions are more appropriately defined as transactions, and occur between the child, the child's biological heritage (inheritance), and the environment. According to Sameroff and Fiese (2000) "In this approach developmental outcomes are a function of neither the individual

alone nor the experiential context alone. Outcomes are a product of the combination of an individual and his or her experience" (p. 10).

Consider the child's response to any experience within their intimate sphere of influence not as an end to a didactic exchange, but rather as a transaction that continues 
the interplay between contextual elements - responding to the child's response and so on, dialectically (Sameroff \& MacKenzie, 2003). Atypical responses by any one element or between elements of our ecological model affect all other elements (to some extent) and will eventually influence the child's development. Similarly, when you drop a stone into a pond of water the subsequent ripples reach (and thus influence) even the most distal elements of the pond. We might envision the transactional nature of this event if we could see each element respond with ripples of their own, reaching out to all other elements and eventually returning to the point where the stone entered the water.

Utilizing Bronfenbrenner's ecological system (1979), variables important to our work with teachers and young children through this research proposal can be located as we might locate various points on a map. The immediate environment of the child, the microsystem, consists of the physical, social and psychological experiences (Swick \& Williams, 2006) and intimate relations that support the child's earliest and most profound learning. This word's Greek origin can help us better understand the term. The combining form 'micro' (in English used as a prefix) means small or limited, and 'system' a derivative of sústēma means (literally) brought together. Typically a small, intimate group of people, a child's family constitutes the key membership of the microsystem. However, the microsystem is not static. As the child grows and develops the microsystem expands and moves to include new environments like childcare centers, school classrooms, and even neighborhoods (e.g., Garbarino, 1992; Rogoff, 2003). Members of these new environments who, like the family, are intimately related to the child (e.g., caregivers, teachers, peer groups) are brought together within the newly expanded sphere of influence. Social skills and abilities learned from the family may come to bear within 
these new environments, and often to a parent's chagrin - vice versa. The relative influence and significance of various microsystem elements is thought to shift over time (Sameroff \& MacKenzie, 2003). For example, as the child enters adolescence, peer groups and the environments in which they interact exert strong influence on the child's socio-behavioral development (Dishion, McCord, \& Poulin, 1999; Garabarino, 2001).

The microsystem is a rich source of influence from which the child learns about the world, yet it becomes more complex when the transactions between the constituent members of the microsystem are considered. Bronfenbrenner (1979) labeled these relations as the mesosystem. Also Greek, the combining form 'meso' (from mésos) is used as a prefix in English to describe the middle or in between. For example, the relationship between a child's teacher and parent, who both influence the child independently of one another from within their respective school and home environments, also influence the child more subtly through their transactions with each other (i.e., in the mesosystem). As the child matures and becomes entrenched in his or her peer group during adolescence, any negative relations between parent and peers might work to strain the relationship, and is another example of mesosytem influence on the child. Although mesosytem transactions are logical, the differing impact on the child from within the mesosystem is complex, multifaceted, and difficult to measure.

Next in Bronfenbrenner's nested system of influence is the exosystem, which represents the influence of environments that the child may not be a member of physically but is affected by nonetheless. This word's Greek prefix, 'exo,' means outside. As an example, one could postulate that the culture, stress level, and system of rewards (social and financial) in the parent's work environment (or those conditions that stem 
from a lack of work) influences the stress level and social capacity of the parent when interacting with the child and/or the child's teacher. In this example, an environment from the parent's microsystem exerts influence within the micro- and mesosystems of the child. The same may be true for the influence of a school's climate or culture on the instructional and disciplinary practices of a teacher. Although the child is not physically present in the parent's workplace, or at the school faculty meeting, these environments influence the child from a more distal position than the micro- or mesosystem. Indeed, further still from the child are the macro- (from the Greek combining form macros, meaning long) and chrono- (a derivative of the Greek word khronos, meaning time) systems. The macrosystem consists of the cultural, societal and political influences at work in world, and "act[s] as a powerful source of energy in our lives" (Swick \& Williams, 2006, p. 372). The passage of Public Law 107-110 of 2002 (the No Child Left Behind Act; NCLB) provides an appropriate example of influence from the macrosystem as it ripples through each system until eventually reaching the child. The NCLB act increased accountability for children's achievement for school districts across the country. Increased accountability had the effect of narrowing the scope and depth of school district curriculums so as to better align with standardized forms of assessment, thus impacting scope and depth of a child's learning (Renner, 2010). As well, NCLB employed a contracted definition of scientifically based research, distressing teachers who utilized developmentally appropriate practices that lacked a scientific empirical base, affecting children through the changes in instructional practices and teacher's stress associated with this endeavor. 
The final system of the ecological model is that of the chronosystem, which represents the historical context of our lives. For example, children who are removed from their homes face negative effects that may peak during the first year of their placements in foster systems, but that may stabilize over time if the placement remains constant. Also represented by the chronosystem are the greater sociohistorical contexts of our lives, including the long-term effects of the recent economic decline or the events that have shaped a generation of children - like the events of September 11, 2001.

From within and amongst the personal ecologies of the teachers and children that are the focus of this study, we can identify risk, protective and promotive factors that work to support or degrade the social-behavioral competencies necessary for children to succeed and flourish.

Risk, Protective, and Promotive Factors. Jenson and Fraser (2006) define risk factors as those "...individual, school, peer, family and community influences that increase the likelihood of such problem behavior as dropping out of school or becoming a juvenile delinquent" (p. 5), while protective factors are those influences that counteract risk and work to reduce its effects (Richman \& Fraser, 2001). Promotive factors (unlike protective factors, whose influence against risk is thought to occur only in the presence of risk) may influence positive developmental outcomes independent of risk (Fraser, 2004).

Important to the discussion of risk, protective and promotive factors and the vulnerability or resilience of children to the influences of each, is the recognition of the interplay between these factors (and others), and the relative strength of each as they exert influence on the developing child (Jenson \& Fraser, 2006). Masten (1987) posits two models for understanding children's differential responses to risk. The first, an 
additive model, conceptualizes risk and protection on opposite sides of a continuum measuring the probability of poor adaptation (more risk) and positive adaptation (more protection), not unlike a set of balance scales measuring the amount of risk and protection in relation to the other.

For example, a kindergartener's prideful grin may be dependent on the teacher's praise for his or her hard work (a positive interaction), while a sharp reprimand from the teacher is likely to produce an equally mordant negative response from the child (a negative interaction). These interactions demonstrate a simple dependency between the teacher's praise or reprimand and the child's response, and are easily accounted for using an additive conceptualization - similar to the idea of cause and effect. Unfortunately, the additive model does not account for the differential effects of risk when considered from a transactional perspective. For example, a child with serious school adjustment and behavior problems may react defiantly to teacher's praise. The child's defiance escalates the tenor of the teacher's reaction, who responds harshly providing an illogical consequence for the child's defiance. The differential effects of the child's behavior problems varied the child and teacher's reactions, in this case qualitatively, from the typical dependency seen in the previous examples.

Masten (1987) describes an interactive model to better account for the effects of protective and promotive factors (e.g., teacher praise) in the presence of or absence of risk (e.g., serious school adjustment and behavior problems). In this model, protective and promotive factors may buffer against risk, disrupt the pathways which multiple risk factors work through, or prevent the initial occurrence of risk. To exemplify this model we return to our previous example, in which the teacher was caught off guard by the 
child's reaction to praise. This time our teacher acts in an emotionally supportive fashion, altering the delivery of praise to the child with serious school adjustment and behavior problems in order to match the child's expected temperamental reaction. Thus, the teacher may have tempered his or her own reaction to the child's defiance, showing an understanding of the child's challenges - possibly even choosing to approach the child's defiance calmly, at a later time. Conceptualizing risk, protective, and promotive factors with an interactive model provides the framework from which to better understand the moderating role of various protective and promotive factors differentially, particularly from within the ever changing ecological systems and transactions of classroom environments (Fraser, 2004).

Figure 1: Model of Interpersonal Social-Behavioral Competencies within School Settings

\begin{tabular}{|c|c|}
\hline \multicolumn{2}{|c|}{ Teacher-Related Adjustment } \\
\hline \multicolumn{2}{|c|}{ Related Behavioral Correlates } \\
\hline ADAPTIVE & MALADAPTIVE \\
\hline -Comply promptly & -Steal \\
\hline -Follow rules & -Defy or provoke teacher \\
\hline -Control anger & -Tantrum \\
\hline -Make needs known approp. & -Disturb others \\
\hline -Produce acceptable work & -Damage property \\
\hline -Work independently & -Cheat \\
\hline -Adjust instructional situations & -Swear or make lewd gestures \\
\hline $\begin{array}{l}\text {-Respond to teacher } \\
\text { corrections }\end{array}$ & $\begin{array}{l}\text {-Aggress towards others } \\
\text {-Ignore teacher }\end{array}$ \\
\hline \multicolumn{2}{|c|}{ Outcomes } \\
\hline POSITIVE & NEGATIVE \\
\hline -Teacher acceptance & -Teacher rejection \\
\hline -School achievement/success & -Referral for specialized \\
\hline & -School failure and/or dropout \\
\hline & $\begin{array}{l}\text {-Low performance } \\
\text { expectations }\end{array}$ \\
\hline
\end{tabular}

\begin{tabular}{|l|l|}
\hline \multicolumn{1}{|c|}{ Peer-Related Adjustment } \\
\hline \multicolumn{1}{|c|}{ Related Behavioral Correlates } \\
ADAPTIVE & \multicolumn{1}{|c|}{ MALADAPTIVE } \\
-Cooperate with peers & -Disrupt the group \\
-Support peers & -Act snobbish \\
-Defend self in arguments & -Aggress indirectly \\
-Remain calm & -Start fights \\
-Achieve much & -Short temper \\
-Lead peers & -Brag \\
-Act independently & -Seek help constantly \\
-Compliment peers & -Achieve little \\
-Affiliate with peers & -Get in trouble with teacher \\
\hline \multicolumn{1}{|c|}{ POSITIVE } & Outcomes \\
\hline Peer acceptance & NEGATIVE \\
\hline -Positive peer relations & -Social rejection/ neglect \\
-Friendships & -Low self-esteem \\
\hline & -Weak social involvement or \\
\hline
\end{tabular}

Walker, Irvin, Noell, and Singer (1992) provide a conceptualization of teacher-

and peer-related systems of social and behavioral competencies, which serve as protective and promotive factors that all children must negotiate in their adjustment to 
schools and schooling (Figure 1). This work grounds the previously discussed theories (ecological systems, transactional models, risk, protection, and promotion) within an education-specific empirically derived model (Lane, Gresham, \& O'Shaughnessy, 2002; see also Walker et al., 1988; Walker et al., 1995).

One competency, particularly relevant in the classroom experience of the child with serious school adjustment and behavior problems, is the lack of opportunities for teachers to notice and praise adaptive behavior. Often, due to the student's maladaptive social-behavioral competencies, negative coercive transactions prevail.

Based on this premise, the First Step CLASS component works to influence the transactional nature of the teacher's relationship with behaviorally challenged children such that maladaptive behavior correlates are reduced, and adaptive ones enhanced, thus leading to positive outcomes in both teacher and peer domains. This research proposal represents an effort to affect change in the teacher, representing the teacher-related side of this model from within an ecologically valid program (i.e., First Step to Success). Specifically, a focus on increasing teacher's use of praise for adaptive behaviors, while reducing the negative or coercive attention for inappropriate behavior that often plagues young children with serious school adjustment and behavior problems.

In the following sections, the theoretical perspectives of coercive home and classroom transactions are reviewed, followed by a brief review of teacher-child relationships and their protective and promotive value in child development.

Coercive Home and Classroom Transactions. Patterson and colleagues have researched the reoccurring family-based cycles of coercion for children with serious adjustment and behavior problems (Moffitt, 1993; Patterson, 1976; Patterson, 2002; 
Patterson \& Reid, 1970; Patterson, Reid, \& Dishion, 1992; Patterson \& Yoerger, 1997). Others have extended the model to include transactions within schools and classrooms between teachers and children (McEvoy \& Welker, 2000; Patterson, Reid, \& Dishion, 1992; Reinke \& Herman, 2002). Below, reciprocity and coercion are defined and exemplified within the context of parent-child and teacher-child interactions.

While positive, nurturing classroom relations are ideal, ineffectual coercive or overly negative classroom management practices may be more prominent in classrooms where children with developing school adjustment and behavior challenges are educated. Jenson, Olympia, Farley, and Clark (2004) suggest that children in today's schools who exhibit challenging externalizing behaviors "exist in a sea of negativity with little possibility for positive educational experiences and personal relationships" (p. 67). This statement seems an exaggeration, but the evidence supports the underlying message. All told, the rates of positive to negative feedback are very low (Scott, Alter, \& Hirn, 2011; Shores, Gunter, \& Jack, 1993) and, as will be described below, may work to maintain cycles of coercion between teacher and student that limit the amount of reciprocity in the classroom. Teachers often self-report themselves as more positive than naturalistic observations have borne out (Nicholas, Olympia \& Jenson, 2001). This contradiction is interesting. It is possible that teachers are not aware of the level of negativity in their classrooms brought on by coercive relationships. In addition to low rates of positivity in classrooms and the contradiction in teacher's perceptions of classroom valence, children with developing school adjustment and behavior problems are prone to more negative transaction with their teachers than positive (Sutherland, \& Oswald, 2005; Sutherland, Wehby, \& Copeland, 2000). Often, negative transactions lead to an escalation in 
oppositional behavior, with the student resisting teacher requests. This process continues with increasing levels of aversive behaviors until, finally the teacher or student relents to the other. The entire process is thought to maintain and even reinforce these coercive cycles for both the teacher and the child. (Maag, 2001; Shores, Gunter, \& Jack, 1993).

Patterson and Reid (1970) define reciprocity as interactions characterized by mutually reinforcing behaviors of a positive nature, which are typically equanimous for those involved. For example, in the following verbatim, both teacher and student are positively reinforced by their interaction. The mutual reinforcement of the transaction supports the continuation of the conversation, and likely the reoccurrence of reciprocity in future communications.

Teacher: "All right then, who has the answer to our first problem?"

Student: "I do! I got 24."

Teacher: "Right you are, terrific!"

Student: "I've also figured out number two!"

Teacher: "Well go on then, let's have it!"

Student: " 14 , it's 14 !"

Teacher: "I am impressed! OK, now it's time to..."

Student: "And number three is..."

Teacher: "Whew! You'd better hold on kiddo, or we'll never make it to music!"

If it were not for the upcoming music class this conversation might go on all day, as both teacher and student were reinforced by the interaction; the teacher was reinforced by the energetic responses to her questions, and the student gained a sense of pride and accomplishment from her correct answers. Reciprocity allows the time and relational space necessary for teachers to provide positive attention and reward for pro-social behavior. However, reciprocity involves the ability to distinguish and respond to the 
social clues of attention and inattention (Patterson \& Reid, 1970). In this example, our lively student did not recognize the teacher's attempts to move on from the conversation. Responding to social clues is a challenging skill, especially for children with serious school adjustment and behavior problems. The inability to recognize these clues may cause frustration for their reciprocal partners, interrupting the rewards necessary for the continuation of this mutually reinforcing interaction.

Alternatively, a coercive model provides positive reinforcement for only one member of the dyad, while the second receives negative reinforcement after capitulating to demands set forth by the other. Coercive transactions in the home are thought to establish the precursors for latter developing antisocial behaviors in the expanding ecologies of children as they mature (e.g., in schools; McEvoy \& Welker, 2000, Patterson, Reid \& Dishion, 1992; Reinke \& Herman, 2002, see also Garabarino, 1992). Pianta, Nimetz, and Bennett (1997) support this hypothesis; "We argue that consistency across child-mother and child-teacher relationships is a function of consistency in children's relational styles, or models of how relationships with adults work" (p. 277). In the following example of a coercive transaction in the home, the child initiates.

Child: "Mom, can I play a video game?"

Parent: "No, honey, I'm sorry, it's too close to dinner. Come and help me prepare the meal."

Child: "You NEVER let me play!"

Parent: "Don't raise your voice at me. We don't have enough time! Anyway, it will be fun to cook together."

Child: "All I want to do is PLAY MY GAME!"

Parent: "No, now don't ask me again."

Child: "NEVER, NEVER, NEVER! I NEVER GET TO PLAY! YOU ARE MAKING ME CRY! 
Parent: "I am really tired..."

Child: "I HATE THIS!"

Parent: "OK, OK, just stop yelling!"

In this example the child is positively reinforced (is allowed to play a video game) for his or her inappropriate behavior, and the mother is negatively reinforced (assuming the interaction is terminated) as she relents in order to stop a temper tantrum. These forms of reinforcement work to maintain the behavior of the child, who is likely to use temper tantrums in the future and the mother who will likely concede to the video game in the future in order to avoid another tantrum. Is it not often the case that we, as parents, use these coercive transactions to justify a change in our parenting values? For example, the parent from the scenario above may later justify allowing the child to play video games rather than help in the kitchen by saying, "it will keep my child occupied while I prepare the meal." In the following example the transaction occurs in the school classroom, with the teacher initiating, and unfortunately ends in a similar fashion.

Teacher: "OK everybody, I need you to get out your writing journals"

Child: "I don't like to write."

Teacher: "Sorry, it's time to finish our stories."

Child: [whining] "No, please let me keep reading."

Teacher: "Not now, come on..."

Child: [crying] "All I want is to read a little longer. I NEVER get to read."

Teacher: "Come on now, you're a good writer."

Child: [intensifies crying, turns head away from teacher and pouts]

Teacher: "OK, OK, stop crying you can read for just a bit longer."

Again, both child and teacher are reinforced: the child positively (reads a bit longer) and the teacher negatively (avoids a tantrum). In either scenario, the mother or teacher could have utilized increasingly harsh language and threats of discipline to reverse the results of 
the transaction. In that case the child's compliance would have positively reinforced the mother or teacher, and avoiding punishment would have negatively reinforced the child.

Coercive transactions are reinforcing such that teachers and children, often unknowingly, become expert coercers. It is the oddly reinforcing nature of the coercive cycle that is misunderstood by teachers, or at least not acknowledged. It is important to recognize for its significance in maintaining ineffectual or overly negative classroom management practices, and thus maladaptive behavior. When played out frequently in the home and classroom, transactions such as these work to condition the participants to respond to adverse behavior, reduce the general positive valence in the environment, and limit the opportunities to provide praise for positive social behavior (Forgatch \& Patterson, 1998 as cited in McEvoy \& Welker, 2000; Patterson \& Reid, 1970), while the inherent reinforcement works to maintain the cycles over time. In the worst situations, those involved could continue to increase the intensity of their reactions to one another, escalating the transaction to the point of extreme emotional outbursts, or the use of physical measures (e.g., elopement, restraint, violence).

The developmental progression of coercive cycles and harsh, ineffectual discipline practices that begin at home can, if left unchecked, reinforce children's developing social and behavioral challenges leading to: alienation from positive prosocial groups at school, intensified anti-social behaviors, and eventually an adolescence which may include delinquency (Garbarino, 2001; Patterson, Reid \& Dishion, 1992; Walker et al., 1987; Walker et al., 2004). Children who have experienced repeated coercive transactions, and thus have developed maladaptive social and behavioral competencies, will find acceptance by their teachers and peers difficult. In essence, these 
children's externalizing behaviors (e.g., defiance, ignoring, tantruming) may result in rejection by their peers and conflictual relationships with their teachers (Birch \& Ladd, 1996; 1997; Walker et al., 1992; see Figure 1). In an interactive conceptualization of risk, protective and promotive factors, deleterious relationships negate the protective role that positive teacher-child relationships have in the presence of risk as well as the promotive function for children's overall development and well-being (Birch \& Ladd, 1996). The ill effects of conflictual relationships are not relegated solely to the child. Teachers experience frustration and burnout that may be related to this coercive cycle (Jennings \& Greenberg, 2009), adding further detriment to the classroom climate overall.

In summary, children who experience coercive family relationships may demonstrate serious adjustment and behavior challenges at school, characterized by peer rejection and conflictual teacher-student relationships. From a developmental perspective, children bring these problematic behaviors to the classroom and utilize them in a similar fashion as they were learned in the home - as a means of control and/or avoidance. Often unknowingly, due to the reinforcing nature of coercive transactions, teachers enter into these coercive relationships to control the child, or as a means of avoiding conflict with the child. In doing so teachers continue the coercive relationships the child experienced at home, or foster in the child the use of coercion as a means of control and avoidance in the classroom. In either case, teachers who tread down this slippery slope limit opportunities to provide praise for positive social behavior and reduce the general positive valence of the classroom, while providing reinforcements that work to maintain the coercive cycles over time. 
While the First Step to Success program demonstrates significant positive results for children with moderate challenges, those children with serious school adjustment and behavior challenges often do not maintain these positive results after the intervention has ended. Infusing the existing First Step CLASS component with a procedure to influence teacher behavior in this regard (the Classroom Check-up; Reinke et al., 2008) is designed to intervene in the coercive relationships that often develop between a teacher and student with serious school adjustment and behavior challenges. Hypothetically, if the intervention is a success (i.e., the teacher responds to the FSCCU intervention and the child to the First Step CLASS component), this change may be measured in the sustained positive effects of the program and the teacher's perception of the teacher-child relationship.

The evidence presented here indicates that children with serious school adjustment and behavior problems arrive at school with challenging behavioral orientations. Next, how these orientations affect children's adjustment to school, and their relationships with peers and teachers are explored.

Teacher-Child Relationships. In a series of well-conceived studies, Ladd, Birch, and Buhs (1999) found evidence that children who demonstrated prosocial behaviors in their transition to school (kindergarten) differed in their relationships with peers and teachers from those who approached this transition demonstrating more anti-social behaviors. These differences affect children's approach to the development of mutual friendships, their acceptance or alienation by peers, and their relationship with teachers 
(closeness or conflict as perceived by the teacher ${ }^{1}$ ). Ladd, Birch, and Buhs (1999) and Ladd and Burgess (1999) found that children whose predominant coping mechanisms were reactionary and aggressive suffered from peer rejection and alienation, and this behavior orientation brought about stressful interactions with teacher (i.e., conflict). However, children whose adjustment to school was more constructive enjoyed peer acceptance and closer relationships with teachers.

These developing relationships have long lasting importance, as demonstrated by Ladd and Burgess (1999) who found that aggressive behaviors and associated classroom effects (i.e., low peer acceptance, conflictual teacher-child relationships) were stable from kindergarten to grade two. Jerome, Hamre, and Pianta (2009), using data from a longitudinal study (NICHD SECC), found teacher ratings of closeness and conflict to be relatively stable from kindergarten to sixth grade. Hamre and Pianta (2001) found that negative teacher-child relationships are predictive of behavioral problems into middle school. The evidence presented here seems to indicate that the trends demonstrated by these children might intensify during the primary school years, and worsen if not intervened with. As suggested by Rudasill (2011), “...the nature of early teacher-child relationships may form a model for children about the way teacher-child relationships should and will be" (p. 148).

Indeed, Jerome et al., (2009) found evidence to support intervening in these relationships early, as teachers' ratings of conflict in the teacher-child relationship increased during the primary grades with the greatest changes occurring between kindergarten and second grade. Furthermore, teacher ratings of closeness decreased at

\footnotetext{
1 Respectful, caring, warm, friendly teacher-child relationships are said to be high in closeness; teacher-child relationships, which are predominately, harsh, angry, and/or coercive are characterized as conflictual (Birch \& Ladd, 1996; 1997; Hughes, Gleason, \& Zhang, 2005; Pianta, 2001).
} 
each of the seven grade levels measured, with the greatest decreases occurring in the later elementary years.

Poor teacher-child relationships may not be defined solely by the child's behavior alone. Koomen, Verschueren, and Pianta (2007; as cited in Split \& Koomen, 2009) found that low levels of competence and job satisfaction, and high levels of teaching stress were related to teachers' identification of poor teacher-child relationships. Furthermore, Pianta, Stuhlman, and Hamre (2002) posit that teacher-child relationships characterized by negativity can erode the later value that positive relationships provide as a developmental resource (p. 94).

The evidence reviewed to this point indicates support for the hypothesis that children's relationships at home provide a foundation for the development of early relationships upon their transition to school. As these early school relationships are fairly stable, predictive of future school adaptation and teacher-child relationships, the need to intervene early in coercive or conflictual teacher-child relationships is evident. However, the coercive processes experienced by some children in their homes prior to school entry are not the sole predictors of conflictual teacher-child relationships. A number of individual characteristics are useful for this purpose and are described next.

Children's individual characteristics are salient and stable contributors to the teacher-child relationship. Gender has been demonstrated to play a role in that relationship, with teachers reporting more conflict and less closeness with boys than girls (Rudasill, 2011; Rudasill, Reio, Stipanovic, \& Taylor, 2010; Rudasill \& Rimm-Kaufman, 2009). Evidence of this difference is found at school entry as teachers rate girls higher on ratings of closeness than they do boys. In fact, the gap in the rating of closeness between 
girls and boys seems to widen over the course of the later school years (Jerome et al., 2009).

Disadvantages in the teacher-child relationship have been identified for ethnic minority and low SES children, who tend to be rated by their teachers with less close and more conflictual relationships (Ladd et al., 1999; Wyrick \& Rudasill, 2009). Jerome et al., (2009) studied this situation longitudinally and found that black children received higher ratings of conflict at school entry than did children of different racial descent. During the seven years of study, the gap between teacher ratings of conflict for black children and white children became greater and regressed only slightly at the beginning of middle school - even when researchers controlled for a variety of influential variables (i.e., academic achievement, gender, behavioral problems, maternal sensitivity, maternal education, and time spent in non-maternal childcare). Of these individual characteristics, gender and race were found to have lasting and increasing association with teachers' perceptions of closeness and conflict over time (Jerome et al., 2009).

In addition to gender, the child's level of shyness or effortful control is an important factor in the teacher-child relationship (Rudasill, 2011; Rudasill et al., 2010; Rudasill \& Rimm-Kaufman, 2009). Rudasill and Rimm-Kaufman (2009) examined teacher-child relationship quality through teacher-and child-initiated interactions in first grade, in relation to ratings of these children's shyness and effortful control in preschool. Results indicate that shyness and effortful control were linked to later teacher-child relationships through the mechanism of teacher-child interactions - specifically, childinitiated interactions. Children with low shyness ratings in preschool tended to have higher ratings of both conflict and closeness with teachers in first grade, while children 
with low effortful control had higher ratings of conflict and those with high effortful control had higher ratings of closeness. Children who were rated low in shyness "were more likely to initiate interactions with teachers, and more child-initiated interactions were related to more teacher-child closeness." (Rudasill, 2011, p. 147). Furthermore, Rudasill and Rimm-Kaufman (2009) found evidence of a bi-directional relationship between teacher- and child-initiated interactions in that more child-initiated interactions were related to more teacher-initiated interactions, while more teacher-initiated interactions were related to fewer child-initiated interactions. Clearly, children's effortful control is a contributing factor to the quantity, and likely the quality, of interactions between teachers and children in their classroom.

Rudasill (2011) extended this line of research by introducing teacher- and childinitiated interactions in third grade to the model. The findings from this latest work support the idea that children's early relationships establish a foundation for later relationships. For example, early ratings of a child's shyness and effortful control are related to the frequency of interactions they initiate with their first (Rudasill \& RimmKaufman, 2009) and third (Rudasill, 2011) grade teachers. Teachers' perceptions of the teacher-child relationship and the number of teacher-initiated interactions in first grade predicted teachers' perceptions of the teacher-child relationship and the number of teacher-initiated interactions in third grade. Throughout both studies, gender remained a stable predictor of relationship quality and level of teacher- and child- initiated interactions.

These findings lend themselves to this dissertation in that Rudasill (2011) found that teacher-child relationship quality in first grade and the number of teacher- and child- 
initiated interactions in third grade worked to mediate the associations between children's characteristics and teacher-child relationship quality in third grade. In essence, this finding fortifies the importance of intervening in destructive relationships early, and provides hope for future relationships if the intervention is successful. Children who demonstrate externalizing behaviors and thus are at risk for the development of maladaptive behaviors may be more sensitive to the quality of teacher-child relationships (e.g., Burchinal, Peisner-Feinberg, Pianta, \& Howes, 2002; Hughes, Cavell, \& Jackson, 1999; Meehan, Hughes, \& Cavell, 2003; Silver, Measelle, Armstrong, \& Essex, 2005; 2010).

Given that effortful control is associated with children's externalizing behavior problems (Olson, Sameroff, Kerr, Lopez, \& Wellman, 2005), the work of Rudasill and colleagues provides valuable explanations regarding the way in which teachers interact with and form relationships with children with lower effortful control. Along with evidence indicating that positive teacher-child relationships work to decrease aggression in children (Meehan et al., 2003), the aforementioned differential effect further highlights the protective potential of positive teacher-child relationships and the necessity of intervening early in the development of coercive relationships.

In summary, coercive cycles of interaction (Patterson et al., 1992) are theorized to be the result of transactional influences between the environment, parents and teachers and individual characteristics. Given the relative stability of individuals within the home environment, children are likely to encounter repeated cycles of transactions with the same individuals that reinforce patterns of behavior over time. Each transaction shapes and reinforces children's behavioral orientation, increasing the likelihood that similar 
processes will be utilized within and between future environments and individuals (i.e., school and teachers). From this perspective we can trace the influences of coercive parent-child relationships from home to school, where a child learns to use coercion in interactions with parents/caregivers and these patterns are subsequently replicated in the school setting with teachers (Gunter \& Coutinho, 1997; Gunter, Denny, Jack, Shores, \& Nelson, 1993; Gunter et al., 1994; see also Jerome et al., 2009; Myers \& Pianta, 2008).

Thus, teacher-child relationships may be influenced by the relational experiences children bring from their homes to school, and as the child matriculates through school, remain stable. Teacher-child relationships can be promotive factors in children's school adjustment, and may be more important as protective factors for children with serious school adjustment and behavior problems. As teacher-child relationships have proven to be important protective factors in the development of children's social, emotional and academic functioning (Baker, 2006; Birch \& Ladd, 1996; 1997; Ladd et al., 1999; Ladd \& Burgess, 1999), it is logical to measure these relationships during interventions that work to promote children's positive social behavior. Particularly as Hamre and Pianta (2001) suggest, teacher-child relationships are salient in the early school years and may better predict children's subsequent school adaptation than measures of more general indicators of competence (e.g., behavioral problems, attention, social skills, and academic challenges).

Altering the trajectory of teacher-child relationships for children whose relational patterns are dominated by coercive transactions may be possible, given adequate resources in schools, appropriately motivated teachers, and empirically supported practices / interventions. In the next section, a review of the literature base related to the 
application of Motivational Interviewing in education will be presented. Beginning with an introduction to MI (Miller \& Rollnick, 2002), followed by a review of the various interventions that have informed the development of the FSCCU, which is the focus of this dissertation.

\section{Interventions}

Often, children whose school career involves serious school adjustment and behavior problems suffer long-term academic disadvantages and developmental delays (See Adelman \& Taylor, 2007; Burns \& Hoagwood, 2002; Kutash, Duchnowski, \& Lynn, 2006). Unfortunately, empirically validated and comprehensive school interventions for these children are few, especially for those children who demonstrate the most serious problems. Stimulated by these debilitating trends in the social, psychological, and academic development of children with serious school adjustment and behavior problems, the search for empirically validated interventions is on the rise (Walker, Golly, Mclane, \& Kimmich, 2005). As previously mentioned, authors of two important scholarly reviews took up this search (See Greenberg et al., 1999; Leff et al., 2001) and provided information regarding the characteristics of effective interventions. Across both reviews, effective interventions (a) engage multiple intervention agents (e.g., parents and

teachers); (b) apply for at least one school year; (c) use multiple components (e.g., training for parents and teachers, direct intervention with the child); and (d) include multiple settings. Additionally, Masten and Gewirtz (2006) remind us of the importance of an ecological perspective when considering the effectiveness of interventions:

From a resilience framework perspective, interventions must not only be conceptualized in terms of both positive and negative outcomes, but they must 
also be developmentally and ecologically valid, taking into account the multiple, interrelated, bi-directional influences on a child and family over time. As noted earlier, it is increasingly recognized that, particularly for young children facing cumulative and/or chronic risks, interventions need to be multi-level, individually tailored in intensity, targeting multiple domains of competence, and of sufficient length to promote lasting change (Farran, 2000; Shonkoff \& Meisels, 2000;

Shonkoff \& Phillips, 2000). These interventions may aim to reduce risk, alter vulnerability, and promote resilience by adding assets, reducing risk in a child's life, or by changing the moderators of risk (such as social competence, selfregulation, attachment, etc.) to enhance protections for children. Many interventions, particularly comprehensive models that target multiple domains of development, utilize two or more of the above strategies. (p. 35)

Reid (1993) has argued that the involvement of three social agents (i.e., parents, teachers, and peers) is necessary to effectively intervene in the lives of children with serious school adjustment and behavior challenges. The coordinated involvement of primary caregivers, teachers, and peers is a key feature of the many of the interventions that have shaped this dissertation. These interventions incorporate the values of the ecological perspective as noted by Masten and Gewirtz (2006), and the characteristics of effective interventions as noted in scholarly reviews (Greenberg et al., 1999; Leff et al., 2001). Next, the interventions that have shaped the current proposal are reviewed in detail. This section of Chapter two provides a review of the following interventions: (a) a counseling technique known as Motivational Interviewing (Miller \& Rollnick, 2002); (b) the Parent Motivation Inventory (Nock \& Kazdin, 2005); (c) the Ecological Approach to Family Interventions 
and Treatment (Dishion and Stormshak, 2007); (d) the Classroom Check-up (Reinke et al., 2008), a professional development approach for teachers; and (e) the First Step to Success early intervention program (Walker, 1998) including enhancements to the original program (Frey, et al., 2011). This section ends with a brief review of the importance of the concepts of social validity and treatment integrity.

Motivational Interviewing. Motivational Interviewing (MI) is defined as "a client-centered, directive method for enhancing intrinsic motivation to change by exploring and resolving ambivalence" (Miller \& Rollnick, 2002, p.25). MI is founded on the belief that how one interacts with people has significant effects upon intrinsic motivation that leads to better change. The approach builds upon non-directive approaches developed by Carl Rogers (1959), and his theory regarding the critical counselor skills necessary to facilitate change (Frey et al., 2011).

Miller and Rollnick (2002) describe two phases of MI: Phase 1, pre-commitment, in which ambivalence (in regard to target behaviors) is resolved; and a Phase 2, postcommitment, in which commitment is strengthened and intrinsic motivation for change is activated to drive a collaborative change-planning process. Miller and Rollnick adapt client centered therapy by adding a "spirit" or MI environment for change, and four motivational counseling principles that are skillfully combined to direct a client towards change (Miller \& Rollnick, 2002). Client-centered therapy consists of open-ended questions, affirmation, reflections of empathy, and summaries (OARS). This is often combined with the three underlying constructs of evocation, collaboration, and autonomy, which are referred to as the spirit of MI. Evocation embodies the counselor's active elicitation of the client's personal reasons for change (desire, ability, reasons, needs and 
commitment to change [DARNC]). Collaboration is exemplified when the client takes a leading role in the dialogue and when the nature of the interaction is substantially influenced by the client's ideas. Autonomy is pervasive in the MI process, and is exemplified when the client's decisions guide the interactions. In addition to the spirit of MI, four counseling principles embody the techniques and strategies used. The counselor should express empathy, develop discrepancy, roll with resistance, and support selfefficacy (Miller \& Rollnick, 2002). These principles are utilized to build relationships, increase and define importance, manage resistance to change, and increase a client's confidence for change.

Frey et al., (2011) provide insight into the promise of MI in the perspective of school mental health. They provide a review of current research regarding the impact of MI across a variety of mental health and health and wellness domains, suggesting that the use of MI is efficacious in encouraging adult commitment to behavior change. Numerous reviews suggest that the use of $\mathrm{MI}$ in many cases produced gains that were maintained after the intervention ended and sustained over time (Dunn, DeRoo, \& Rivara, 2001; Miller \& Rollnick, 2002; Noonan \& Moyers, 1997). These reviews further indicate that even in abbreviated formats when adapted for various applications, the use of MI holds promise for motivating change. Similar findings come from meta-analytical reviews of MI, which provides an aggregated view of its effectiveness (See Burke, Arkowitz \& Menchola, 2003; Hettema, Steele, \& Miller, 2005; Vasilaki, Hosier, \& Cox, 2006). Recently, Lundahl, Tollefson, Kunz, Brownell, and Burke (2010) demonstrated the beneficial effect of MI across a wide variety of problem behaviors. 
More relevant to this proposal, the use of MI preceding more standard interventions (typically for substance abuse) has been shown to increase the length of time a participant stayed in treatment, the effort a participant put forth, and adherence to intervention protocols. More positive outcomes were measured for subjects who received MI than for those who did not (Aubrey, 1998; Bien, Miller, \& Boroughs, 1993; Brown \& Miller, 1993; Saunders, Wilkinson, \& Phillips, 1995).

These are promising findings as implementation challenges (i.e., fidelity) are inherently difficult within the field of education itself (Fixsen, Naoom, Blasé, Freidman, \& Wallace, 2005). It is especially promising for teachers working with children who demonstrate developing school adjustment and behavior problems. As suggested by Maag (2001), many negatively reinforcing behavior management techniques (harsh discipline, removing children from the classroom, advocating suspensions) are reinforcing to the teacher, because they are effective in the short term. Frey, et al. (2011) suggest “... it is easy to see why school professionals may resist evidence-based practices, which are typically proactive and require changes in teacher behavior or the environment, which implies the problem does not reside within the child, but at the very least is shared (i.e., transactional) between the child and the adults who control the child's environment" (p. 19). With this reasoning, Frey and his colleagues further suggest that MI may be employed to increase the fidelity with which schools implement evidence-based interventions.

Although the use of MI in educational settings to address school adjustment problems is limited, there is a growing literature base demonstrating its efficacy in addressing the motivation of parents, teachers, and students across a variety of issues 
related to school mental health services. To this end, Nock and colleagues, Dishion, Stormshak and colleagues, Reinke and colleagues, and Walker and colleagues have initiated promising lines of research on Adaptations to Motivational Interviewing (AMIs), which are defined by the use of non-Motivational Interviewing techniques in combination with those more commonly accepted in the field of MI to address the needs of a particular population (Burke, Arkowitz, \& Menchola, 2003).

The Parent Motivation Inventory. In a series of articles, Nock and various associates (Nock \& Ferriter, 2005; Nock \& Kazdin, 2005; Nock \& Photos, 2006) developed and tested a conceptual framework to enhance parental participation in interventions designed for children (the Participation Enhancement Intervention; PEI; see Nock \& Kazdin, 2005), which led to the development of the Parent Motivation Inventory (PMI). Their premise for the development of the PMI was that treatment attendance and adherence to treatment plans are the most basic necessities for effective treatment delivery. In regard to the treatment of youth, this necessarily concerns the parent's motivation to provide for their child's attendance and to support adherence to treatment plans. Until the development and subsequent testing of the PMI, no tools existed to measure a parent's motivation for their children's treatment. The PEI used elements of MI to provide a very brief (5-15 minute) intervention targeting parent motivation at several points during their children's treatment process. Along with MI elements, the PEI included the distribution of specific information about the importance of attending treatments and staying on track with treatment plans, and helped parents develop plans for overcoming any barriers they faced in attendance and adhering to the prescribed treatment. Using the PMI to evaluate the effectiveness of the PEI, Nock and Kazdin 
(2005) found that increases in parent motivation predicted parents' ratings of fewer barriers to their participation in treatment and in turn greater treatment attendance. Furthermore, both parents and therapists reported greater adherence to treatment plans as a result of the PEI.

\section{The Ecological Approach to Family Interventions and Treatment (EcoFIT).}

Dishion and Stormshak (2007) have developed the EcoFIT model, which includes an assessment-driven feedback component delivered within the context of MI and has been applied within the context of schools (Dishion, Stormshak, \& Siler, 2010). Dishion and Stormshak (2007) effectively argue that the complex issues faced by families and their children cannot be approached nor ameliorated with typical interventions that focus effort narrowly (on singular members of the family or single issues). Rather, an ecological approach, which recognizes the importance of the individuals themselves (their interactions and the environment and culture in which they live) is necessary to effect change.

A hallmark component of the EcoFIT model, the Family Check-up, inspired by the Drinker's Check-up (Miller \& Sovereign, 1989), is designed to increase parenting behavior that promotes youth adjustment and competence through the use of MI. The results of a recent clinical trial provided preliminary findings for the efficacy of this intervention, as mothers in the intervention group showed increases in involvement in their child's behavior and their children showed corresponding decreases in conduct problems (see Shaw, Dishion, Supplee, \& Gardner, 2006). Dishion and Stormshak (2007) recommend that the Family Check-up program precede evidence-based interventions to increase parental compliance with treatment protocols and regimens. 
Lunkenheimer et al. (2008) conducted the first of a series of notable studies of the Family Check-up program in early childhood; results demonstrated the positive longitudinal effects of the program on very young children who were identified as at-risk for early conduct problems. Those children of low-income families, who were randomly assigned to the Family Check-up condition, demonstrated improvements in school readiness (i.e., inhibitory control and language development) through its effects on parents' provision of increased positive behavioral support. Further study of the program (see Gardner, et al., 2009) demonstrated the program's effectiveness for families "with very high levels of distress and disadvantage compared with those who were more advantaged..." (p. 550). These effects were not as strong in single-parent families.

The Classroom Check-up. The Classroom Check-up (Reinke et al., 2008) is designed to motivate teachers in the school setting to examine classroom management practices associated with school success and to develop a plan of action that focuses on improving management practices (specifically the teacher's use of praise and reprimands). It uses MI to leverage teachers' goals and values for their classrooms.

The Classroom Check-up (Reinke et al., 2008) utilizes performance feedback, which entails the provision of objective data-based information on the current performance of an individual with specified or targeted behaviors (a priori), and has been utilized to support teachers in numerous applications (see Sutherland et al., 2000 for a brief review). Commonly associated with performance feedback are frequent consultation meetings (Jones, Wickstrom, \& Friman, 1997; Noell, et al., 2005). Reinke, Lewis-Palmer, and Martin (2007) evaluated the effects of daily visual representations of objective databased information without frequent consultations on a sample of teachers $(n=3)$ whose 
targeted behavior was the increased use of behavior-specific praise. In this study, using multiple baseline design across three elementary classrooms, one focus child (identified by teacher as exhibiting problem behaviors) and one randomly selected peer (used as a comparison) per classroom were observed for problem behaviors. The teachers were observed for their use of general or behavior-specific praise.

Reinke provided the teachers with three thirty-minute consultation meetings, one prior to the use of the visual feedback and two during the course of the (approximately) 30 days of intervention. During the first consultation, the focus included the benefits of behavior-specific praise as well the methods used to interpret the visual display of the data (graphs that were created using Microsoft Excel). Subsequent consultations focused on the challenges of using behavior-specific praise and potential solutions to challenges related to the teacher's current situations. During these meetings no performance data was shared or discussed.

Baseline data revealed low and inconsistent use of behavior-specific praise for each of the three teachers, moderate to significant rates of disruptive behavior for the focus children, and low rates of disruptive behavior for peer comparisons. The low level of behavior-specific praise continued even after the first consultation meeting in which the benefits of this practice were discussed. The implementation of the visual data positively influenced the teachers' provision of behavior-specific praise, as steady increases in the frequency for both the focus child and the peer was evident. The increased use of behavior-specific praise seemed to have ameliorative effects on problem behaviors as the frequency of focus child disruptive behaviors fell (Reinke et al., 2007). Unfortunately, the motivational effects of the intervention were short-lived as the rate of 
behavior specific praise began to trend downward for at least two of the teachers, and was not sustained at similar rates after the use of visual feedback was discontinued (during a two-week follow-up observation).

Reinke et al., (2008) recently introduced a model of intervention similar to their previous work, which addresses the motivation of teachers to maintain the increased levels of behavior-specific praise. Based on the work of Miller and Rollnick (2002), and Dishion \& Stormshak (2007), the Classroom Check-up (Reinke et al., 2008) provides specific motivational enhancement strategies including individual feedback to teachers on observed classroom behaviors, identification of strengths, autonomy in the decision making process, guidance when requested, support for teacher self-efficacy, and a menu of options. Furthermore, the Classroom Check-up approach provides teachers with a visual feedback system based on observed behaviors (i.e. specific praise \& reprimands) as was demonstrated effective previously (Reinke et al., 2007). Results from this more recent study (Reinke et al., 2008), which utilized a multiple-baseline design across four teachers, indicated increased use of behavior-specific praise and reduced reprimands for all teachers. The study also documented decreases in classroom disruptive behavior for two of the classrooms. The authors noted the "decreases in classroom disruptions directly coincided with increased rates of praise" (p. 11). Differing from the results of Reinke's 2007 study on the use of visual performance feedback the addition of MI seemed to influence the sustainability of the positive findings. Follow-up data, collected on the behavioral changes for teachers one month following the end of the intervention, indicated that teachers had maintained higher rates of praise than were seen at baseline. In summary, the use of visual performance feedback and motivational 
interviewing, separately and as combined in the Classroom Check-up (Reinke et al., 2008) have been effective for increasing teachers' use of praise and reducing teachers' use of negative attention in classrooms with children exhibiting problem behaviors for a small sample of teachers under highly controlled intervention conditions. The classroom outcome observed in association with teachers' increased praise and decreased negative attention was decreased student disruptive behavior.

The Enhanced First Step to Success. Walker and his colleagues have begun taking the existing First Step to Success homeBase component (Walker, et al., 1998) and infusing it with the Family Check-up (Dishion \& Stormshak, 2007; Shaw, et al., 2006). An initial conceptualization of this intervention, the Enhanced First Step to Success (EFS), was recently been pilot-tested with nine families in Louisville, Kentucky, and is currently being revised. The revision includes infusing First Step CLASS component with the Classroom Check-up (Reinke, et al., 2008), and is the focus of this dissertation. The original First Step to Success Program, and the literature that supports it, is described next.

The First Step to Success. First Step to Success is an early intervention program designed for at-risk preschool and primary level, elementary school children who show clear signs of emerging externalizing behavior patterns (Walker, 1998). The First Step program was initially developed in 1992 and has been extensively evaluated using multiple designs, including single subject (Carter, \& Horner, 2007, 2009; Golly et al., 2000; Overton et al., 2002; Sprague, \& Perkins, 2009), longitudinal (Nelson et al., 2009; Walker et al., 1998), quasi-experimental (Diken, \& Rutherford, 2005; Golly, Stiller, \& Walker, 1998), and experimental (Walker et al., 2005; Walker et al., 2009). These 
evaluations have demonstrated strong, positive classroom effects across the majority of the at-risk preschool and primary level elementary school children with moderate or emerging behavior disorders that were treated. Significant among these effects are reductions in problem behaviors and increased social skills as rated by teachers, and increased academic engaged time as coded by trained observers. Many of these studies reported that children who participate in the program move to within normal ranges on measures of maladaptive and adaptive behavior.

Initial evaluations of the First Step program utilized two groups of kindergarteners ( $\mathrm{n}=24$ and 22 respectively) that participated in the program during two successive school years (Walker et al., 1998; Walker, Stiller, \& Golly, 1998). The randomized, waitlist, control-group design included a follow-up collection of data one year later, in order to evaluate for any enduring effects of the initial intervention. Powerful effects, including reduction of problem behaviors and increases in social skills and academic engaged time, were found immediately following the intervention. The average effect size across all dependent measures utilized in the study was $d=.86$. The authors were also surprised to find that these effects were relatively durable. The mean average scores for intervention effects remained stable when measured during the children's first grade year. Using ANOVA, the authors evaluated differences between the post-test and follow-up mean scores and found no significant differences across measures or groups (save for one group's mean difference for academic engaged time, which was surprisingly higher in grade one). Golly Stiller, and Walker (1998) replicated the study with a group of 20 kindergarteners and demonstrated similar results. No specific measures of fidelity were utilized for these studies; however, multiple methods (i.e., 
training, intervention logs, supervisory processes) led the authors to believe that the intervention was implemented with high levels of integrity.

A number of studies employing single subject-design methodology have produced similarly encouraging results. Specifically, Beard (1998) utilized a number of direct observational measures with six children who participated in the program, and found powerful reductions in problem classroom behaviors. Additionally, Golly et al. (2000) investigated the program effects for two sets of identical twins in kindergarten. Again, increases in academic engaged time and decreases in problem behaviors (five specific behaviors) resulted from the programs implementation. Lien-Thorne \& Kamps (2005) also explored the effects of the First Step program with three first and second grade children using a multiple baseline design across these participants and utilized additional reinforcement systems based on behavioral contingencies. Results indicated dramatic increases in children's academic engaged time and decreases in teacher ratings of problem behaviors. Russell, Carter, and Horner (2007) further extended research on the intervention, also within the context of a single subject design study, by adding a function-based evaluation to the First Step procedures with a child who did not respond to the typical First Step intervention. These authors revealed the flexibility of the intervention by demonstrating the addition of function-based adaptations could enable the child to complete the intervention process successfully.

Recently, the U.S. Department of Education reported on the effectiveness of the First Step to Success early intervention program in a What Works Clearinghouse Intervention Report (2012). This review's rigorous effectiveness standards found that the 
First Step program has positive effects on externalizing behavior, and potentially positive effects on social outcomes.

The First Step program has also been adapted to the developmental level of preschoolers and designed to fit within the context of early education settings (Walker et al., 2002). Adaptations to the program included intensified teacher training, extended First Step behavior coach support, frequent role-play with the focus child, and individualized rewards opportunities. The adapted version of the First Step program for preschoolers is currently the subject of an Institute of Educational Sciences efficacy trial. Pilot study began in seven classrooms in Kentucky and 10 classrooms in Oregon during the winter and spring of 2009, with promising results. During the 2009-2010 school year a pre-experimental pre- post-test design was employed with 42 families ( 24 Kentucky and 18 in Oregon). Half were assigned to an intervention condition and half were assigned to a comparison condition. While all students made some improvements in social skills and problem behaviors from baseline to post-test, children receiving the First Step intervention were more than twice as likely to demonstrate improvements on one or more teacher-reported measures (Frey, Seeley, Small, Feil, \& Walker, in press). Social validity measures indicate preschool teacher and parent satisfaction results were very high during the both the pilot and first years of the intervention.

Recently, Walker et al. (2009) completed the first large-scale efficacy study of the intervention $(n=200)$, demonstrating its applicability across a large urban school district. Although immediate results were similar to previous studies, the lasting impact of the program was clearly different than has previously been found. 
First through third grade students with externalizing behavioral problems from the Albuquerque, New Mexico public school system participated in this randomized controlled trial of the First Step to Success Early Intervention. Of the two hundred families who agreed to participate, 101 were randomly assigned to the intervention group, and 99 were assigned to the usual care group. Those in the intervention group took part in the First Step program, which lasted approximately three months (Walker et al., 2009), and consisted of three main components: universal screening, school component (CLASS), and a home component for parent training called homeBase (see Appendix A for complete summary of the intervention components). During the universal screening, student's problem behaviors, social skills, and academic abilities were assessed. These assessments were completed before the intervention for use as baseline data and eligibility criteria, as well as approximately three months later, as post-test data.

The measurement model included pre- and post- assessment across three domains: symptomotolgy, functional social impairment, and academic competence. Parents, teachers and trained observers acted as informants for these data, which included results from the Social Skills Rating System (SSRS; Gresham \& Elliott, 1990), the Systematic Screening for Behavioral Disorders (SSBD; Walker \& Severson, 1990), the Letter-Word Identification subtest of the Woodcock-Johnson III Diagnostic Reading Battery (WJ-III DRB; Woodcock, Mather, \& Schrank, 2004), an oral reading fluency test (Fuchs, 2003), and an observational measure of Academic Engaged Time from the SSBD. Follow-up data collection occurred approximately one year after the completion of the intervention. It included teacher-reported and parent-reported measures, academic assessments, and observational data. 
Walker and his collogues (2009) used multivariate models at posttest for each domain area, followed by univariate ANCOVA models (controlling for pretest results) to test for differences between intervention and control groups. Results for symptomotolgy, which included measurements of children's maladaptive and problem behaviors as reported by parents and teachers, were significant; children participating in the intervention group were found to have greater improvements in these areas than children in the usual care group. Effect sizes, ranged from $d=.62$ to .73 . The functional social impairment domain, which included measurement of children's adaptive behavior and social skills as reported by parents and teachers, were also significant; children participating in the intervention group were found to have greater improvements in these areas than children in the usual care group. Large effect sizes were found for teacher reports of children's adaptive behavior $(d=.82)$ and social skills $(d=.87)$. Moderate effect sizes were found for parent reports of children's social skills $(d=.54)$. Academic gains were found to be significant for children in the intervention group, as indicated by academic competence ( $d=.66$ as rated by teachers) and academic engaged time $(d=.44$ as rated by trained observers). No significant differences were obtained between the intervention and usual care groups for measures of children's oral reading fluency and vocabulary.

Practical significance of the intervention effects were evaluated by calculating a percentile rank improvement index for each of the school outcome measures within the three domains. Mean improvements of 25, 26, and 8 percentile points were achieved in the ratings of symptomotolgy, functional social impairment, and academic competence, respectively. Furthermore, the intervention was implemented with moderate to strong 
fidelity and integrity. Adherence to intervention protocols was adequate for coaches $(84 \%)$ and teachers ( $82 \%)$ across the four time points during which protocol adherence was observed. Working alliance was rated highly by both coaches (mean score $=4.5$ on a 5-point scale) and teachers (mean score $=4.7$ ) (Walker et al., 2009).

These results, especially those related to children's social functioning in the classroom, demonstrate the impact necessary to alter children's developmental course in the area of school adjustment and behavioral challenges (Walker et al., 2009). However, unlike previous research, these outcomes faded during follow-up investigations. One year after the initial intervention had ended, $91 \%$ of the original sample was contacted for completion of follow-up assessment materials. These data indicate that the strong effects demonstrated immediately following intervention phase were not sustained the following year (Walker et al., 2009). To address these issues, researchers developed a booster plan, for the following school year, to provide continuity for the First Step to Success student (First Step to Success Maintenance Plan Roadmap, October 2007).

In this experimental study, Walker et al. (2009) demonstrated the significant impact of the First Step program in reducing problem behaviors and increasing social skills and academic engaged time, as well as its limited positive impact on children's general academic ability across a large suburban school system. The study indicates that the intervention was provided with fidelity and integrity, and influenced behavior change in the focus children. Yet the challenges in sustaining program effects at one-year post intervention provides an opportunity to investigate additional variables that may function to support the children's behavior change over time. Specifically, the intervention's ability to influence behavior change in the teacher, in order to overcome the coercive 
cycles of interaction that tend to sustain children's challenging behavior, could be explored through measures of teacher behavior (i.e., teacher's use of praise and reprimands during the CLASS component).

Sprague and Perkins (2009), who set out to investigate the collateral benefits of the First Step program, provide a baseline measurement of teacher's use of praise and reprimands during the implementation of the First Step CLASS component. Their work represents the first measurement of these teacher behaviors during the implementation of the program, and provides the groundwork form which to address teacher behavior in this regard.

Utilizing a multiple baseline design across participants, Sprague and Perkins (2009) provide measures of teacher behavior change within four kindergarten classrooms implementing the First Step program. Focus children were identified to receive First Step based on universal screening procedures (the Early Screening Project; Walker, Severson, $\&$ Feil, 1994). These children represented the highest levels of externalizing behavior challenges in their respective classrooms. For each classroom, one additional child who displayed challenging behaviors (an alternate) and one child who represented typical behavior were also identified for observation. This study included the measures of children's problem behavior, academic engaged time, and social skills that are commonly found in previous First Step research. Additionally, measures of teacher-delivered positive and negative interactions with the focus child and the class as a whole were collected during brief (six minute) daily direct observations of the teacher. Additionally, teachers responded to a rating scale constructed to examine the teacher's perception of 
their own behavior (e.g., time spent focusing on inappropriate behavior, transitions, and positive/negative behaviors of the focus child and class).

As has been the case in the majority of research conducted on the First Step program over the past two decades, Sprague and Perkins (2009) found dramatic and immediate effects in the reduction of problem behaviors, increased social skills, and academic engaged time for the focus child. Collateral benefits in terms of challenging behavior were seen for the alternate children and typical peers. For these children, the combined average frequency of problem behaviors dropped at the onset of the intervention and maintained throughout. Significant increases in these children's academic engaged time were also found and maintained throughout the intervention.

More germane to the purposes of the current dissertation are what Sprague and Perkins (2009) called the collateral effects of the First Step CLASS intervention. Improvements were found in teachers' use of praise and reprimand and in their perceptions of their own behavior and the classroom ecology. The combined average frequency of positive interactions was 3.15 during baseline, 8.35 during the intervention phase, and 7.87 during the two-week follow-up phase. The combined average frequency of observed negative interactions across the four teachers was 7.65 during baseline, 3.38 during the intervention, and 2.45 at follow-up (Sprague \& Perkins, 2009). Additionally, "teacher ratings of their own behavior and perceptions regarding their effectiveness toward student behavior increased moderately from pre- to post-intervention" (p. 218), as did teachers' ratings of the classroom ecology. Unfortunately, these data were presented in aggregate, and could not be disaggregated for the focus children, the alternates, or the peers representing typical behavior patterns. Thus, the benefits of positive and negative 
teacher interactions are difficult to pinpoint. Was the teacher's shifting frequency of negative interactions to more positive interactions evenly distributed across these three students? Were the increases that were reported in aggregate due to increases in positive interactions with one or another of these three children? Moreover, what effect did these shifts in interaction patterns have for the relationship between the teacher and the focus child?

The First Step to Success program demonstrates strong, positive classroom effects across the majority of the at-risk preschool and primary level elementary school children with moderate or emerging behavior disorders that participated. Common findings for children who participate in the program are reductions in problem behaviors, increases in social skills, and improvements in academic engaged time. Early, small-scale studies resulted in sustained benefits of the program during follow-up investigations up to one full year after the completion of the program. However, these long-term benefits were not found in the follow-up study of a large-scale application of the program $(n=200)$ across a large urban school district (Walker et al., 2009). In this case, a maintenance program of continued teacher support and program booster sessions were necessary to maintain documented gains.

Evaluation of the program's collateral effects demonstrates benefits for children beyond the primary focus child who is selected for the intervention. In fact, peers in classrooms where First Step was implemented also reduced problem behavior, increased social skills and improved academic engaged time (Sprague \& Perkins, 2009).

Furthermore, teachers who were agents in the implementation of the First Step program demonstrated increased positive interactions and decreased negative interactions in the 
classroom overall as reported by combined (across individual students and the classroom as a whole) average frequencies. Unfortunately, the frequency of teacher's increased positive and decreased negative interactions as differentiated for each type of student (focus child or peers) is missing from this research. These factors are critical to the success of the First Step CLASS intervention, as classroom teachers' use of positive feedback increases the likelihood behavioral gains will be maintained while external reinforcers are faded, and works to reduce the negative attention for inappropriate behavior (often seen in coercive transactions), which inadvertently maintains the challenging behavior teachers seek to eliminate.

Also missing from research on the effectiveness of the First Step program are measures of the teacher-child relationship, and any changes to the relationship between a focus teacher and child that may be affected by the implementation of the program. The measurement of teacher-child relationships may be important, particularly in light of the demonstrated collateral effects of increased positive and decreased negative teacher child interactions, which seem to indicate the possibility of reduced coercive interactions typically seen between children with moderate or emerging behavior disorders and their teachers. Exploring any changes to the teacher-child relationship from baseline to postintervention, particularly as it relates to the child's response or lack of response to the intervention, may also shed light on the factors that challenge the sustainability of program effects that have been reported in large-scale applications of the intervention, and any improvements in teacher-child relations. The majority of research on the First Step program provides reasonable measures of fidelity of implementation and process integrity. However, there are no measures do not address the teacher's motivation to 
sustain newly learned practices in order to maintain the effects of the program for the long term. As a cross-disciplinary practice approach MI (Miller, 1985; Miller \& Rollnick, 2002) may provide an opportunity to address these issues. Enhancements to the First Step school component are designed so that changes in teacher and parent behavior as a result of the intervention procedures are maintained after the intervention period, thereby reducing the dependency on monitoring and booster sessions, and sustaining the impressive short term effects that the First Step to Success program consistently produces. In the enhanced version of the intervention, the home component has been completely revised. The CLASS component is implemented as articulated in the original version. However, This dissertation examined the enhanced version, which contains the FSCCU procedures based on Reinke et al.'s (2008) Classroom Check-up. Furthermore, this dissertation has examined the teacher-child relationship for the first time, and provides for the measurement and reporting of teacher behavior change. These enhancements work to expand the ecological reach of the First Step intervention, to more effectively alter the school ecology in supporting student achievement. The FSCCU is designed to increase teacher motivation to alter his or her own behavior - in support of the changing behavior of the focus child.

\section{Social Validity and Implementation Integrity}

Social validity includes the evaluation of interventions by those involved in its implementation and those who receive the intervention, to examine the social significance of intervention goals, the appropriateness of procedures, and the importance of outcomes (Frey, Park, Browne-Ferrigno, \& Korfhage, 2010; Schwartz \& Baer, 1991). The evaluation of social validity is important, as those who deliver and receive 
interventions may differ in their satisfaction with the purpose, process, and outcome of an intervention, which may influence acceptability, use, compliance, and effectiveness (Papalia-Berardi \& Hall, 2007). Lane \& Beebe-Frankenberger (2004) described social validity in this way:

It is important to assess social acceptability of intervention procedures...in order to ensure that all relevant parties (e.g., teachers, parents, and other interventionists) agree that the procedures are reasonable for the classroom, home, or wherever the intervention procedures take place...Namely, if an intervention is viewed as socially acceptable there is higher probability that it will be implemented with treatment integrity than if the intervention procedures were initially viewed to be unacceptable.

The measurement of social validity is an important variable in assessing the implementation of prevention and intervention programs (Hieneman \& Dunlap, 2000; Hieneman, Dunlap, \& Kincaid, 2005). Carr, Austin, Britton, Kellum, and Bailey (1999) reviewed articles from the Journal of Applied Behavior Analysis and found that less than $13 \%$ of articles reported social validity outcomes. Conroy, Dunlap, Clarke, and Alter (2005) suggested that researchers include social validity measures into their methodologies, after a review of positive behavioral intervention research with young behaviorally challenged children found that only $26 \%$ of studies conducted between 1984 and 2003 reported these measures.

It may be possible that newly developed interventions are theoretically rigorous, but lack social validity in the eyes of its consumers. From this perspective, evaluating the social validity of newly developed interventions is especially important, given that the 
consumer's feedback may be critical in redefining the intervention.

Treatment integrity is the extent to which an intervention is delivered as intended. Thus, a comprehensive understanding of treatment integrity is imperative to infer an intervention's effectiveness (Sheridan et al., 2009), and therefore critical to improving the intervention's outcomes.

Despite its importance in understanding and demonstrating effectiveness, treatment integrity in the field of education has been largely overlooked (Sanetti, Hagermoser, \& Kratochwill, 2009) or "assumed rather than assessed and empirically demonstrated" (Gresham, 1989, p. 47). Fixsen et al.'s (2005) classic synthesis of implementation research makes clear that while the science related to developing and identifying Evidenced Based Practices (EPB) is improving, the science related to treatment integrity, or implementing EBPs with fidelity so they produce the desired effects, is greatly lacking. A number of experts within the field of education have echoed this sentiment. For example, Sanetti, Hagermoser, and Kratochwill (2009) state, "without question, there is a gap between the methodological importance of ensuring treatment integrity and the available empirical support for intervention strategies to promote treatment integrity" (p. 451). These authors go on to state, "it is essential that school professionals have multiple strategies to promote high levels of treatment integrity." (p.453).

The enhancements to the First Step to Success intervention are premised on the idea that $\mathrm{MI}$ is a particularly promising approach for promoting treatment integrity, as it utilizes lessons learned from the fields of substance abuse and prevention sciences. Thus, an appropriate beginning point in the measurement of implementation integrity for MI as 
used in psycho-educational applications, would be the standards of proficiency utilized in these fields. As described in the following chapters, the quality with which MI is implemented in this study will be measured against the proficiency standards from the fields of substance abuse and prevention sciences - where MI originated.

Motivational Interviewing is a promising yet relatively untapped approach to enhancing the development and implementation of effective school-based interventions. This review has introduced the spirit and principles of MI and summarized literature related to the adaptations and effectiveness of MI in educational settings to create or improve existing interventions, or simply to increase the extent to which those known to be effective are implemented with fidelity. This review has highlighted the possibilities of increasing teacher involvement in interventions designed for children with serious behavior challenges with relatively brief intervention, and increasing the fidelity of interventions that depend largely on changes in teacher behavior. Based on initial attempts to use MI in the field of education with parents and teachers, the outlook for its use with teachers of children with severe behavior challenges is promising.

\section{Summary}

The First Step to Success early intervention program seeks to ameliorate the destructive effects of serious behavior problems that children with early developing school adjustment challenges often exhibit. As an ecological intervention, the program works in tandem with the focus child, the student's parents, and the classroom teacher in order to influence the transactional nature of these relationships. The First Step classroom component intervenes in the teacher-child relationship such that maladaptive behavior competencies (e.g., attention for negative behavior) are reduced, and adaptive 
ones (e.g., attention for positive behavior) enhanced - disrupting the coercive classroom transactions that often follow these children from home to school. The First Step to Success early intervention program has been extensively evaluated using multiple designs (see Walker et al., 2005). These evaluations demonstrate strong, positive classroom effects across the majority of the at-risk preschool and primary level elementary school children with moderate or emerging behavior disorders that participated. Significant among these effects are, reductions in problem behaviors, increased social skills, increased academic engaged time (Walker et al., 2009), and increased use of praise by teachers (Sprague \& Perkins, 2009).

This research also indicates that the original First Step to Success intervention (Walker, 1998; Walker et al., 1997) is not sufficient to substantially decrease problem behavior or to increase adaptive behavior of the most severely disordered students (Walker et al., 2009). In addition, contrary to previous research findings, when the intervention was applied in a large-scale study, challenges in sustaining program effects were found during a one-year follow-up. Data collection related to rates of positive versus negative feedback across coach, teacher, and maintenance phases of the intervention have been reported only once (Sprague \& Perkins, 2009). This data may elucidate the teacher's level of motivation to sustain newly learned practices in order to maintain the effects of the program for the long term. As well, measurement of the intervention's effect on the teacher-child relationship, as relational differences may account for the differing outcomes for students with serious school adjustment and behavior problems could be enlightening. Taken together, these two key elements may have compromised the researcher's ability to pinpoint issues leading to interventions 
diminished effects. The FSCCU enhancement of the First Step to Success early intervention program was developed to address these issues.

To investigate the feasibility and acceptability of the FSCCU, 12 teachers, three behavior coaches, and ten national advisory panel members participated in an open multiple-case-study research design, to (a) determine if the intervention was implemented as intended (implementation fidelity); (b) understand how key stakeholders received the intervention (social validity); (c) better understand the functioning of the intervention on potential mediators, outcome variables, and moderating variables. The following research questions were investigated.

\section{Research Questions.}

1. To what extent is the FSCCU implemented with fidelity (adherence \& quality)?

2. To what extent and under what circumstances do teachers and coaches perceive the FSCCU as a socially valid intervention?

3. To what extent and under what circumstances is the FSCCU functioning as intended? 


\section{CHAPTER III}

\section{METHODOLOGY}

The purpose of this study (which exists within the larger IES grant - Enhanced First Step to Success) is to develop measurement protocols, training materials and implementation procedures infusing the Classroom Check-up (Reinke et al., 2008) into the First Step CLASS component. These developmental goals will be informed by examining the feasibility and acceptability of the new intervention enhancement to the First Step to Success intervention referred to as First Step Classroom Check-up (FSCCU).

It is believed that fully developed training procedures will result in a socially valid (i.e. important and acceptable) intervention that can be implemented with fidelity, both with regard to procedural integrity and quality of MI skills. It is assumed that high levels of social validity and fidelity will result in an intervention that has the ability to be replicated, and a high likelihood of eventually being adopted in authentic educational settings. However, this does not ensure that the intervention will be effective. As an initial exploration of effectiveness, multiple cases will be examined to gain a better understanding of the intervention's functioning with respect to the logic model presented in Figure 2. It is believed that, if implemented well, the FSCCU will be effective in improving the coach-teacher and teacher-child relationship, teacher behavior, and the fidelity of the First Step CLASS component - all of which are likely mediating variables identified in the logic model. 
The feasibility analysis and the exploration of the variables from the logic model presented in Figure 2 will result in a fully developed manual and training materials. It will also reveal whether this enhancement promises to be effective. It is hoped that an exploration of the associations between these variables will result in a better understanding of the proposed logic model, and provide information to guide refinements to the measurement protocols, training materials, and implementation procedures of the FSCCU manualization effort. These refinements will improve the Enhanced First Step intervention procedures and inform an IES Goal 3 application to evaluate the Enhanced First Step intervention's efficacy. This study will address the following research questions.

1. To what extent is the FSCCU implemented with fidelity (adherence \& quality)?

2. To what extent and under what circumstances do teachers and coaches perceive the FSCCU as a socially valid intervention?

3. To what extent and under what circumstances is the FSCCU functioning as intended? 
Figure 2. First Step Classroom Check-up Logic Model.

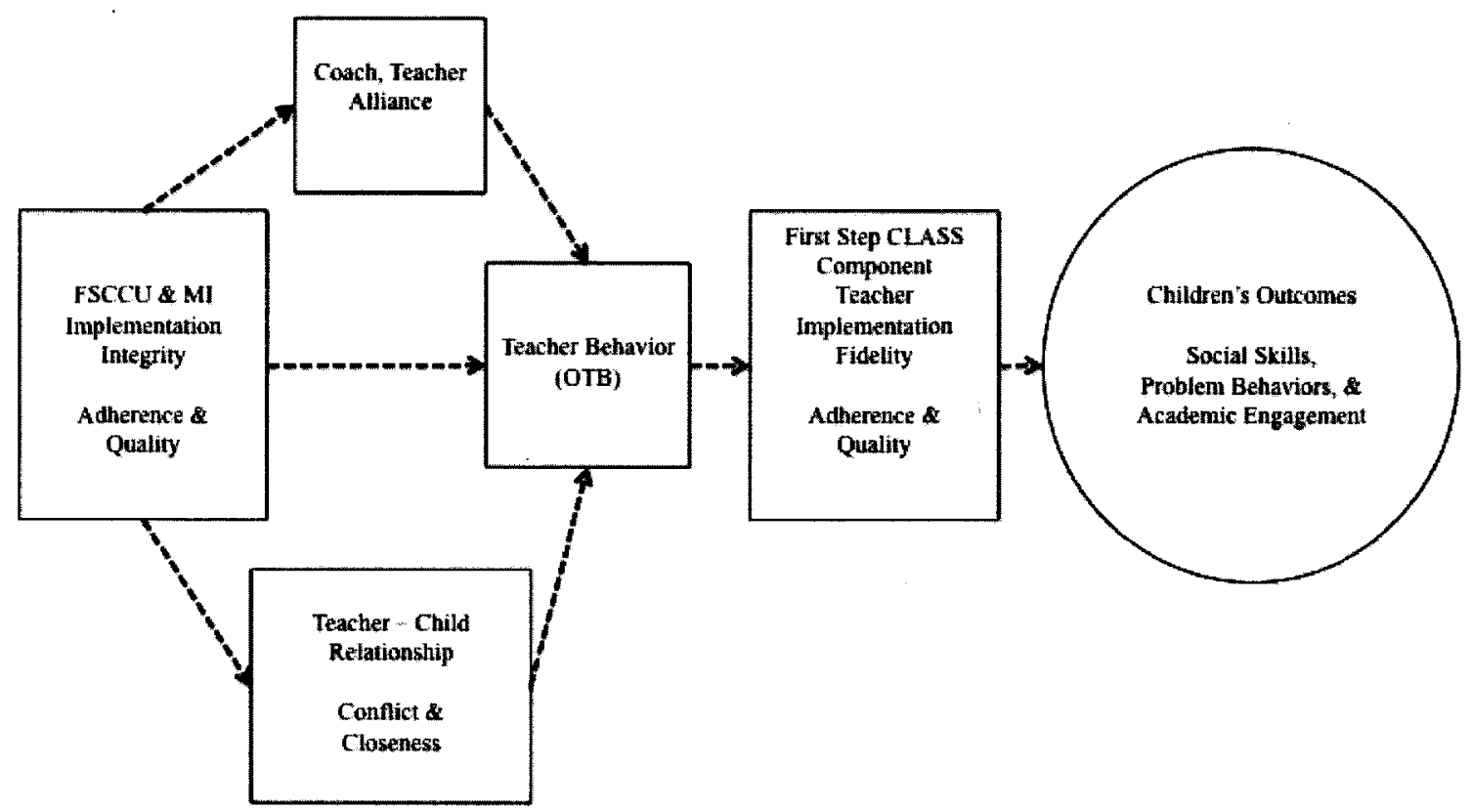

The following provides the details of the study setting, sample, and procedures, along with a description of each measure and procedure that is utilized. This chapter ends with a timeline of research events.

\section{Setting}

Focus schools, teachers, students and families were identified from within the Jefferson County Public School System (JCPS) in Louisville, Kentucky and the Greater Clark County School System in Jeffersonville, Indiana. The Jefferson County Public School System (JCPS) serves approximately 100,000 students in preschool through $12^{\text {th }}$ grade. The student population includes $54 \%$ who are white, $37 \%$ who are AfricanAmerican, and 5\% who are Hispanic. Sixty percent of the school district's students qualify for free or reduced lunch. Focus schools were selected by JCPS administration based upon levels of perceived need for support in the area of behavior and classroom management overall and the perceived likelihood of successful implementation. The 
Greater Clark County School System is one of the largest school corporations in the state of Indiana, with twelve elementary, four middle, three high, and two alternative schools. The district serves approximately 11,000 students in preschool through $12^{\text {th }}$ grade. The student population includes $71 \%$ who are white, $13 \%$ who are African American, and 6\% who are Hispanic. Sixty percent of the school district's students qualify for free or reduced lunch. One focus school was selected by Greater Clark County School administrators based on perceived need for support in the area of behavior and classroom management overall and the perceived likelihood of successful implementation.

\section{Sample}

The sample in this project was drawn from three schools in JCPS and one school from Greater Clark County Schools. One of the schools in JCPS, Waller Williams Environmental School, houses four self-contained primary level classrooms for children identified as Emotionally/Behaviorally Disturbed (EBD), and is the district's most restrictive placement option. The other three are typical elementary schools having at least one self-contained classroom at the primary level, and serving children identified as EBD in regular education classrooms and resource rooms.

For these schools, initial contact with teachers was made during brief school staff meetings with the research staff, who provided details about the procedures, risks, and benefits associated with participation. Following this, research staff met individually with teachers to provide in-depth information, and subsequently obtained their active consent to participate. Any assistant teachers expected to assume a substantial role in the implementation also consented to participate. 
Participating teachers sent a letter to the homes of parents, notifying them of the classroom-wide screening procedure (described below), the possibility of participation in the study, and an option to decline their child's participation in the universal screening. Teachers identified focus children after having had a minimum of 20 days of experience with them in their classrooms via the Systematic Screening for Behavior Disorders (SSBD), which utilizes a rank-ordering and rating procedure (Walker \& Severson, 1990). The SSBD multiple gating approach (Walker et al., 1988) is used to detect students in elementary grades who have an elevated risk for school behavior problems. The first two of three related screening stages, each with systematically increasing levels of scrutiny that assure the validity of results, were utilized to ensure that behavior impairment exists in the school setting. First, teachers nominate and rank-order children from their classrooms according to descriptions and examples of externalizing behavior profiles. Next, teachers rate nominated students' adaptive and maladaptive behaviors, and complete a Critical Events Checklist that provides information pertaining to significant risk factors. Students who exceed five critical events, and have an adaptive score of 30 or less and a maladaptive score of 35 or more, as a rule, have very serious school adjustment and/or behavior problems as indicated by analysis of archival school records and results of validation and follow-up studies (Walker \& Severson, 1990; Walker et al., 1990). Such students are strong candidates for specialized services/placements and/or identification as EBD.

Finally, parents of children who pass through the first two SSBD gates complete the externalizing subscale of the Child Behavior Checklist (CBCL; Achenbach, 1991), which is not associated with the SSBD, but was used as a third and final screening 
criteria for this study to ensure the existence of behavior impairment in the home setting. The externalizing subscale of the CBCL evaluates behaviors that tend to be directed outward such as, temper tantrums, physical aggression, and verbal bullying. The externalizing score is comprised of ratings of the child's rule-breaking and aggressive behaviors - at home. Families of children exceeding SSBD criteria were contacted in successive order, beginning with the family of the most severely impaired child and completed the CBCL during a phone interview. If the child exceeded the clinical or borderline threshold, the child and family were invited to participate. One child (and his or her family) per classroom who passed through both gates of the SSBD and had CBCL scores in the clinical or borderline range ( $\mathrm{T}$ Score $>63$ ) was invited to participate. Recruitment meetings at the school or in the home were scheduled for parents of these children to explore consent for participation in the study. This procedure was completed until there were no longer any eligible children in the classroom, or one child per classroom was enrolled. Focus children were verbally invited to assent to participate prior to each day's intervention activities, and in rare cases, depending on their age and developmental level, assented in writing.

\section{Procedure}

Provision of the intervention to teachers was preceded by the collection of all baseline data, three hours of teacher training in the First Step CLASS component procedures, and a parent meeting to explain the First Step to Success program in its entirety. The First Step to Success program was implemented with focus children and their families as described in Appendix A. Consistent with the purpose of this dissertation, 
the procedures specific to the FSCCU are presented next, followed by descriptions of the measures and analyses used to answer the research questions.

The First Step Classroom Check-up. The FSCCU is a process of teacher observation and interaction. The observation provides a limited view of the general positive or negative valence within the classroom, and a measure of the specific amount of praise and reprimand used by the teacher. As such, observations are completed during appropriate classroom activities in order to maximize the benefits of the First Step CLASS component (i.e., during academic engaged time). The interaction relies heavily on the use of Motivational Interviewing (Miller \& Rollnick, 2002), which embodies a client-centered approach of relationship building and seeks to resolve ambivalence between a teacher's ideals and current realities (especially pertaining to interactions with the focus child). Those unfamiliar with school-based applications of MI may benefit from referring to The Promise of Motivational Interviewing; Frey et al., 2011).

At this point in its development, the FSCCU consists of a series of four steps, as outlined in Table 1 (which also includes the tasks associated with each step). Although these four steps should occur in temporal order as listed, they may occur within the same meeting or occur in different meetings to allow for individual teacher needs and schedules. 
Table 1. The Four Steps of The First Step Classroom Check-up

\begin{tabular}{|c|c|}
\hline Steps & Tasks \\
\hline Information Gathering & $\begin{array}{l}\text { Complete two } 30 \text {-minute observations of teacher } \\
\text { behavior } \\
\text { Create a visual representation of the observational data }\end{array}$ \\
\hline $\begin{array}{l}\text { Getting to Know You } \\
\text { Interview }\end{array}$ & $\begin{array}{l}\text { Develop and assess a working alliance } \\
\text { Determine teacher's values and goals in relation to } \\
\text { teaching \& education } \\
\text { Listen for and elicit the teacher's experiences and } \\
\text { perceptions of school, teaching, and his or her use of } \\
\text { feedback (positive and negative) in the classroom } \\
\text { Facilitate teacher self-assessment of the five universal } \\
\text { principles }\end{array}$ \\
\hline $\begin{array}{l}\text { Data Review \& Goal } \\
\text { Setting }\end{array}$ & $\begin{array}{l}\text { Review observational data with the visual representation } \\
\text { Present menu of options (if needed) } \\
\text { Assess and manage resistance, amplify discrepancies, } \\
\text { cultivate importance, and boost the teacher's confidence } \\
\text { and feelings of self-efficacy for change } \\
\text { Develop plan of action } \\
\text { Identify goals and target dates for accomplishment }\end{array}$ \\
\hline $\begin{array}{l}\text { Maintenance } \\
\text { Observations \& } \\
\text { Feedback }\end{array}$ & $\begin{array}{l}\text { Complete four additional observations of teacher } \\
\text { behavior on or near specified days of the First Step } \\
\text { CLASS component (days } 6,10,16,20 \text { ) } \\
\text { Add maintenance observation data to the original graph } \\
\text { and provide it for the teacher } \\
\text { Celebrate progress, revisit menu of options, and manage } \\
\text { resistance to change as necessary }\end{array}$ \\
\hline
\end{tabular}

The Information Gathering process includes two 30-minute observations of five teacher behaviors. The frequency of praise (both behavior-specific and general) and reprimands are recorded across three agents: the focus child, any peer in the class, or the class as a whole. Data from these observations are used to create a graph of these behaviors, which is utilized during the Data Review and Goal Setting step. 
Coaches use the Getting to Know You step as an opportunity to develop and assess a working alliance between teacher and coach, using the spirit of MI to bolster the relationship. By exploring the teacher's experiences in and perceptions of public education, the coach works to identify a teacher's personal values and goals as an educator. These values and goals are crucial to the motivational interview, and assist in the amplification of any discrepancies that may exist between these ideals and their current use of praise and reprimands. The coach invites the teacher to complete the Classroom Expectations Checklist (Appendix C), offers the teacher a means to reflect on the principles of the First Step CLASS intervention, and asks the teacher to identify routines or expectations that could be enhanced by the utilization of these principles.

During the Data Review and Goal Setting step, the coach shares the results of observations, using the previously developed graph, and elicits the teacher's interpretation of the data. The coach utilizes a directive MI approach to assess and manage resistance, amplify discrepancies, cultivate importance, and boost the teacher's confidence and feelings of self-efficacy for change. During this process options are discussed, a plan of action is developed and formalized (typically in writing; Appendix D), and commitments to change are made. The goal is to improve the teacher's use of (i.e., unit rate ${ }^{2}$ ) behavior-specific praise, a critical component of the First Step CLASS component, while decreasing the teacher's use of negative attention to more appropriate ratios (if baseline levels are a concern).

Many options exist to help teachers facilitate a more positive climate through the use of increased positive behavior-specific praise. These options are self-selected, and

\footnotetext{
2 Unit rates of praise to reprimand are calculated by dividing total praise by total reprimand.
} 
self-monitored by the teacher with support from the coach when requested. Some of the more common self-selected options are listed in Table 2. In order to build a sense of ownership, reduce complexity and support self-efficacy, teachers are encouraged to create or utilize simple intervention strategies of their own design, if they do not select one from the menu of options provided.

Table 2: Self-selected Intervention Options for Teachers

\begin{tabular}{|c|c|}
\hline Intervention & Description \\
\hline Visual & $\begin{array}{l}\text { Use a handbill, posted at the back of the room or in a } \\
\text { conspicuous place, with a catch phrase or words of } \\
\text { encouragement reminding them to use positive behavior-specific } \\
\text { praise. }\end{array}$ \\
\hline $\begin{array}{l}\text { Goal for the } \\
\text { Day }\end{array}$ & $\begin{array}{l}\text { Create a goal for the day; specify a target level of positive } \\
\text { behavior-specific praise to reach (offer an incentive plan too!) }\end{array}$ \\
\hline $\begin{array}{l}\text { Class Review } \\
\text { of Expectations }\end{array}$ & $\begin{array}{l}\text { Teachers (and students) benefit from a group review of classroom } \\
\text { expectations. During this time expectations are explicitly taught } \\
\text { with examples and non-examples using the Green card. }\end{array}$ \\
\hline $\begin{array}{l}\text { Classroom } \\
\text { Attention } \\
\text { Signal }\end{array}$ & $\begin{array}{l}\text { Selection and use of an attention signal may be beneficial for } \\
\text { transitions, and as a request for student attention (i.e., a bell or } \\
\text { chime). }\end{array}$ \\
\hline $\begin{array}{l}\text { Double-up } \\
\text { Rule }\end{array}$ & $\begin{array}{l}\text { Teachers use the double-up rule as a mental reminder to provide } \\
\text { praise to an additional student each time they provide praise to } \\
\text { any one student. }\end{array}$ \\
\hline Paperclips & $\begin{array}{l}\text { Some teachers use paperclips placed on the First Steps to } \\
\text { Success program Green card to account for the optimal number } \\
\text { of praise statements that should be given during the intervention } \\
\text { period. After each praise statement is given, teachers remove a } \\
\text { clip, }\end{array}$ \\
\hline Wrist Bands & $\begin{array}{l}\text { Other teachers use "Wrist Bands" (colorful stretch rubber bands } \\
\text { in special shapes that can be purchased at any craft store). The } \\
\text { teacher wears the same number of bands on his or her arm as the } \\
\text { number of praise statements needed for that day. Every time the } \\
\text { teacher praises a student, they remove a "Wrist Band." }\end{array}$ \\
\hline
\end{tabular}


Four additional maintenance observations of teacher behavior follow the Data Review and Goals Setting interview. These make up the Maintenance Observations and Feedback step. Data from each additional observation are added to the original graph, with an indication of the expected amount of verbal praise associated with any goals previously determined, and are provided as feedback to the teacher without elaboration unless requested. This presentation of follow-up data allows the teacher to review progress and monitor the effectiveness of their self-selected interventions.

The process continues as necessary and as agreed upon by the teacher. Additional behaviors may be targeted, observed and discussed and the process ends with a celebration of accomplishments. Presented next is a description of the measures utilized for this study, followed by the analytic strategy to evaluate the research questions.

\section{Measures}

The measures utilized in this proposal are categorized as process and outcome instruments. Measures are presented as they are listed in the logic model (see Figure 2) from left to right and include the research question addressed by the measure. Each measure is followed by pertinent psychometric properties and a description of the point in time (i.e., baseline, post intervention) when the measure is collected.

Process Measures. The extent to which the FSCCU is implemented with fidelity will be addressed through the use of the Classroom Check-up Process Fidelity Checklist (Appendix E) and the Motivational Interviewing Treatment Integrity (MITI) code (see Appendix F). The First Step Classroom Check-up Process Fidelity Checklist addresses coach adherence to the intervention process, and a modified version of the MITI addresses quality of implementation. 
Classroom Check-up Process Fidelity Checklist. To measure adherence, a procedural checklist - the Classroom Check-up Process Fidelity Checklist as adapted from the work of Reinke, et al (2008) - will be utilized. Developed as a procedural checklist to guide interventionists through the FSCCU, it will be used to measure the percentage of tasks completed within and across all four steps: Information Gathering, Getting To know You, Data Review and Goal Setting, Maintenance Observations and Feedback. The Classroom Check-up Process Fidelity Checklist is based upon coach selfreport, and is completed throughout the intervention process.

The Motivational Interviewing Treatment Integrity (MITI) code. Quality of the intervention, particularly the application of MI, will be addressed using a modified version of MITI code. This measure has been adapted from the work of Moyers, Martin, Manuel, Miller, and Ernst (2007). The MITI code allows two investigators (both experts in $\mathrm{MI}$ ) to rate coaches' implementation of MI across five global domain areas (collaboration, autonomy and choice; directing clients towards change; understanding; reflection; and evocation), utilizing a five-point Likert-type scale ranging from strongly disagree to strongly agree. These five domain areas hereafter referred to as the global spirit, include what Miller and Rollnick (2002) define as the spirit of MI (evocation, collaboration, and autonomy). Additionally, coach utterances are assigned behavior codes, and a frequency count is recorded to account for the coach's MI skill and proficiency (Moyers, et al., 2007). Behavior codes include: open ended questions, close ended questions, simple reflections, complex reflections, MI adherent statements, MI nonadherent statements, and information giving. The MITI code demonstrates minimally adequate psychometric properties (Madson \& Campbell, 2006), with Moyers et al. (2005) 
reporting interclass correlations (ICC) to estimate the interrater reliability of the global ratings at .51 for empathy/understanding and .58 for the global spirit of MI. The intraclass correlations (ICC) for coach utterances ranged from .57 to .96 . Madson and Campbell (2006) indicate the MITI code to be a promising tool for research settings. Each FSCCU interview was coded, with reliability coding completed for $100 \%$ of all audio-recordings. The starting point for each recording was randomly chosen, and each tape was coded for 20 minutes. Coding was completed by the Clinical Training Institute.

Coach-Teacher Alliance Survey. The Coach-Teacher Alliance Survey (Appendix G) measures the coach-teacher relationship. The survey, a core measure disseminated by the National Behavior Research and Coordination Center (NBRCC), assesses perceived satisfaction with the this relationship as it pertains to implementation of the intervention. Walker et al. (2009) and Sumi et al. (in press) have utilized a 10-item version of this measure previously. In both studies the Coach-Teacher Alliance Survey was found to have strong internal consistency ( $\alpha=.94$, and .95 , respectively). The survey utilized for the current proposal consists of eight items on a five-point Likert-type scale ranging from never to always. Coaches and teachers answer the same questions, measuring the respondent's perception of shared goals, communication, trust, and effectiveness of the partnership with respect to implementation. Both teachers and coaches complete the survey as a post-intervention measure immediately following the completion of the First Step CLASS component.

Enhanced First Step CLASS Component Fidelity Checklist. The Enhanced First Step CLASS Component Fidelity Checklist (Appendix H) is the first of two measures utilized to gauge the fidelity of teacher implementation of the First Step CLASS 
component with the focus child. This 20 -item checklist measures procedural fidelity, or the extent to which the teacher adheres to guidelines for correctly implementing the CLASS intervention. First Step coaches complete the checklist for each of their assigned classrooms at two time points during the teacher phase of the First Step CLASS component. For each question the coach marks a response indicating whether the component was implemented (i.e., yes, no) and rates the quality of implementation on a five point Likert-type scale ranging from very poor to excellent.

Enhanced First Step to Success CLASS monitoring log. The Enhanced First Step to Success CLASS Monitoring Log (Appendix I) provides a second indicator (i.e., dosage) of the fidelity of the First Step CLASS component. The monitoring log was maintained on a daily basis by the coach and teacher. Included on this form are the intervention dates, total number of program days completed or recycled, point accumulations, rewards earned, and relevant notes. Additionally, information from this $\log$ will be utilized to arrange a chronology of intervention elements at the case level. Given these chronologies, the sequence of intervention elements can be analyzed across all cases and reviewed for program completion and possible barriers to implementation fidelity (e.g., prolonged absences).

Student-Teacher Relationship Scale. The short form of the Student-Teacher Relationship Scale (Pinata, 2001; STRS; Appendix J) is utilized to rate the teacher's perception of his or her relationship with the focus child. "The short form of the STRS is a self-report instrument comprised of 15 items rated on five-point Likert-type scale that assesses a teacher's perception of her relationship with a particular student" (Pinata \& Shulman, 2004, p. 450). The Student-Teacher Relationship Scale demonstrates strong 
internal consistency for two of the three subscales, Closeness and Conflict $(\alpha=.86$ and .92 respectively), and for the total score (Cronbach's alpha $=.89$ ). Test-retest reliability was adequate over a 4-week period with significant correlations $(p<.05)$ for Closeness, .88 , Conflict, .92 , and the total score, .89 . For this study, the classroom teacher completed only the Closeness and Conflict subscales, at baseline and post intervention.

Social validity. Coaches and teachers respond to questions designed to measure the overall importance and the acceptability of goals, procedures, and outcomes for the FSCCU, as related to their role in and satisfaction with the intervention. Of the 16 questions (regarding all aspects of the larger Enhanced First Step grant) answered by coaches, three questions pertain to the FSCCU and utilize response options along a five point Likert-type response continuum ranging from strongly agree to strongly disagree. Of the 23 social validity questions answered by teachers, 10 questions pertain to the FSCCU and utilize the same response continuum (Appendix K). These questions were adapted from a satisfaction measure developed by the Oregon Research Institute (Eugene, Oregon) in collaboration with SRI International (Menlo Park, California) for use in previous First Step Research (Walker et al., 2009; Sumi et al., in press). Results from these studies indicate the original survey possessed strong internal consistency $(\alpha=.92$, and .90 , respectively). Social validity measures were collected from teachers and coaches immediately following the completion of the intervention. These data were utilized to explore teachers' satisfaction with the intervention as a possible barrier to teacher behavior change and/or poor implementation fidelity.

Focus group interviews. In order to more fully examine coach and teacher perceptions of the social validity of the FSCCU and solicit recommendations for 
improvement, all participating teachers were invited to join in focus group interviews. The interviews were designed to reveal perceptions of the importance of goals, the acceptability of procedures, and the perceived effectiveness of the FSCCU. Focus group discussions were audio taped and transcribed using NVivo qualitative software. A standardized interview protocol was used for discussions and interviews (Appendix L). The interviews took place at post-intervention only.

Outcome Measures. Outcome measures are conceptualized in relation to the logic model. In terms of outcomes proximal to the FSCCU intervention, the Observation of Teacher Behavior serves to measure teacher behavior change after participation in the FSCCU intervention. Child outcomes are considered more distal, and will be evaluated by three measures - the Problem Behavior and Social Skills subscales of the Social Skills Improvement System rating scales (Gresham \& Elliott, 2008; SSiS-RS) and Academically Engaged Time from the Systematic Screening of Behavior Disorders (SSBD; Walker \& Severson, 1990). Case-level outcome results are provided (Appendix M).

Observation of Teacher Behavior (OTB). The recording procedures for this observation, found in Appendix N (along with an observation form), are designed for recording the nature of the social interactive behavior engaged in by the teacher, and is adapted from the observation protocol utilized by Reinke et al., (2008). During the observation coaches code the frequency of the teacher's use of praise and reprimands. Teacher behavior is coded for the focus child, any other peer in the classroom, or as directed at the class as a whole. Additional specificity of these behaviors is captured by coding praise or reprimands as either specific (labeling a specific behavior) or general 
(lacking the label of a specific behavior). The OTB was used to measure teacher behavior during instruction, with two 30-minute baseline observations occurring during the Information Gathering step of the FSCCU, and four 15-minute intervention observations occurring during the Maintenance Observations and Feedback step. As such, teacher's use of positive and negative feedback will be observed over the course of two full hours, one at baseline and one during the intervention.

Social Skills Improvement System rating scales. The SSiS-RS is the second edition of the Social Skills Rating System (Gresham \& Elliot, 1990; SSRS). It provides an excellent measure of peer-to-peer and teacher-related social skills as well as a measure of the teacher's perceived importance of rated social skills as they relate to successful school adjustment. The SSIS-RS is a series of rating scales completed by teachers for this study across two domains (social skills and problem behaviors). Common social skills are measured within the domains of communication, cooperation, assertion, responsibility, empathy, engagement, and self-control. The Problem Behavior subscales include the domains of externalizing, bullying, hyperactivity/inattention, internalizing, and autism spectrum. The national standardization sample of the SSiS-RS included 4,550 children aged 3-18. The SSiS-RS demonstrates appropriate levels of research integrity based on the widespread use and substantial body of scholarly research on the SSRS Social Skills Rating System (Gresham \& Elliot, 1990). The psychometric properties of the SSiS-RS have been compared with those of the SSRS (Gresham, Elliott, Cook, Vance, \& Kettler, 2010; Gresham, Elliott, Kettler, 2010; Gresham, Vance, Elliott, \& Cook, 2011). Results of this systematic comparison indicate that the SSiS-RS offers a broader conceptualization of important social behaviors and is psychometrically superior to its 
predecessor. Teacher perception of the focus child's social skills and problem behaviors were collected at baseline and post intervention.

Academic engaged time. Walker and Severson (1990) report that a measure of academic engaged time (AET) is an important indicator of a student's academic involvement and overall adjustment to teacher and classroom expectations. A child's AET can be observed as their level of attention to the teacher, material and task; appropriate movement throughout the classroom and in response to teacher; appropriate requests; appropriate interaction with peers and teachers; and ability to listen and follow directions. First Step coaches and trained staff observed the focus child's AET during three 20-minute observations at baseline and post-intervention. Only one observation was completed per day. During the AET observation, the total amount of time that a student exhibits behavior consistent with the SSBD definition of academic engagement is recorded using a stopwatch. The sum of these three values is divided by the sum of the total time for the three observation periods (typically 60 minutes) and multiplied by 100 to compute an average academic engaged time at baseline and post-intervention. Normative data for the SSBD-AET is provided for a sample of 1,300 first through sixth grade children from 16 school districts across six states (Walker et al., 1990). These tables, found in the SSBD manual, are arranged according to gender, grade level, and internalizing or externalizing behavior problems. Inter-observer agreement is calculated by dividing the smaller score of two observers by the larger score. Mean inter-observer agreement coefficients across several published studies have ranged from .95 (Walker et al., 1994) to .98 (Quinn, Mathur, \& Rutherford, 1995). 
The final section of Chapter 3 provides a summary of the proposed analytical procedures.

\section{Analyses}

A perspective of the overarching research design is provided as an introduction. This is followed by the proposed analyses, arranged by the research question for which the analysis is designed to address.

To address the proposed research questions, both quantitative and qualitative analytic methods were utilized for approximately four cases from the sample of subjects participating in the larger Enhanced First Step to Success Grant during the fall semester of 2010 , and 10 cases from the spring semester of 2011. An open multiple-case-study design was "used to compare cases that share an essential element and provide an opportunity to observe variation on one or more key variables" (Meyers et al., 2007, p. 101). Qualitative methods serve to inform the development of measurement protocols, training materials and implementation procedures infusing the Classroom Check-up (Reinke et al., 2008) into the First Step CLASS intervention during the iterative development cycle (See Hoagwood, 2001). Quantitative methods, namely a pretest and posttest design, are employed as an initial exploration of the effectiveness of the FSCCU. This is consistent with the logic model. As such, the design does not include a comparison or control group. While the design does not control for potential threats to validity, it does serve the purpose of this proposal: (a) determine if the intervention was implemented as intended; (b) understand how key stakeholders received the intervention; (c) better understand the functioning of the intervention on potential mediators, outcome variables, and moderating variables. 


\section{To what extent is the First Step Classroom Check-up implemented with}

fidelity? The extent to which coaches implemented the FSCCU with fidelity was evaluated across the aspects of adherence and quality. Adherence to the protocol (i.e., the four steps; see Table 1) was examined by calculating the percentage of processes completed within and across all tasks, as measured by the Classroom Check-up Process Fidelity Checklist. It is not known what level of procedural fidelity is required to generate favorable outcomes. A descriptive analysis will provide valuable information about the effectiveness of the training procedures, and establish a benchmark for subsequent studies. The author will utilize the Classroom Check-up Process Fidelity Checklist, again, with all audio recordings as a reliability measure to insure the accuracy of coach report.

Implementation quality will be addressed through the calculation and analysis of composite global ratings (collaboration, autonomy and choice; directing clients towards change; understanding; reflection; and evocation / 5), and behaviorally coded coach utterances from the MITI code. Both indices are analyzed individually by case and aggregated across First Step behavior coaches $(n=3)$. The quality with which MI is implemented by First Step coaches with subject teachers was investigated utilizing summary scores and related competency thresholds as suggested by Moyers et al., (2007). These authors have established summary scores to provide a more complete picture of MI proficiency. The scores include ratios of MI adherent utterances and non-adherent utterances, open/closed ended questions, simple/complex reflections, and reflections/questions. For example, complex reflections are a key client-centered counseling skill, and are used during the FSCCU to convey a deeper meaning or paint a more complex picture of teacher verbalizations. Simply reporting the number of complex 
reflections used by the coach has no meaning relative to the coach's use of reflections overall; however, reporting the percentage of complex reflections used by the coach in relation to the total number of reflections used by the coach provides an indicator of MI proficiency. Table 3 provides the title of each summary score calculated, the method of calculation, and threshold scores for beginning proficiency and competency as recommended in the MITI code. These thresholds have been developed with the context of a direct service delivery model and in a clinical setting. As previously mentioned, ratings have been modified for school-based application.

\section{Table 3. MITI Code Summary Score Thresholds}

\begin{tabular}{lll}
\hline $\begin{array}{l}\text { Summary Code and } \\
\text { (Means of Calculation) }\end{array}$ & $\begin{array}{l}\text { Beginning } \\
\text { Threshold }\end{array}$ & $\begin{array}{l}\text { Competency } \\
\text { Threshold }\end{array}$ \\
\hline $\begin{array}{l}\text { Global Spirit Rating } \\
\text { (Collaboration, autonomy and choice; directing clients }\end{array}$ & & \\
$\begin{array}{l}\text { towards change; understanding; reflection; } \\
\text { and evocation / 5) }\end{array}$ & $\begin{array}{l}\text { Average of } \\
\begin{array}{l}\text { Reflection-to-Question Ratio } \\
\text { (Total Reflections / Open Questions + Closed Questions) }\end{array}\end{array}$ & $\begin{array}{l}\text { Average of } \\
4\end{array}$ \\
$\begin{array}{l}\text { Percent Open Questions } \\
\text { (Open Questions / Open Questions + Closed Questions) }\end{array}$ & $50 \%$ & 2 \\
$\begin{array}{l}\text { Percent Complex Reflections } \\
\text { (Complex Reflections / Total Reflections }\end{array}$ & $40 \%$ & $50 \%$ \\
$\begin{array}{l}\text { Percent MI Adherent } \\
\text { (MI Adherent / MI Adherent + MI Non-adherent) }\end{array}$ & $90 \%$ & $100 \%$ \\
\hline
\end{tabular}

Results from the Classroom Check-up Process Fidelity Checklist provide a measure of intervention adherence, while results from the MITI code address implementation quality. Together these measures address whether the current intervention training and supervision provided for coaches resulted in high treatment integrity. Analysis of summary scores from the MITI code within cases provides indications of the overall quality with which the use of MI was implemented, and areas of weakness which may be reviewed across cases in order to identify (a) common barriers to 
MI implementation, and (b) needed modification to the intervention procedure's, and (c) areas to improve the MI training and supervision of First Step coaches. Since this is the first study examining MI fidelity within a school-based application, this descriptive analysis will inform benchmarks for subsequent studies.

To what extent and under what circumstances do teachers and coaches perceive The First Step Classroom Check-up as a socially valid intervention? The extent to which teachers and coaches perceive the FSCCU as a socially valid intervention was addressed through the use of questionnaires and focus group interviews.

Social validity. Descriptive statistics and teacher responses to items on the social validity questionnaire were examined at the item level and by generating composite scores for the 10 teacher satisfaction and 3 coach satisfaction survey items. Coach and teacher perceptions of the intervention's social validity are thought to be

- poor, if the composite average is 3 or below;

- good, if the composite average is 3-4;

- strong if the composite average is 4 or above.

Calculation of item level and composite scores provide preliminary data from which to explore differences and similarities between teacher and coach perceptions of the intervention's social validity and possible associations with child outcomes and change in teacher-child relationships. The analysis of coach scores follows in the same way.

Focus group interviews. Qualitative analyses of teacher and coach responses to focus group interview questions were conducted using the thematic framework of Braun $\&$ Clarke (2006) for the analysis, interpretation, and reporting of qualitative data. Specifically, a constructionist approach was utilized for interpreting the data in which 
codes were assigned to explicit statements made by participants during interviews. Often referred to as inductive thematic analysis, these data were coded without preconception or previously constructed codes (although a researcher is never truly void of preconception). Furthermore, these data were explored with a semantic approach identifying explicit or surface level meanings rather than at a latent level involving assumptions of the underlying meaning of individual remarks.

Focus group interviews were guided by a standardized interview protocol that was used for discussions and interviews to elicit teachers' commentaries on broad themes and their perceptions of the FSCCU intervention. These interviews are audio recorded, then systematically coded using the NVivo 9 qualitative analysis software package (QSR Nvivo 9 Software. Melbourne, Australia: Qualitative Solutions and Research Pty Ltd., 2007).

To what extent and under what circumstances is the First Step Classroom Check-up functioning as intended? Figure 2 presents the hypothesized path of influence for the variables associated with the FSCCU, from proximal processes to more distal ones. It is presented as a means of clarifying hypothesized influences amongst these variables. Beginning at the left of the figure, implementation integrity is thought to exert influence on the coach-teacher alliance. Both the integrity with which the intervention is implemented and the coach-teacher alliance are thought to influence teacher behavior. This chain of variables is thought to enhance the integrity with which the teacher implements the First Step CLASS component with the child (i.e., fidelity) and the child's subsequent responsiveness to the First Step CLASS component as defined by increases in social skills and academic engaged time, and decreases in problem behavior. 
In order to better understand the function of the FSCCU in relation to outcome variables (child outcomes and teacher behavior), potential mediators (coach-teacher alliance, CLASS component fidelity, social validity) and/or possible moderating variables (teacher-child relationship), a systematic case-by-case study of all process and outcome measures was conducted prior to the analysis of these variables across cases. Specifically, detailed case summaries were constructed and analyzed in the context of the logic model. Case chronologies were developed to explore the circumstances under which the implementation of the FSCCU was associated with changes in child outcomes. The case summaries and chronologies for each teacher-child pairing include all logic model elements, results, and any irregularities in the intervention sequence (e.g., prolonged absences, missing data).

Analyses of the function of the FSCCU across cases were completed for (a) components of the logic model individually and (b) as a composite including all cases and components of the full logic model. Both analyses (case level and across cases) are dependent on a classification system of the results for each element of the logic model (case-level), so as to discern any patterns of influence. In the following section, the analysis for each element of the logic model is presented, as are the details of the classification system. Table 4 represents each measure, the way in which it is typically scored and the classification of results used for the purposes of this study.

Coach-teacher Alliance. Coach-teacher alliances will be analyzed as an average of the eight alliance survey items answered separately by coaches and teachers. Coachteacher alliances are thought to be

- poor, if the composite average is 3 or below; 
- good, if the composite average is 3-4;

- strong, if the composite average is 4 or above.

The composite average coach-teacher alliance for the sample (sum of individual composite average scores / sample size) will constitute the analysis of coach-teacher alliances across cases. Individual averages and sample composite averages for the survey were examined in relation to measures of teacher behavior change. Assuming the logic model is functioning as intended, there should be positive associations between MI quality and coach-teacher relationship.

Teacher behavior. To investigate the circumstances under which the implementation of the FSCCU is associated with improvements in teachers' use of praise vs. reprimands, observational data will be visually inspected using simple histograms of data points at baseline and post-intervention (maintenance observations). Individual cases were classified as FSCCU Responders, based on increases in the total average ratio of praise to reprimands provided overall (to the focus child, any peer, and the class as a whole) by the teacher. Successful improvements in the frequency of teacher praise is defined as an increase in total average baseline unit rate of praise to reprimands to a minimum total average post-intervention unit rate of praise to reprimands of 2.9. (Frederickson \& Losada, 2005, see also Sprague \& Perkins, 2009). Thus, responders to the FSCCU are classified as those teachers whose ratio of praise to reprimand reached 2.9 to 1 , as a total average across the four maintenance observations of the intervention.

Baseline versus post-intervention ratios were also explored across each observation category (focus child, any peer, class as a whole). Individual teacher behavior change was classified as successful or unsuccessful based on these data points 
to explore associations with child outcomes, as well as associations with the teacher-child relationship. Data from the OTB was analyzed within cases and across the sample, and reliability coding was completed for $10 \%$ of all observations.

CLASS intervention fidelity. Data from the Enhanced First Step CLASS Fidelity Checklist (utilized twice during the CLASS intervention) evaluates (a) component adherence (yes/no), and (b) the quality of component implementation using a five-point Likert-type scale $(0=$ Very Poor, $.25=$ Poor, $.50=$ Okay, $.75=$ Good, $1.0=$ Excellent $)$. Teacher adherence scores were calculated as the proportion of procedures correctly implemented. The mean of the two teacher adherence scores were computed to estimate an overall teacher adherence score. Quality ratings for the teacher were calculated as the mean score from both ratings of implementation quality. Adherence proportions above $80 \%$ represent adequate adherence. Quality ratings of $.75-.90$ represent adequate levels of CLASS component implementation quality, while excellent ratings of adherence and quality meet or exceed $90 \%$ and .90 respectively.

As an additional indicator of fidelity (i.e., dosage) data from the First Step to Success CLASS Monitoring Log will be analyzed. Intervention dosage was calculated as the proportion of program days delivered by the coach and teacher (out of 30 possible), and compliance was calculated as the proportion of days when the focus child met the daily point criteria. This information will be presented within the case summaries and chronologies.

Teacher-child relationship. Individual cases were evaluated for improvements in the teacher-child relationship, utilizing the STRS (Pianta, 2001). Given the open case design and low sample size of the proposed study, inferential statistical methods alone 
were not realistic to provide the case level information from which to better understand the benefits, potency and impact of the intervention on individual subjects. As such, a combination of approaches will be utilized to better understand the teacher-child relationship and its place in the proposed logic model.

A repeated measures ANOVA (equivalent to a paired-t given that the variable is measured only at two time points) was calculated to provide an aggregate effect size (partial eta-square) assessing within-subject change from baseline to post-intervention across cases.

To provide case-level information, and to determine if the change in teacher-child relationship were statistically reliable, the Reliability Change Index (Jacobson \& Truax, 1991; RCI) was calculated. The RCI takes into account the test-retest reliability of the STRS, provides an indication of cases that have responded to the intervention, and “...provide[s] a precise method for classifying clients as "changed" or "unchanged" on the basis of clinical significance criteria" (Jacobson \& Truax, 1991, p. 13)." The RCI is calculated on an individual case basis. A significant $\mathrm{RCI}$ statistic $(>/=1.96)$ indicates a reliable change in the teacher's perception of their relationship with the focus child. Results from the individual analyses using the RCI statistic can then be aggregated to determine the percent of students that improved.

Based on the classification of teacher-child relationship change, all other process variables, including teacher-child relationship at baseline, will be examined to better understand any associations that might be inferred between these variables. Although any relationships found may be linear, it is possible that they serve to moderate outcomes. For example, it is possible that a very low perception of the teacher-child relationship at 
baseline reduces the power of the intervention to affect teachers' increased use of praise vs. reprimands or to improve the relationship at all.

Child Outcome. Changes in teacher's rating of the focus child's social skills and problem behaviors, and observed changes in the focus child's AET was examined. Reliable improvement in the focus child's social skills and problem behaviors were evaluated by calculating the RCI (Jacobson \& Truax, 1991) as described previously. The significance of change in AET was evaluated based on the normative data provided in the SSBD Implementation Manual (Walker \& Severson, 1990). Children were classified as Responders, Partial Responders, or Non-responders based on SSiS results:

- Responders: Children with an RCI change statistic of greater than or equal to 1.96 on teachers' ratings of social skills and less than or equal to -1.96 on teachers' ratings of problem behaviors. This definition also includes any improvement (absolute change in the correct direction) in AET, the significance of which will be measured by the SSBD AET normative data (Walker et al., 1990).

- Partial Responders: Children with an RCI change statistic greater than or equal to 1.96 on teachers' ratings of social skills or less than or equal to -1.96 on teacher's ratings of problem behaviors for either social skills or problems behaviors, and any improvement (absolute change in the correct direction) in AET. Alternatively, a partial responder may also include any improvement (absolute change in the correct direction) for two of three measures (social skills, problem behaviors, AET) that do not reach clinical significance.

- Non-responders: Children with an RCI change statistic less than 1.96 on 
teacher's ratings of social skills or greater than -1.96 on teacher's ratings of problems behaviors, and no change in AET or a reduction in AET.

Table 4. The First Step Classroom Check-up Analysis Classification System.

\begin{tabular}{|c|c|c|}
\hline Measure & Standard Scoring & Classification \\
\hline Social Validity & $\begin{array}{l}\text { 1=Strongly Disagree } \\
\text { 2=Disagree, } 3=\text { =No Opinion, } \\
4=\text { Agree, } 5=\text { Strongly Agree }\end{array}$ & $\begin{array}{l}\text { Poor; average } 3 \text { or below } \\
\text { Good; average } 3-4 \\
\text { Strong; average is } 4 \text { or above }\end{array}$ \\
\hline $\begin{array}{l}\text { Coach-teacher } \\
\text { Alliance } \\
\text { Survey }\end{array}$ & $\begin{array}{l}1=\text { never, } 2=\text { seldom, } \\
3=\text { sometimes, } 4=\text { often, } \\
5=\text { always }\end{array}$ & $\begin{array}{l}\text { Poor; average } 3 \text { or below } \\
\text { Good; average } 3-4 \\
\text { Strong; average is } 4 \text { or above }\end{array}$ \\
\hline $\begin{array}{l}\text { Teacher } \\
\text { Behavior } \\
\text { (OTB) }\end{array}$ & $\begin{array}{l}\text { Average ratio of observed } \\
\text { positive to negative feedback }\end{array}$ & $\begin{array}{l}\text { Responders; total average ratio of } \\
\text { observed positive to negative feedback } \\
\text { at or above } 2.9: 1 \text {. Non-responders; } \\
\text { total average ratio of observed positive } \\
\text { to negative feedback below } 2.9: 1\end{array}$ \\
\hline $\begin{array}{l}\text { CLASS } \\
\text { Intervention } \\
\text { Fidelity - } \\
\text { Adherence }\end{array}$ & $\begin{array}{l}1=\text { Yes } \\
0=\text { No }\end{array}$ & $\begin{array}{l}\text { Adequate; proportion of components } \\
\text { implemented above } 80 \% \\
\text { Excellent; above } 90 \%\end{array}$ \\
\hline $\begin{array}{l}\text { CLASS } \\
\text { Intervention } \\
\text { Fidelity - } \\
\text { Quality }\end{array}$ & $\begin{array}{l}0=\text { Very Poor, } .25= \\
\text { Poor, } .50=\text { Okay, } .75= \\
\text { Good, } 1.0=\text { Excellent }\end{array}$ & $\begin{array}{l}\text { Adequate; } .75-.90 \\
\text { Excellent; above } .90\end{array}$ \\
\hline $\begin{array}{l}\text { Teacher-child } \\
\text { Relationship }\end{array}$ & $\begin{array}{l}\text { Raw Scores and Percentile } \\
\text { Ranks }\end{array}$ & $\begin{array}{l}\text { Responders; RCI statistic }>/=1.96 \\
\text { Non-responders; RCI statistic }<1.96\end{array}$ \\
\hline $\begin{array}{l}\text { Child } \\
\text { Outcomes - } \\
\text { SSiS-RS } \\
\text { Social Skills \& } \\
\text { Problem } \\
\text { Behavior }\end{array}$ & $\begin{array}{l}\text { Raw Score, Standard Score, } \\
\text { and Percentile Ranks }\end{array}$ & $\begin{array}{l}\text { Responders; }>1.96 \text { or }-1.96 \text { for both } \\
\text { domains. Partial Responders; }>1.96 \text { or } \\
-1.96 \text { for one domain } \text { or any positive } \\
\text { change for both domains that did not } \\
\text { reach significance. Non-responders; }< \\
1.96 \text { or }>-1.96 \text { for both domains }\end{array}$ \\
\hline $\begin{array}{l}\text { Child } \\
\text { Outcomes - } \\
\text { AET }\end{array}$ & $\begin{array}{l}\text { Average Observed Academic } \\
\text { Engaged Time }\end{array}$ & $\begin{array}{l}\text { Responders; any positive change, with } \\
\text { significance measured by the SSBD } \\
\text { Partial Responders; any positive } \\
\text { change. Non-Responders; no positive } \\
\text { change or any decline }\end{array}$ \\
\hline
\end{tabular}




\section{Summary}

This chapter presents the logic model, methodology and proposed measures to investigate teacher motivation as an enhancement to the First Step to Success intervention for children with serious school adjustment and behavior problems. One purpose of this study, which utilizes existing data from a larger IES grant, is to develop measurement protocols, training materials and implementation procedures infusing the Classroom Check-up (Reinke et al., 2008) into the First Step CLASS intervention (Walker et al., 1997). To this end an iterative process (See Hoagwood, 2001) was utilized to develop and refine the new intervention. For the purposes of this proposal, the refinement will be based on feedback from (a) key participants (teachers, coaches, and national advisors); (b) child outcomes; and (c) analysis of fidelity, feasibility and usability measures. In general terms, this process is utilized to (a) determine if the intervention was implemented as intended; (b) understand how key stakeholders received the intervention; (c) better understand the functioning of the intervention on potential mediators, outcome variables, and moderating variables.

Within this chapter, a logic model is provided to clarify the hypothesized path of influence amongst the variables associated with the intervention process. Specific measures are proposed to capture intervention effects, along with quantitative and qualitative methods to compare and contrast these effects to the logic model presented in order to investigate the following research questions.

1. To what extent is the FSCCU implemented with fidelity (adherence \& quality)?

2. To what extent and under what circumstances do teachers and coaches perceive the FSCCU as a socially valid intervention? 
3. To what extent and under what circumstances is the FSCCU functioning as intended? 


\section{CHAPTER IV}

\section{RESULTS}

This chapter presents the results of the study. Prior to the analyses, data were entered in to SPSS and descriptive statistics were examined to assess entry errors. Herein, an analysis of the recruitment and screening results is provided, followed by a description of the study participants. Next, the extent to which First Step coaches implemented the FSCCU intervention with fidelity (research question 1) is addressed. Then, survey data and focus group interview data addressing the extent to which teachers and coaches perceive the FSCCU as socially valid is presented (research question 2). Finally, case level and aggregate results are presented to better understand the extent to which the FSCCU functioned as intended in relation to outcome variables (child outcomes and teacher behavior), potential mediators (coach-teacher alliance, CLASS component fidelity, social validity) and/or possible moderating variables (teacher-child relationship; research question 3).

\section{Recruitment and Screening}

To examine the process and outcomes associated with the FSCCU intervention, two elementary schools (Wilkerson \& Layne), one self-contained school for children with behavioral disorders (Waller Williams Environmental School) in JCPS, and one elementary school in Greater Clark County (Parkwood), were recruited in August 2010 to participate. Thirty-three kindergarten through third grade teachers were eligible for participation in the study. Nine teachers declined participation, either because they were 
uninterested or confident they did not have a child that would qualify. Twenty-four teachers completed the universal screening to identify potential children and families to participate- three teachers from Waller Williams Environmental School, nine teachers from Parkwood Elementary, seven teachers from Wilkerson Elementary, and five teachers from Layne Elementary.

Eighty-eight children from 24 classrooms were screened. After completion of the parental passive consent and universal screening processes, five children were rank ordered within classrooms by their teachers (SSBD stage 1). Next, the teacher completed the Critical Events Index and the Adaptive and Maladaptive Behavior Indices (Walker \& Severson, 1990; SSBD stage 2) on each of the top five ranked children in their class. The parents of children whose SSBD stage 2 scores qualified them as the top ranked child (i.e., most severe behavior challenges) in each class were contacted to complete the externalizing scale of the Child Behavior Checklist- Parent Report Form (Achenbach, 1991; CBCL). Any children found to be in the clinical or borderline range on the CBCL externalizing scale (stage 3) met full inclusion criteria for the study. Due to the extreme need for these services at Waller Williams, two children were selected within each of the three classrooms, with one child participating in wave 1 (fall) and one participating in wave 2 (spring) of the study. Table 5 summarizes the screening data for these children.

The multiple gating screening procedures utilized for this study succeeded in producing a sample of children whose school adjustment and behavior problems were extreme. Forty three of the eighty-eight children met stage 2 criteria. Our screening procedures were designed to identify the most challenging child in each classroom. Often, second and third ranked children from the same classroom were not approached for 
consent- if the parents of the first ranked child in that classroom provided consent. Of the 24 classroom teachers who were invited to participate in the study, $18(75 \%)$ had at least one child who met stage two criteria (43 children in total). Within these 18 classrooms 21 children ( 6 from the 3 Waller Williams classrooms) met the criteria at stage 3 and were consented to participate across Waves $1(\mathrm{~N}=9)$ and $2(\mathrm{~N}=12)$.

Table 5. School-level Summary of Screening Data.

\begin{tabular}{lllll}
\hline Measure & $\begin{array}{l}\text { Layne } \\
\left(n=18^{*}\right)\end{array}$ & $\begin{array}{l}\text { Parkwood } \\
\left(n=36^{*}\right)\end{array}$ & $\begin{array}{l}\text { Wilkerson } \\
\left(n=21^{*}\right)\end{array}$ & $\begin{array}{l}\text { Waller } \\
\left(n=13^{*}\right)\end{array}$ \\
\hline CEI $M(S D)$ & $3.9(2.5)$ & $3.1(2.7)$ & $4.0(3.2)$ & $8.8(4.5)$ \\
ABI $M(S D)$ & $39.3(11.5)$ & $34.9(11.0)$ & $38.5(7.0)$ & $39.9(8.5)$ \\
MBI $M(S D)$ & $31.4(7.1)$ & $31.5(8.0)$ & $26.6(7.5)$ & $29.6(8.6)$ \\
CBCL Externalizing (TS) & $71.7(10.2)$ & $72.0(7.0)$ & $70.0(10.3)$ & $72.4(7.3)$ \\
Met Stage 2 criteria $n(\%)$ & $6(33.3)$ & $13(38.2)$ & $7(33.3)$ & $11(84.6)$ \\
\hline
\end{tabular}

* Sample size varies by measure. CEI = Critical Events Index; ABI = Adaptive Behavior Index; $\mathrm{MBI}=$ Maladaptive Behavior Index; $\mathrm{CBCL}=$ Child Behavior Checklist; $\mathrm{TS}=T$ score.

Sample. Twelve of the $21(57 \%)$ children whose parents consented to participate were included in the current study. Two families (9.5\% within-year attrition) moved prior to having completed the intervention. Additionally, three of the children from Wave 1 of the intervention classrooms at Waller-Williams Elementary school were excluded as the coach assigned to that school had not yet completed the FSCCU training and reliability requirements. Two teachers withdrew from the study, and two teachers chose not to participate in the FSCCU intervention component.

As can be seen in Table 6 , the sample consisted primarily of boys $(83 \%)$ whose average age would place them in the first grade. Sixty-seven percent of the sample was Caucasian while the remaining 33\% were African American. Participating teachers were 
predominately Caucasian women $(92 \%)$ with graduate degrees $(84 \%)$; they were veteran teachers, with an average of 10.6 years of teaching experience. Next, research question 1; to what extent is the FSCCU implemented with fidelity (adherence \& quality), is addressed.

\section{Table 6. Sample Characteristics}

\begin{tabular}{llrrr}
\hline Variable & & $\begin{array}{r}\text { Coach } \\
(\mathrm{N}=3)\end{array}$ & $\begin{array}{r}\text { Child } \\
(\mathrm{N}=12)\end{array}$ & $\begin{array}{r}\text { Teacher } \\
(\mathrm{N}=12)\end{array}$ \\
\hline Age $M(S D)$ & & -- & $6.8(1.1)$ & -- \\
Gender $n(\%)$ & Female & $2(66)$ & $2(17)$ & $11(92)$ \\
& Male & $1(33)$ & $10(83)$ & $1(8)$ \\
Ethnicity $n(\%)$ & African-American & -- & $4(33)$ & $1(8)$ \\
& Caucasian & $3(100)$ & $8(67)$ & $10(83)$ \\
& Native American & -- & - & $1(8)$ \\
Education $n(\%)$ & Some College & -- & -- & -- \\
& Bachelor's degree & -- & - & $2(16)$ \\
& Mater's degree & $3(100)$ & -- & $5(42)$ \\
& Ed. Spec. & -- & -- & $5(42)$ \\
\hline Total years teaching $M(S D)$ & -- & -- & $10.6(8.8)$ \\
\hline
\end{tabular}

\section{Implementation Fidelity}

This section begins with a description of the adherence and quality ratings, and the results as aggregated across cases and coaches. These data represent the main outcomes associated with implementation fidelity. Brief descriptions of the First Step coaches themselves, including coach level adherence and quality results, can be found in the following sections.

First Step Classroom Check-up Adherence. The coach-completed the Classroom Check-up Process Fidelity Checklist provided as a measure of intervention adherence. Adherence to the four steps of the FSCCU was examined by calculating the percentage of processes completed within and across all tasks. Coach adherence to the 
four steps of the FSCCU process was categorized as Excellent, with $100 \%$ of processes completed across all coaches and cases. Coach reports of adherence on the Classroom Check-up Process Fidelity Checklist was verified by the author, who listened to each audio recorded interview and utilized the checklists as a measure of reliability. Identical results were found.

Coach Motivational Interviewing Quality. The coaches' ability to apply MI, measured using a modified version of MITI, served as the indicator of implementation quality. As can be seen in Table 7, coaches' mean rating across the 5 global domains was 4.16 $(\mathrm{SD}=0.14)$, which is considered proficient. Additionally, mean ratios of reflections to questions were in the Competency range $(M=.82)$, percent open -ended questions ( $M$ $=.46)$, percent complex reflections $(M=40 \%)$ and percent of $\mathrm{MI}$ adherent utterances $(M$ $=97 \%)$ all exceeded the Proficiency threshold. Table 7 also reveals variances between the three coaches. All three coaches met the Competency threshold for global spirit ratings; Coach 1 and Coach 2 reached Proficiency or Competency thresholds for three of the four quality indicators; Coach 3 reached the Competency threshold for one of the four additional quality indicators.

Table 7. Motivational Interviewing Implementation Quality

\begin{tabular}{llllll}
\hline Coach & $\begin{array}{l}\text { Global Spirit } \\
\text { Composite } \\
M(S D)\end{array}$ & $\begin{array}{l}\text { Reflections: } \\
\text { Questions } \\
\text { Ratio }\end{array}$ & $\begin{array}{l}\text { Percent } \\
\text { Open } \\
\text { Questions }\end{array}$ & $\begin{array}{l}\text { Percent } \\
\text { Complex } \\
\text { Reflections }\end{array}$ & $\begin{array}{l}\text { Percent MI } \\
\text { Adherent }\end{array}$ \\
\hline $10(4)$ & $4.00(.56)(\mathrm{C})$ & .81 & $.50(\mathrm{P})$ & $.46(\mathrm{P})$ & $.92(\mathrm{P})$ \\
$11(3)$ & $4.22(.46)(\mathrm{C})$ & $1.18(\mathrm{P})$ & .45 & $.46(\mathrm{P})$ & $1 .(\mathrm{C})$ \\
$60(5)$ & $4.27(.65)(\mathrm{C})$ & .47 & .44 & .29 & $1 .(\mathrm{C})$ \\
\hline$M(12)$ & $4.16(.14)(\mathrm{C})$ & .82 & .46 & $.40(\mathrm{P})$ & $.97(\mathrm{P})$ \\
\hline
\end{tabular}

$n=$ case-load size. MITI Summary Score Competency Thresholds; $\mathrm{C}=$ Competency (highest level); $\mathrm{P}=$ Proficiency. 


\section{Social Validity}

Social Validity Questionnaires. Social validity questionnaires were administered at post intervention to assess both coach and teacher satisfaction with the FSCCU intervention.

Teachers. Of the eight teachers in Wave 2, six (75\%) were highly satisfied with the FSCCU intervention (range 4.00 to 5.00); their satisfaction was classified as strong. The remaining two teachers were somewhat less satisfied (range 3.50 to 3.90 ); their satisfaction was classified as good. Overall, these teacher's responses to the survey represent a high level of satisfaction $(M=4.60, S D=.57)$ and as a group demonstrated strong satisfaction with the FSCCU intervention.

Coaches. Coaches' responses to questions in regards to the FSCCU intervention were as follows: Was the FSCCU compatible with the needs of the teacher? $(M=4.38$, $S D=.52$ ), was the FSCCU intervention effective in teaching effective strategies to deal with challenging behavior? $(M=3.75, S D=.89)$, did the FSCCU intervention have a positive effect on teacher-child interactions? $(M=3.88, S D=.83)$. Teachers' scores suggested they were slightly more satisfied than coaches. Two of the three coaches report satisfaction that can be classified as strong, while one reported satisfaction classified as good.

Focus Group Interviews. Two teachers participated in focus group interviews from each school, except for one school, where three teachers attended. The focus group interviews were scheduled for the week following final data collection procedures at each individual school. The audio recording of these interviews totaled two hours and 38 minutes. Overall, the patterns of these teachers' commentaries were found to converge 
across three broad themes in their perceptions of the FSCCU intervention. First, and that theme which was most prevalent, was labeled Intervention Procedures. Five distinct areas were evident: a) universal principles, b) observation of teacher behavior and resulting data, c) information gathering and feedback sessions, d) burdens associated with the intervention, and e) suggested improvements. With prevalence as the measure, the next most common theme arising from these data was labeled Outcomes. Within this theme teachers commented on two specific areas; the goals that they developed for themselves (or not) as a result of the intervention, and results (teacher outcomes) that they perceived as associated with the FSCCU intervention. Finally, these teachers spoke to a theme encompassing the overall purpose and importance of the FSCCU intervention itself. Within this theme teachers commented on the purpose and importance of positive and negative attention in the classroom, and their own motivation to change their behavior.

Intervention Procedures. The following sections identify the themes present within these data, define the underlying areas touched on by teachers and, organize and present the themes utilizing the teacher's own words.

Universal Principles. The First Step to Success program is grounded on Five Universal Principles of Positive Behavior Support, which are: 1) Define expectations; 2) Teach expectations; 3) Reinforce expectations; 4) Minimize attention for minor inappropriate behaviors; and 5) Have clear consequences for unacceptable behavior (Golly, 2006; Appendix C). Teachers who participated in our research were provided a 1.5 hour introductory training to the universal principles, and a 1.5 hour intervention training which demonstrated the application of these principles within the intervention, and the classroom environment. Even with these two trainings, several teachers expressed 
difficulty remembering these principles. For example, one kindergarten teacher commented: "I remember them on the day, like the day we spoke about them but just not to talk about them in the future..." This teacher's second grade counterpart also reflected difficulty remembering the principles: "Given that I don't even remember what you're talking about right now..," As did two first grade teachers from another school, "I don't remember much about that," and "I don't know, I can't remember."

However, the universal principles were remembered and well received by other teachers, as reflected by this second grade teacher:

I think it's important to focus on what the kids do well. Especially our clientele of kids, I don't think they hear what they do well often - or often enough. So I think it's important for us to remember that just like we like hearing what we do well that they need to hear that as well.

And, by this first grade teacher from a different Elementary School, "This is really part of what we do everyday anyway." Surprisingly, the teachers from a self-contained school for children with severe behavioral and emotional challenges had the universal principles posted in each classroom where the intervention was being implemented. When this group was asked to provide feedback in regard to the principles one teacher simply pointed to the postings and commented, "You could say that we think they are important - Yes." Unfortunately, the focus of the universal principles on positive behavior support did not resonate well with all teachers. A second grade teacher expressed her challenges: The other thing I think and I always struggle with this as a parent and as a teacher, sometimes kids need to do the right thing because they need to do the right thing! They don't need to hear 90 times a day ' $\mathrm{OH}$ I love the way you're sitting in your 
seat." You're just supposed to sit in your seat because you're supposed to sit in your seat! You know? So I think sometimes we give so much positives so, so, so much that they kind of tune out to it, so sometimes it's like, I don't know, I struggle with the balance of that, You know?

This teacher followed up by providing a clearer picture of her struggle in balancing positive and negative attention in the classroom:

...I am not saying that they have to hear the negatives so much, but to kind of back off the positives. So that it becomes, "you do that because you're supposed to do that." Not because you're going to get something, but because you're supposed to do it.

The idea that positive attention should be balanced with negative attention was found in several other comments that teachers made in regards to the universal principles. During one interview with two teachers representing first and second grades, the coach's summary of the teacher's work with the universal principles prompted the following response from the first grade teacher, "...yes, but it's a slippery slope for me." Her counterpart, the second grade teacher commented, "[We're] just trying to find that balance."

Summarizing these teachers' commentary in regards to the universal principles reveals that the two topics addressed by teachers within this area have distinct, and somewhat opposite loci. First for example, given that teachers were provided three hours of training on the universal principles, we find teachers who cannot remember the principles. It may be that the training or methodology utilized to establish the principles more soundly in the teacher's long-term memory was not adequate. Second and likely a 
key summary point within this area is the difference between those teachers for whom the universal principles resonated, and those who were challenged by the reliance on positive behavior support. For some, the universal principles were applicable to their everyday life in the classroom - so much so that the principles were hung on the walls of classrooms in one school. Other teachers spoke eloquently of the need for balance in the use of positive and negative feedback. This need for balance was punctuated with the perception that children should not always receive positive attention for behavior that teacher's expect children should know and be able to demonstrate prior to entering school.

Observation of Teacher Behavior and Resulting Data. A frequent topic of comment by teachers in this area, was the graphs utilized to display the observation of teacher behavior data. Within this topic, several issues were distinguishable including the importance of simplicity, the ease of use, and the meaningfulness of the data.

Numerous comments were made in regard to the importance of simplicity in the display of the data, and the appreciation for the simple nature of the graphs used during the intervention. For example: "It was nice to see it in a nice concrete visual format," and "...Fairly plain and simple," finally "Oh yes, the visual is always better. You can see your positive comments and your negative comments."

Teachers also commented on the relative ease of use allowed for by the graphic presentation of the data. For example the following quotes are from two teachers at the same school: "I honestly kind of just glanced at it. You know I looked at it and just tucked it away in the binder - and that was it!" This teachers counterpart followed by saying: "I did the same, I glanced at the graph and you know from first to second, third 
[observation] and put it in the binder." The following quotes also support the graphic presentation of data and are from two teachers at different schools:

It truly is - whenever you see a visual like that of data that is represented and charted you know that its somebody was taking the time to be precise in what they done, or they would not have charted it - it was not guesstimation.

"And obviously you want your negative comments to be less than your positive comments so I mean just being able to visually see that it is easier."

Most often commented on by teachers within this area, were references to the meaningfulness of data as presented. These comments clustered around the recognition of the teacher's own behavior and the general effectiveness of reviewing this type of behavioral data in graphic form. Several teacher's commented on the recognition of his or her own behavior: "I mean as a pleaser, I enjoyed seeing that it was higher than I expected. You know my positive feedback." A fellow teacher stated, It was, I mean cause sometimes you feel like you nag these kids to death, you know "please sit down, please get this out" and you've asked them a hundred times, so I was please to see that it was a little higher than what I thought it was.

Another teacher added, ...it was also surprising that the actual amount of feedback - when [coach's name] showed me how many per minute - Man! Bang, Bang, Bang! I mean am I doing that? [Coach name] said "yes, and I am not even counting the thumbs up that you're doing" Just my verbalizations!

And another: "I liked looking at them and it seemed to be the positives got more each time, which I liked to see - I have them on a file on my desk. So that is where they are." 
Finally, this conversation between a Kindergarten and First Grade teacher and the interviewer: Kindergarten Teacher, "I think it's important as it holds us accountable, it brought it to my attention - I think, that I probably wasn't doing it enough, even though I thought I was." First grade teacher, "Same here." I mean I was, but not as much as I should have been."

During one focus group, two first grade teachers had the following exchange with the interviewer and each other. These comments support the general effectiveness of the data presented in graphic form:

"Yea, it was like showing improvement. It was like a pat on the back for yourself." "It was a pat on the back, it really was."

"And it did help me change too, I mean seeing that graph and going "whoa." My first one was a pretty big difference there and then by the last one it was the total opposite."

One veteran teacher commented, "It made me more aware, you know after 15 years of teaching I needed a refresher course, so it feels good." This veteran teacher's much younger counter part followed up with the following: "How did it help? [It] showed me that I was improving, but you know, got to keep on, keep it up."

The following quotes came from two second grade teachers from the same school, one having a child who responded well to the intervention, and one having a child who did not. These comments speak to the general effectiveness of the graphs in displaying the data:

Anything visual is best, something that you can glance at - and I've kept them all and I will go over them to pump myself up prior to the beginning of school - I've 
got a couple of rough ones coming I already know I'll need to go over them to know that I can prevent myself from becoming the dragon queen.

"I've saved all mine as well. I liked having the visual."

And at another school,

I wish I could see more of those (graphs) especially towards the end of the year 'cause it's easy to forget. Yes it was effective and it is certainly something I will carry with me at the start of next year.

Information Gathering and Feedback. When teachers were asked to comment on the procedures associated with the FSCCU intervention, two steps of the process were focused on specifically. The information gathering step was commented on by two teachers, who found the questions utilized to prompt discussions (during the interview) too philosophical. Teachers more frequently commented on the feedback step, and nearly all the comments were positive.

The questions asked during information gathering were too "deep" for at least the two teachers at one school who commented,

I think the thing I least liked was that little interview we had to do, you know like your teaching philosophy kind of thing, I felt like I needed to whip out my college paper, I was like “OH” I don't know maybe I wasn't prepared to answer those kind of questions again, it had been so long.

Followed by, "Yes! It was like, let me dig threw all my old college boxes to find that paper." In essence the questions utilized during information gathering may be too complex for the situations teachers find themselves in and made them feel as if back in their senior year in college writing a teaching philosophy paper. 
The feedback step garnered the most response from teachers. These comments uncovered three underlying issues: the realization of their ambivalence, affirmation of their ability, and differences from other forms professional development. One revelation teacher's experienced during the feedback interview was the realization that they could do better. While other teachers commented that the feedback interview fortified what they already knew about their abilities and was provided in a strengths based fashion, which may have been different than their typical experience. For example, teachers who felt as though the feedback step provided information that was a surprise, often commented on the disappointment they felt upon realizing the difference between their perceptions (about their positivity) and what the data revealed:

I know that when [coach's name] came in to observe I really looked at the paper that she gave me cause I was disappointed, I'd always thought of myself as a positive person but depending on the kind of personality of your class and how we perceive the personalities of the class I found that I was not quite as positive as I thought I was and that was an eye opener for me, I became even more conscious of it, even after she left- even now- as I was not as positive as I thought I was with the others.

Another teacher commented, It was an eye opener for me, I thought I was more positive than I was - not that I thought it was real bad - still I thought I was more positive. I tend to be one that picks on little things - instead of picking my battles I don't always pick them as well as I should. So that was revealed in there and that made me stop and think do I want to get on them about that or not. So it was good for me. 
This teacher compared the data shared by the coach with other information she had read on the subject:

It's not anything that was like "oh no, I hate this part" but I guess seeing initially the first one, it was pretty neck and neck and I had heard or read or been told how many positives you are supposed to have rather than negative, and I was not doing that - and probably still not doing that.

Finally, the following,

Yes, I looked at it like a coach. A coaching situation, and um...because I had one view of myself it was interesting and I hate to say, because she never was negative, you didn't do this you didn't do that, it was like an AH HA moment, for me. I mean I have had a lot of those this year.

Other teachers felt the feedback step portrayed them for what they knew they were, and expressed gratitude for the reinforcement the feedback step provided: "It was motivational, having someone else reinforce what it is that you are hoping you are already doing." And another teacher: "You felt so good about what you were doing, that you were doing something right and that someone was seeing what you were doing." Still other teachers commented on the way in which the feedback session was presented; a non-threatening, strengths based approach resonated with teachers:

I didn't feel as if he was judging me, I felt as if he was concerned about the child, and was like "hey I am here to help you with this child" I didn't feel like was saying “[Teacher's name] you're horrible, or you're great or whatever. I never felt that way at all.

Another teacher commented, 
I don't know that he approached it as discouraging, he just said, "here's what you doing" and I didn't like it [the results]. I didn't like the fact that I was getting more negative than positive. I didn't realize that I was doing it - it was an eye opener.

Followed by, "[The coach] showed me - like "here is what I observed" and then said "what do you think of it?"

One set of comments between two teachers at the same school, helped bring to light that the type of feedback used with the FSCCU may have been different than the feedback these teachers had received from other forms of professional development:

"It was motivational, having someone else reinforce what it is that you are hoping you are already doing."

"It was done in a non threatening kind of way..."

"Unlike some people in this building."

"Because at the core most of us want to do well, and what is right. Sometimes that gets lost in the everyday shuffle and what not..."

Burden. Teachers who participated in focus group discussions were asked to reflect on the length and time demands associated with the meeting and data review required for the FSCCU intervention, in the context of their classrooms. This particular theme was commented upon the least by teachers; no procedures or time requirements were commented on as burdensome by any of the participating teaches. For example, ...it was minimal, we were doing what we were doing it was not like we had to change what we were doing. I mean 5 minutes 10 minutes to talk about it afterwards... and she usually came right after planning so that was perfect. 
Time requirements for the maintenance observation step were also noted as minimal as revealed in this discussion between two first grade teachers at one school:

"I mean she was very accommodating in terms of giving the graphs, I mean she would just kind of slip them, leave them, and we didn't, I didn't mind that, there wasn't a whole lot of conversation as far as that goes the way she did it."

"She would kind of make a note at the bottom, which was helpful. It wasn't something that had to be sat down and discussed."

"She just said "Any questions?" She was open for it, but it wasn't like we had to sit down and have a long conversation, which I appreciated. I like the way that was handled. Do with it what you want. I appreciated that."

One teacher commented on the paperwork requirements for the intervention: "The paperwork was minimal."

Overall the burdens associated with the FSCCU intervention were minimal. Teachers who participated in these focus groups reflected that sentiment in their comments when asked directly.

Suggested Improvements. Teachers were asked a variety of questions to elicit their suggestions for improving the current intervention procedures. Three of the questions were quite specific and asked for the teachers' opinions on three proposed changes to the system utilized during their participation to code observations in preparation for feedback to the teacher. In addition to solicited comments regarding possible coding scheme changes, multiple unsolicited comments were captured and are provided to end this section, 
When asked about the possibility of receiving feedback in regards to their use of behavioral specific vs. general feedback with children, the majority of teachers agreed this information would be helpful, and at least three of these teachers provided reasons for their opinion: "Cause I know that I struggle as a teacher as I give a lot of general feedback...I try to give specific. That would be good.” Another teacher commented, I try to give specific feedback when one [child] is doing what you want and one is not "I really like the way [child's name] is...and you don't give a lot of that unless there are a lot of people who are not doing what they should.

Finally, another teacher commented, I give specific feedback but I'm just not doing it unless I really want all the kids to behave. But as far as if everyone is doing a good job then I'm like "oh you guys are doing a great job.

Although the majority of teachers approved of a change in the observation coding scheme to provide information on behaviorally specific vs. general feedback, two teachers felt this information would only be as useful as they found the current information to be:

I think in an ideal world I would love to see the difference between those things, in reality I probably would not give it much more of a look that what I gave that [points to original graphs]. I mean, [I'm] just being honest.

Followed by, "I would agree with that, Ideally that would be great if that was our number one focus when we are here, you know, but unfortunately it's not..." When posed with the possibility of receiving information on their use of non-verbal feedback as well as verbal feedback, teachers responded positively. The following are selected examples, each from 
a different school, representing only a few of the numerous positive responses to the suggested change: "Definitely. Honestly that's probably half your positives. For me it is. I am constantly hugs, smiling, and thumbs up." From another school, "Yea, I am like a third base coach, giving signs." Then another, "Oh, I think that would be good. I think that would be a positive thing to add." Finally, the following, "I do a lot of non-verbal and it would be great to be recognized for that." One teacher highlighted the difficulty of capturing non-verbal feedback:

Knowing that you had the right personnel to evaluate what is and what is not a positive (non-verbal feedback) I'd say it would be ok. I think that you need to make sure that the observers are well trained at it because that [distinguishing non-verbal feedback] would be the biggest challenge.

When asked about a coding scheme that only included the focus child, excluding peers and the class as a whole from the observation of teacher behavior coding scheme; teacher's felt this change would limit the effectiveness of the data and may be detrimental to the intervention as a whole. For example, most teachers commented that the child is a part of the greater classroom, and should not be singled out. The following was a typical response and sentiment to this prompt, "How could you do that? As the child is part of the bigger picture too." As well, teachers felt as thought the contingency for peers to provide positive feedback to the focus child might be compromised. "I also feel that it might take away part of what was effective in the program and that was allowing other kids to be positive with him [the focus child]."

Many teachers offered unsolicited suggestions. At one elementary school a first grade teacher and her second grade counterpart suggested more frequent observations and 
the possibility of follow-up observations. The first in regards to the quantity of observations, "I would not mind if you even did more." Her counterpart agreed, "That's what I was thinking - I wouldn't mind more at all. It's helpful!" Form another school, this comment: "I would not even mind if when the program was over if there was like a follow-up - Like sometime within that school year, so that you can check to see if I am still keeping up with my goals."

These two teachers, from different schools, offered similar suggestions relating to the scope and sequence of behavioral targets for the observations:

I think it may be better if you did like, you know the first time you could give positive and negative [results] for that [focus] child and the class, and then the next time do specific and general feedback. I don't think you should do all three of those at once. It would be too much.

This comment from a different school: "I like the idea of adding elements as you go along. So first observe for one thing, then add another, then another."

Outcomes. Teachers were asked to comment on any personal goals set for themselves as a result of the intervention, and if the intervention had an affect on their attitude towards or relationship with the focus child, other children, or the classroom climate in general. Teachers were also prompted to reflect on how the intervention was or was not effective in changing the focus child's behavior in the classroom.

Personal Goals. Some teachers who responded to this question prompt could not recall the specific goal they had set as a result of the initial feedback interview. For example, the following two teachers answered vaguely, "I think I said something to her, but I can't remember." Followed by, "I can't remember if I said that to her or not. And I 
don't know that I specifically wrote it down as goal..." Others named their goals specifically, and may have provided rationales for developing their goals: "I had to focus on minimizing attention for minor inappropriate behavior. I had to keep that one in my mind the most as I don't always do that one." Another teacher said: "Generally my goals were to increase positives and I think I obtained them; I don't think I set a particular number, I was just looking for improvement." Finally, My goal was to try not to verbally give negative feedback. To kinda just do the tapping of the shoulder. Just walk around the room and praise each child, and if they were not doing what they were supposed to be - to just kinda touch them on the shoulder and then when they were - to praise them.

Teacher Outcomes. Teachers responded to this question prompt with examples of improved relationships with the focus child: "It changed my relationship with my child, cause I noticed the more I gave him positives he would smile - and it was like I wanted to cry." Another teacher said: "I think with my student we understood each other a bit more, we paid more attention to each other. It did help our relationship." One teacher spoke of acceptance,

It did make me focus more on the child and accept him and his behaviors, it did make me think about what I was saying and doing and giving the opportunity to make sure that I said the positives and that I looked at him differently cause he was a very challenging - they are hard to get to know and have a relationship with. While another spoke of awareness, "For me it gave me an awareness of my whole class, to try to bring it as much as possible... and there I am giving more complements and 
thumbs up and like that." Additionally, teachers remarked on the improvements in the use of behaviorally specific positive feedback:

...I explained explicitly what this child was doing - I mean I didn't say what this group was doing something, I said "look at the way [child] is sitting, she is sitting there with her hands in her lap. [Child's name] can you teach everyone who is here how to sit at this table?" I mean I explicitly said and worked on one thing, and that was a remarkable event.

While another teacher said,

It also reminds you to be specific in your feedback and we should remind each other of that more - weekly or daily of the importance of that I mean good job class is a positive but good job class lining up today in a nice quiet line I really do appreciate that - then they know exactly what you are talking about and being vague does not mean that it is a good - it is a positive so you've got to be precise and I forget about those things.

Several teachers had difficulty recalling their personal goals, while others related their goals and the rationales behind creating them. Teacher outcomes that were provided were positive, often focused on the improved status of their relationship with the focus child.

Purpose and Importance. Focus group interview prompts included questions designed to elicit the teacher's perception of the overall purpose and importance of positive and negative feedback with children, and to determine the extent to which teachers believed this was important or not, and why. Many teachers answered in the 
affirmative; that positive attention was foundational, and worked to bolster children's abilities:

Well, in first grade you want to build them up, you know, and give them a lot of confidence. So they are not intimidated, by learning to read or learning to something new in math. Really build them up so they are ready to tackle new things. Just 'cause something is new and scary looking, we don't want them to be afraid - tell them “you're good at this, come on let's think about it.

Other teachers answered this question prompt with caution. Particularly teachers from a self-contained school for children with severe emotional and behavioral disorders; they warned of the overuse of positive feedback with children who's goal is to return to less restrictive environments:

What's important is to fade any positive attention as the year progresses. We try to fade positive attention so that at the end of the year you are not giving them constant feedback...Not to fade it all away but fade it to the level of a regular classroom...

Another teacher agreed,

I agree with what she said. We do try to fade away and try to challenge them to the point that they will be challenged when they get to a comprehensive school. Even in a self-contained environment, because there the attention won't be there as much...

Some teachers reflected their belief that children should know how to behave and do not necessarily need to be reinforced for behaviors that are expected: 
I think you definitely need positive, I try to stay three to one, I am not sure if that pans out, some kids you can say all day long "Oh I Like how so-in-so is doing this" and some kids will never do what so-in-so is doing but for the most part I do like to keep it positive in fact if I can to try to week out the negatives.

Another teacher questioned this practice as well: "...I mean you can say "I like how [child's name] is standing in line" only so many times before you have to go right to them and say "behave yourself!" Following this, one teacher gave an example of the focus student from her classroom:

My specific student in the beginning seemed to do well with the positives, but then she was able to manipulate it to some extent and then it was like the positive stopped working. As I was trying to work with her and figure out what worked that was frustrating and I know that I got frustrated and I was like "I'm not going to go out of my way to give her positive, positive, positive I mean I still did it for the class but I did not you know, because she just figured out the way to manipulate it, so that is just my specific circumstance.

Teachers who responded to this question prompt addressed the importance of the feedback for children in their classrooms positively, yet with caution. Issues reflected by the teachers included the importance of balance in the use of feedback, particularly caution in the overuse of positive feedback. As well, a number of teachers seemed to question the need to provide positive feedback to children for behaviors that are expected.

Teachers provided open and honest responses during these interviews that were positive on the whole, and found to be most prevalent within the themes of intervention 
procedures, outcomes, and the overall purpose and importance of their use positive and negative feedback.

\section{Functionality}

This section presents case-level and aggregate results to better understand whether the intervention functioned as intended and consistent with the logic model presented in Chapter 3. The case-level summaries include individual child and teacher characteristics, and are constructed from: (a) standardized questionnaires completed by coaches, teachers and parents; (b) coach-completed case summary files; (c) CLASS monitoring logs; and (d) observations of child and teacher behavior. Using these data sources, the FSCCU Analysis Classification System (See Table 4, Chapter 3) was employed to categorize cases related to the following variables: (a) MI implementation quality, (b) coach-teacher alliance, (c) teacher-child relationship, (d) teacher perceptions of children's social skills and problem behaviors (See Table 10), (e) observations of children's academic engagement (See Table 10), and (f) observations of teacher's use of positive and negative feedback. Case-level outcome results are tabularized in Appendix M for convenience. An aggregate level analysis is then presented to assess patterns across cases, both with regard to individual variables and interactions among variables and related to the elements of the logic model (See Chapter 3, p. 60).

Case-level Analysis. In this section case summaries are utilized to provide case level results for coach- teacher- and child-related elements of the logic model. Each case summary begins with a brief description of the focus child followed by the results of a review of the First Step CLASS Monitoring Log (See Appendix I), which includes the calculation of the intervention dosage (calculated as the proportion of program days 
delivered by the coach and teacher out of 30 possible) and compliance (calculated as the proportion of days when the focus child met the daily point criteria). Next, results from the Coach and Teacher Alliance Survey (See Appendix G), Observation of Teacher Behavior (See Appendix N), Enhanced First Step CLASS Component Fidelity Checklist (See Appendix H), and Student Teacher Relationship Scale (See Appendix J) are presented. To provide case-level information, and to determine if the change in teacherchild relationship is statistically reliable, the Reliability Change Index (Jacobson \& Truax, $1991 ; \mathrm{RCI})$ was calculated. The RCI takes into account the test-retest reliability of the STRS, provides an indication of cases that have responded to the intervention, and "...provide[s] a precise method for classifying clients as "changed" or "unchanged" on the basis of clinical significance criteria" (Jacobson \& Truax, 1991, p. 13)." The RCI is calculated on an individual case basis. A significant RCI statistic $(>/=1.96)$ indicates a reliable change in the teacher's perception of their relationship with the focus child. This is followed by the presentation of child outcomes for AET, Social Skills, and Problem Behaviors. Each case summary ends with a brief appraisal of the logic model's functionality given the specific results of the case.

Child 1100. This child is a six-year-old Caucasian boy, whose teacher is referred to as 5030 . According to parent report, this child was in the process of being diagnosed with bipolar, oppositional defiant, and obsessive-compulsive disorder while participating in the study. In addition to the support of the First Step coach, this child received multiple services from community-based behavioral and mental health providers during the study, including; a local psychiatrist, state mental health services, and a comprehensive evaluation from a local child evaluation center. A review of the First Step CLASS 
monitoring log indicates that this child completed the 30 -day intervention with only 1 recycle day- a day on which the child did not meet the behavioral expectations of the intervention. No significant irregularities in the intervention sequence were found, and no program modifications were necessary. This resulted in an intervention dosage of .97 , with 97 percent compliance on the part of the child.

Both teacher and coach rated the coach-teacher alliance as strong; the coach's alliance rating was $4.0(S D=.00)$ while the teacher's was $5.0(S D=.00)$. During the intervention, teacher 5030 committed to increasing the rate of praise used in the classroom. At baseline, Teacher 5030 demonstrated a unit rate of positive to negative feedback of 3.87 , but did not maintain the high ratio through the maintenance phase of the FSCCU intervention, and was classified as a non-responder at post intervention (unit rate $=2.12$ ). However, her implementation of the First Step CLASS component as rated by independent observers was classified as excellent for intervention adherence (.97) and quality (.93). Calculation of the Reliability Change Index, indicates a reliably significant reduction in the teacher's perception of conflict in her relationship with the child was found at post intervention $(\mathrm{RCI}=5.85)$, while increased closeness was also reported, it did not reach a reliably significant level $(\mathrm{RCI}=1.32)$.

The coach reported that this child responded to the intervention amazingly well, considering his mental health issues and history of very challenging behavior. Outcome results for this child support the coach's report with reliably significant improvement in social skills $(\mathrm{RCI}=9.87)$, and decreased problem behaviors $(\mathrm{RCI}=-8.64)$. Furthermore, this child maintained his $\mathrm{AET}$, at baseline $(\mathrm{M}=.86, \mathrm{SD}=.05)$ and post intervention (M $=.89, \mathrm{SD}=.12$ ) well within the normal range. 
From the perspective of the logic model, the coach's competent use of MI, strong coach and teacher alliance, positive change in teacher perception of conflict, and teacher's strong adherence and quality scores for the implementation of the CLASS component supported the pathways of influence proposed within the logic model. However, the FSCCU intervention was not sufficient to enable the teacher to maintain desired unit rates of positive to negative feedback, as the teacher's baseline ratio was relatively high.

Child 1106. This child is a seven-year-old African American boy, whose teacher is referred to as 5028. During the intervention, he received special education services for an emotional behavioral disorder, and support from a school-based mental health therapist and psychiatrist, who monitored his medications. A state mental health service worker coordinated these services. Both the therapist and service coordinator worked with child's Mother in her home. A review of the First Step CLASS monitoring log indicated this child completed the 30-day intervention cycle with five recycle days. On day eight of the intervention he was placed in a Crisis Stabilization Unit (CSU) for eight nights following an attempt to harm himself. The CSU recommended hospitalization unless the family would agree to intensive in-home therapeutic services. Staff from a family restoration program was assigned to work in the home two to three days a week. The First Step intervention was modified after this event to include individualized reward contingencies. This crisis situation resulted in an intervention dosage of .56, with $77 \%$ compliance on the part of the child, during the intervention.

Both teacher and coach rated the coach-teacher alliance as strong. The coach's alliance rating was $4.0(S D=.53)$ and the teacher's $5.0(S D=.00)$. During the 
intervention, the teacher committed to improving the rate of praise utilizied in the classroom as a goal for the program. At baseline, this teacher demonstrated a unit rate of positive to negative feedback of 1.66 , improved through the maintenance phase, and was classified as a responder to the FSCCU intervention (unit rate $=3.16$ ). Her implementation of the First Step CLASS component was classified as excellent for intervention adherence (.95) and adequate for implementation quality (.89). Calculation of the Reliability Change Index established that no reliably significant reduction in the teacher's perception of conflict in her relationship with the focus child was found at post intervention $(\mathrm{RCI}=-.84)$, while a small increase in closeness was also reported it did not reach a reliably significant level $(\mathrm{RCI}=.19)$.

The First Step coach reported the effectiveness of the intervention was inconsistent for this child. However, outcomes for this child indicate the CLASS component was successful, as evidenced by reliably significant improvements in social skills $(\mathrm{RCI}=10.12)$, and decreases in problem behaviors $(\mathrm{RCI}=-2.88)$. Furthermore, this child maintained academic engagement at baseline $(M=.81, S D=.08)$ and post intervention $(M=.80, S D=.15)$ well within the normal range.

From the perspective of the logic model, the coach's competent use of MI and strong coach and teacher alliance supported the pathways of influence proposed within the logic model. Unfortunately, the lack of significant change in teacher perception of conflict or closeness does not. However, the teacher's responsiveness to the FSCCU intervention and more than adequate CLASS component implementation fidelity may have contributed to the child's responsiveness to the intervention overall, even in light of low intervention dosages and the child's crisis during the intervention. 
Child 1109. This child is an eight-year-old Caucasian boy, whose teacher is referred to as 5029. This child has been diagnosed with Asperger's Syndrome. According to teacher and parent reports prior to the intervention, he tantrumed frequently and experienced obsessions and compulsions which impaired his social and behavioral interactions. In fact, both this child's parent and teacher reported these obsessions often leave him completely unable to continue normal daily activities. During the intervention, he was receiving behavioral therapy and psychiatric services from a Community Mental Health organization. A review of the First Step CLASS monitoring log indicated he completed the 30-day intervention with 4 recycle days, which resulted in an intervention dosage of .87 and $87 \%$ compliance on the part of the child. No significant irregularities in the intervention sequence were found, and no program modifications were necessary.

Both teacher and coach rated the coach-teacher alliance as strong. The coach's and teacher's average alliance ratings were 4.25 and $5.0(S D=.00)$, respectively. During the intervention, this child's teacher committed to increasing positive feedback as a goal for the program. At baseline, the teacher demonstrated a unit rate of positive to negative feedback of 2.66; this rate improved over the course of the maintenance phase and the teacher was classified as a responder to the FSCCU intervention at post intervention (5.66). As well, her implementation of the First Step CLASS component as rated by independent observers was classified as excellent for intervention adherence (1.0) and adequate for implementation quality (.88). Calculation of the Reliability Change Index indicates a reliably significant reduction in the teacher's perception of conflict in her relationship with the focus child was found at post intervention $(\mathrm{RCI}=-2.79)$. While 
increased closeness was also reported, it did not reach a reliably significant level $(\mathrm{RCI}=$ $1.70)$.

The child's First Step coach reported the intervention was very effective for this child, particularly the child's responses to the Green card. Outcome results for this child supported the coach's perception of effectiveness with reliably significant improvements in social skills $(\mathrm{RCI}=7.34)$ and decreases problem behaviors $(\mathrm{RCI}=-3.52)$. Furthermore, this child maintained an average academic engagement at baseline $(M=.84, S D=.11)$ and post intervention $(M=.86, S D=.08)$ well within the normal range.

From the perspective of the logic model, considering the coach's competent use of MI, strong coach and teacher alliance, positive changes in teacher's perception of conflict and closeness, and the teacher's responsiveness to the FSCCU intervention all support the pathways of influence proposed within the logic model. Furthermore, this teacher's CLASS component implementation fidelity was more than adequate and may have contributed to the child's responsiveness to the intervention overall.

Child 1117. This is a seven-year-old Caucasian boy, whose teacher is referred to as 5022. According to teacher report, he was diagnosed with Attention Deficit Disorder, and displayed significant anger management issues prior to the intervention. His tantrums often included falling to the ground, kicking, head banging, poking himself with pencils and other sharp objects, and demanding food, according to teacher report. A significant number of behavioral referrals had resulted in multiple suspensions since kindergarten. This child had received services in the previous school year from a local mental health organization, but was not receiving these services during the intervention. A review of the First Step CLASS monitoring log indicated this child completed the 30-day 
intervention cycle in 31 days with one recycle day. This resulted in an intervention dosage of .97, with $97 \%$ compliance and no modifications. The First Step coach reported very impressive results over the course of the intervention.

During the intervention, his teacher committed to decreasing the amount of negative feedback she used in the classroom. At baseline, this teacher demonstrated the lowest unit rate of positive to negative feedback of all teachers in the study (0.2). Although she did improve, she was not able to meet the desired ratio at post intervention, and was classified as a non-responder (unit rate $=1.8$ ). This teacher's implementation of the First Step CLASS component was excellent for intervention adherence (.96) and adequate for implementation quality (.82). Calculation of the Reliability Change Index indicates a reliably significant reduction in the teacher's perception of Conflict in her relationship with the child at post intervention $(\mathrm{RCI}=2.23)$, although improvements in her perception of Closeness were not large $(\mathrm{RCI}=.19)$.

Outcomes for this child indicate reliably significant improvements for social skills $(\mathrm{RCI}=7.59)$, and decreases in problem behaviors $(\mathrm{RCI}=-12.48)$. Furthermore, this child's average academic engagement was well below normal ranges at baseline $(M=.60$, $S D=.16)$, and improved to well within the normal range at post intervention $(M=.81$, $S D=.06)$.

From the perspective of the logic model, the coach's competent use of MI and strong coach and teacher alliance support the pathways of influence proposed within the logic model, while the lack of significant change in teacher perception of conflict or closeness and the teacher's non-responsiveness to the FSCCU intervention did not. This 
teacher demonstrated adequate CLASS component implementation fidelity, which may have influenced this child's responsiveness to the intervention overall.

Child 1123. This child is a five-year-old Caucasian female whose teacher is referred to as 5031. Her parents report that behavioral concerns were significant at home and in school prior to the intervention. Her teacher reported that when angered, she would become very upset and resort to crying for long periods of time prior to the intervention. A review of the First Step CLASS monitoring log indicates this child completed the 30day intervention cycle with two recycle days resulting in an intervention dosage of .93, with 93 percent compliance. The only intervention modification necessary was shortened daily intervention intervals.

During the intervention, this teacher committed to increasing her positive feedback and decreasing negative feedback to children in her class. This teacher responded to the FSCCU intervention; her baseline unit rate of positive to negative feedback was 1.29 , which improved to 5.88 during the maintenance phase. The teacher implemented the First Step CLASS component with ratings by independent observers classified as adequate for intervention adherence (.87) and quality (.83). A reduction in the teacher's perception of conflict in her relationship with the focus child was found at post intervention, as was increased closeness, although calculation of the Reliability Change Index indicated that neither reached a reliable level of clinical significant level $(\mathrm{RCI}=1.40$ and 1.70 respectively $)$.

The First Step coach reported the child was responsive to the intervention. Outcomes for this child were not reliably significant for social skills $(\mathrm{RCI}=-.51)$ or problems behaviors $(\mathrm{RCI}=1.6)$. However, this child's average academic engagement at 
baseline was below normal ranges $(\mathrm{M}=.54, \mathrm{SD}=.14)$, and improved to within normal ranges at post intervention $(\mathrm{M}=.82, \mathrm{SD}=.14)$.

From the perspective of the logic model, the coach's competent use of MI and strong coach and teacher alliance supported the pathways of influence proposed within the logic model; the lack of significant change in teacher perception of conflict or closeness did not. The teacher's responsiveness to the FSCCU intervention and adequate CLASS component implementation fidelity was not sufficient to influence child outcomes in the areas of social skills or problem behaviors, although improved academic engagement was found at post intervention.

Child 1128. This child is a five-year-old Caucasian male whose teacher is referred to as 5033. Prior to intervention this child was reported by parents to be destructive in the home (e.g., breaking items and damaging walls). At school, his teacher reported the child did not following directions, had difficulty interacting with other children, refused to complete his work, struggled with keeping his hands to himself, and often became disruptive. A review of the First Step CLASS monitoring log indicated he completed 23 of the 30-day intervention cycle, with seven recycle days, as he and his family moved prior to the completion of the intervention. This resulted in an intervention dosage of .77, with 77 percent compliance on the part of the child. Modifications included individualized behavioral expectations and shortened daily intervention intervals.

During the intervention, this teacher committed to increasing positive feedback and decreasing negative feedback used with the children in her class. At baseline, the teacher demonstrated a low unit rate of positive to negative feedback (0.38). Although improved, she was not able to meet the desired ratio during the maintenance phase, and 
was classified as a non-responder with a unit rate of 2.84 . This teacher's implementation of the First Step CLASS component was classified as excellent for intervention adherence (.90) and adequate for implementation quality (.76). As well, this teacher reported a reliably significant increase in her perception of closeness with the focus child at post intervention $(\mathrm{RCI}=3.21)$, and decreased conflict, although not at a reliably significant level $(\mathrm{RCI}=0.840)$.

As reported by the First Step coach, the child responded moderately well to the intervention, with reliably significant improvements in social skills $(\mathrm{RCI}=7.59)$ and decreases problem behaviors $(\mathrm{RCI}=-5.76)$. As well, this child improved his academic engaged time from below normal ranges $(\mathrm{M}=.61, \mathrm{SD}=.25)$ at baseline to well within a normal range at post intervention $(\mathrm{M}=.88, \mathrm{SD}=.04)$.

From the perspective of the logic model, the coach's competent use of MI, strong coach and teacher alliance, and the positive change in teacher perception of closeness, supported the pathways of influence proposed within the logic model; the FSCCU intervention was not sufficient to allow the teacher to significantly increase rates of positive to negative feedback. This teacher's CLASS component implementation fidelity was adequate and may have contributed to the child's responsiveness to the intervention overall.

Child 1144. This child is an eight-year-old Caucasian boy, whose teacher is referred to as 5021. According to parent report, he is diagnosed with Attention Deficit Hyperactive Disorder and is medicated for symptoms related to this diagnosis. His teacher reported he is very inattentive to classroom and social structures, often fixating on small toys or materials (e.g., erasers, paper clips) to the extent that he is unable to 
complete typical classroom tasks and activities. In the previous school year, he received services from a local mental health organization. A review of the First Step CLASS monitoring log indicates this child completed only 15 of the 30-day intervention cycle, with eight recycle days. This resulted in a low intervention dosage of .50 , with only 53 percent compliance on the part of the child during days the intervention was implemented. During the intervention according to a coach completed case summary, the child was experiencing divorce, poverty, and a harsh often oppressive parenting style. Multiple modifications were attempted including the identification of other adults in the school who provided additional reinforcement, modified daily intervention intervals, and various reinforcement strategies.

During the intervention, Teacher 5021 committed to increasing the amount of behavior specific positive feedback she used with children in the classroom. At baseline, this teacher demonstrated a unit rate of positive to negative feedback of 1.18 , which improved during the maintenance phase, and was classified as a responder to the FSCCU intervention (unit rate $=3.44$ ). Furthermore, her implementation of the First Step CLASS component was classified as excellent for intervention adherence (.96) and adequate for implementation quality (.89). Calculation of the Reliability Change Index indicates no reliably significant change in their teacher's perception of her relationship with this child. Although not reliably significant, the teacher's perception of conflict in her relationship with the child was improved at post intervention $(\mathrm{RCI}=1.12)$, yet this teacher's perception of closeness with the child fell during the intervention $(\mathrm{RCI}=-1.89)$.

The First Step coach reported that the child's responsiveness to the intervention was significantly impaired by the child's life circumstances. Outcomes for this child 
indicated that reliably significant improvements were found for social skills $(\mathrm{RCI}=4.05)$, but not problem behaviors $(\mathrm{RCI}=.32)$. Furthermore, this child's average academic engagement at baseline $(M=.66, S D=.17)$ was well below normal ranges, and did not improve at post intervention $(M=.68, S D=.26)$.

From the perspective of the logic model, the coach's competent use of MI and strong coach and teacher alliance support the pathways of influence proposed within the logic model, while the lack of significant change in teacher perception of conflict or closeness did not. Although the teacher was classified as a responder to the FSCCU intervention, the child's problem behaviors did not decrease, and academic engagement did not improve. Thus, despite adequate implementation, the intervention was not sufficiently powerful in this situation.

Child 1163. This child is an eight-year-old African American female, whose teacher is referred to as 5038. According to parent report prior to the intervention, she was stubborn, liked to control others through yelling, hitting and throwing objects in defiance. At school the teacher reported that the child "has an attitude," did not follow directions, had difficulty cooperating with other girls in the class, "tested" the teachers patience. A review of the First Step Monitoring Log indicated the child completed the 30day intervention cycle with only three recycle days, and one day on which the teacher did not implement the intervention or that schedule conflicts did not allow. This resulted an intervention dosage of .86 , with $90 \%$ compliance. Only minor program modifications were necessary including individualized reward structures, and a surrogate for individual attention. 
Both teacher and coach rated the coach-teacher alliance as strong. Coach and teacher's alliance ratings were $5.00(\mathrm{SD}=.00)$ and $4.88(\mathrm{SD}=.35)$, respectively. During the intervention, this teacher committed to increasing specific positive feedback to the children in class as a goal for the program. The teacher improved her rate of positive to negative feedback from 4.44 at baseline to 20.00 during the maintenance phase, and was classified as a responder. This teacher's implementation of the First Step CLASS component was classified as excellent for intervention adherence (1.00) and adequate for implementation quality (.89). This teacher's perception of conflict in her relationship with the focus child increased at post intervention $(\mathrm{RCI}=1.40)$, while her perception of closeness decreased $(\mathrm{RCI}=-1.70)$. Calculation if the Reliability Change Index indicates that neither score was reliably insignificant.

The First Step coach reports the child responded reasonably well to the intervention during the first 15 days, but her interest faded during the final 15 days. Outcome results for this child support the coach's perception of effectiveness with no reliably significant improvements found in social skills $(\mathrm{RCI}=.25)$ or problem behaviors $(\mathrm{RCI}=.32)$. This child maintained an average academic engagement at baseline $(\mathrm{M}=.87$, $\mathrm{SD}=.09$ ) and post intervention well within the normal range $\mathrm{M}=.80, \mathrm{SD}=.01$ ).

From the perspective of the logic model, the coach's competent use of MI and strong coach and teacher alliance supported the pathways of influence proposed within the logic model, while increases in teacher perception of conflict and decreases in teacher perception of closeness did not. The teacher's responsiveness to the FSCCU intervention was significant. More than adequate CLASS component implementation fidelity was not 
sufficiently powerful to support responsiveness to the overall program, as two of three child outcomes lacked significant change.

Child 1164. This child is an eight-year-old Caucasian male, whose teacher is referred to as 5039. This child had been diagnosed with ADHD, but was not medicated and received no community support during the intervention. At home, his parents reported the child had difficulty staying focused long enough to complete his home and school work; at school his teacher reported he was continually "out of his seat," became frustrated easily, distracted other children, and frequently talked out loud at inappropriate times. A review of the First Step Monitoring Log indicated the child completed the 30day intervention cycle with only one recycle day, and six days that the teacher did not implement the intervention or that schedule conflicts did not allow. This resulted in an intervention dosage of .77, with $95 \%$ compliance. No significant modifications were attempted.

Both teacher and coach rated the coach-teacher alliance as strong. With the coach's rating of $4.75(S D=.46)$ being slightly less than that of the teacher's $(M=5.0$, $\mathrm{SD}=.00)$. During the intervention, this teacher committed to increasing specific positive feedback to the children in class as a goal for the program. At baseline, the teacher demonstrated a high unit rate of positive to negative feedback (3.72), and improved through the maintenance phase. This teacher was classified as a Responder to the intervention with a unit rate of 5.00 at post intervention. As well, this teacher implemented the First Step CLASS component with ratings by independent observers classified as excellent for intervention adherence (.93). Unfortunately, her implementation quality suffered with a classification of poor (.68). Calculation of the 
Reliability Change Index indicates no reliably significant change, in that this teacher perceived slightly more conflict $(\mathrm{RCI}=1.12)$ and slightly less closeness $(\mathrm{RCI}=-0.57)$ in her relationship with the focus child at post intervention.

Both teacher and First Step coach reported the child had responded very well to the intervention. Outcomes for this child indicate the intervention was successful as reliably significant improvements in social skills $(\mathrm{RCI}=2.28)$ were found. No change was recorded from baseline to post intervention for problem behaviors $(\mathrm{RCI}=.00)$. This child maintained an average academic engagement at baseline $(\mathrm{M}=.84, \mathrm{SD}=.01)$ and post intervention $(\mathrm{RCI}=.82, \mathrm{SD}=.07)$ well within the normal range.

From the perspective of the logic model, the coach's competent use of MI and strong coach and teacher alliance supported the pathways of influence proposed within the logic model, while the lack of significant change in teacher perception of conflict or closeness did not. The teacher's responsiveness to the FSCCU intervention and excellent adherence to the CLASS component implementation was counterbalanced by poor quality of implementation. Nonetheless, child outcomes were positive for two of three child outcomes, and the child was classified as a partial responder.

Child 1170. This child is a seven-year-old African American boy, whose teacher is referred to as 5042. Prior to the intervention, this child's stepmother reported that he could not be left alone or unsupervised for even short periods of time, as he destroyed his and others' belongings. Additionally, his teacher reported he became aggressive when dealing with any behaviors he that perceived as intruding on his space or belongings. The First Step CLASS monitoring log was not returned by the teacher (who has left employment with the school) and was unavailable for review. However, the case report 
for this child indicates frequent recycle days, and modifications to the intervention including individualized reward contingencies, and the identification of other adults in the school who provided additional reinforcement. Both teacher and coach rated the coach-teacher alliance as strong. With the coach's alliance rating was $4.75(\mathrm{SD}=.46)$ while teacher rating was $5.0(\mathrm{SD}=.00)$. During the intervention, his teacher committed to improving the amount of behavior specific positive feedback she utilized with children in the classroom. At baseline, this teacher demonstrated a unit rate of positive to negative feedback of .88 , which improved during the maintenance phase, although not to the target objective, which classified her as a non-responder (unit rate $=2.18$ ). This teacher implemented the First Step CLASS component with ratings by independent observers classified as excellent for intervention adherence (.97) and adequate for implementation quality (.75). Calculation of the Reliability Change Index indicates a reliably significant reduction in the teacher's perception of conflict in her relationship with the focus child at post intervention $(\mathrm{RCI}=3.35)$; while a more limited increase in closeness was also reported, it did not reach a reliably significant level $(\mathrm{RCI}=.75)$.

The First Step coach and teacher reported the intervention was beneficial. These perceptions were support by outcome data with reliably significant improvements in social skills $(\mathrm{RCI}=13.16)$ and decreases problem behavior $(\mathrm{RCI}=-8.96)$. As well, this child improved his academic engagement from well below normal ranges at baseline (M $=.53, \mathrm{SD}=.12)$ to well above normal ranges at post intervention $(\mathrm{M}=.93, \mathrm{SD}=.00)$.

From the perspective of the logic model, the coach's competent use of MI, strong coach and teacher alliance, and significant change in teacher perception of conflict supported the pathways of influence proposed within the logic model. Unfortunately, the 
FSCCU intervention was not sufficient to allow significant increases in the teacher's rates of positive to negative feedback. This teacher demonstrated more than adequate CLASS component implementation fidelity, which may have influenced this child's responsiveness to the intervention overall.

Child 1173. This child is a six-year-old Caucasian boy, whose teacher is referred to as 5043. According to parent report, child 1173 displayed significant and long-lasting tantrums prior to the intervention. His parents reported being very reluctant to pursue a mental health label, as they are opposed to medication. At school, according to teacher's report, this child was often obstinate, and became overtly sad or angry when asked to comply with adult directions. His teacher also reported significant distractibility that interfered with nearly all aspects of his schooling. This child received no community supports at the time of this intervention. A review of the First Step CLASS monitoring $\log$ indicates this child completed only 13 of the 30 -day intervention cycle, with 10 recycle days on which the child was unable to unable to meet with the behavioral expectations of the intervention, and seven days that the teacher did not implement the intervention or that schedule conflicts did not allow. This resulted in a low intervention dosage (.43) and only 56 percent compliance on the part of the child.

Both teacher and coach rated the coach-teacher alliance as strong; the coach and teacher's alliance ratings were $4.0(\mathrm{SD}=.53)$ and $5.0(\mathrm{SD}=.00)$, respectively. During the intervention, this teacher committed to increasing the amount of positive feedback he used with children in the classroom. The teacher demonstrated a unit rate of positive to negative feedback of 1.26 at baseline, which improved only slightly during the maintenance phase. This teacher was classified as a non-responder (unit rate $=1.70$ ). 
Observations of the limited implementation that did occur indicate excellent intervention adherence (.93) and adequate implementation quality (.84) on the part of the teacher. Calculation of the Reliability Change Index indicated a reliably significant increase in the teacher's perception of conflict in his relationship with the focus child at post intervention $(\mathrm{RCI}=6.7)$, and decreases in closeness that did not reach a reliably significant level $(\mathrm{RCI}=-1.89)$.

The First Step coach reports the child did not respond well to the intervention, and was classified as a non-responder with no reliably significant improvements found in any of the measured child outcomes. In fact, this child's social skills declined $(\mathrm{RCI}=-1.27)$ while his problem behaviors increased $(\mathrm{RCI}=.64)$. Observed academic engagement improved only slightly from baseline $(\mathrm{M}=.36, \mathrm{SD}=.10)$ to post intervention $(\mathrm{M}=.46$, $\mathrm{SD}=.10)$ remaining well below normal ranges.

From the perspective of the logic model, the coach's competent use of MI and strong coach and teacher alliance supported the pathways of influence proposed within the logic model. Unfortunately, the lack of significant change in teacher perception of conflict or closeness, and the teacher's lack of responsiveness to the FSCCU intervention did not. Although this teacher demonstrated more than adequate CLASS component implementation fidelity it was not sufficiently powerful enough to overcome the low intervention dosages, family issues, and possible teacher frustration/fatigue that may have led to poor child outcomes.

Child 1181. This child is an eight-year-old African American boy. According the case report, he had tantrums at home prior to the intervention in which he would become very frustrated and leave the room. Parents also reported he would often become upset 
with his younger brother and sister and refuse to allow them access to toys, or would conscientiously avoid contact with them. In the classroom, this child demonstrated overt social behaviors. For example, there were several instances of inappropriate touching of his peers and instances of frustration often resulted in this child putting his head down on his desk and 'giving up', according to teacher report. According to the coach completed case summary, this child's home life during the intervention was compounded by extreme poverty in a cramped apartment where several families live together. A review of the First Step CLASS Monitoring Log indicated the child completed the 30-day intervention cycle with seven recycle days, and five days on which the intervention was not implemented. This resulted in a low intervention dosage of .60, with only $72 \%$ compliance during those days the intervention was implemented. Significant modifications in the intervention included; the child self-recording his intervention points, identification of other adults in the school who provided additional reinforcement, modified daily intervention intervals, and various reinforcement strategies.

Both teacher $(\mathrm{M}=4.88, \mathrm{SD}=.35)$ and coach $(\mathrm{M}=4.38, \mathrm{SD}=1.06)$ rated the coach-teacher alliance as strong. During the intervention, this teacher committed to improving the amount of positive feedback she used in the classroom as a goal for the program. At baseline, she demonstrated a unit rate of positive to negative feedback of 2.33, improved through the maintenance phase of the intervention and was classified as a Responder (unit rate $=4.42$ ). In addition, her implementation of the First Step CLASS component was classified as adequate for intervention adherence $(.80)$ and excellent for intervention quality (1.00). Calculation of the Reliability Change Index indicates a reliably significant reduction in the teacher's perception of conflict in her relationship 
with the focus child at post intervention $(\mathrm{RCI}=4.46)$; while an increase in closeness was also reported, it did not reach a level of clinical significance $(\mathrm{RCI}=1.89)$.

The First Step coach reports the child responded well to the intervention. Outcomes for this child indicate reliably significant improvements in social skills $(\mathrm{RCI}=$ 5.32). Although problem behaviors were reduced, this reduction did not reach clinical significance $(\mathrm{RCI}=-1.6)$. Observed average academic engagement improved only slightly from baseline $(\mathrm{M}=.50, \mathrm{SD}=.22)$ to post intervention $(\mathrm{M}=.78, \mathrm{SD}=.05)$ remaining slightly below normal ranges.

From the perspective of the logic model, the coach's competent use of MI, strong coach and teacher alliance, and significant change in teacher perception of conflict supported the pathways of influence proposed within the logic model. Additionally, the FSCCU intervention was sufficient to allow significant increases in the teacher's rates of positive to negative feedback. Furthermore, teacher 5030 demonstrated more than adequate CLASS component implementation fidelity, which may have influenced this child's responsiveness to the intervention overall.

Case-level Summary. The coach and teacher alliance was strong across all 12 cases, as was the coaches' adherence to the FSCCU intervention protocol. During the intervention, all teachers set behavioral goals for themselves related to increasing the amount positive and/or decreasing the amount of negative feedback utilized with children in their class. More than two thirds selected increasing the average number of positive feedback statements to children as a goal. The remaining teachers chose to focus on decreasing negative feedback usage, or both. All but one teacher met their goal, and slightly more than half of the teachers met the desired unit ratio for positive to negative 
feedback at post intervention, and were classified as responders to the FSCCU intervention.

On the whole, teachers' First Step CLASS component intervention fidelity was more robust than intervention dosages and compliance proportions. For example, excellent teacher implementation adherence (range $=.87$ to 1.00 ) and adequate teacher implementation quality (range $=.75$ to .93 ) were juxtaposed with moderate intervention dosages (range $=.43$ to .97 ) and compliance (range $=.53$ to .97 ). When First Step CLASS component intervention dosages were above .50, at least one of the three child outcomes was reliably significant. Individual program modifications were present in $\mathbf{5 8}$ percent of the cases, including adjustments to the daily intervention length, individual rewards contingencies, the addition of surrogates to provide additional attention for meeting behavioral expectations, and (in one case) self-recording of intervention points.

Composite-level Analysis. In this section, an analysis of the function of the FSCCU across cases is presented for (a) components of the logic model individually (i.e., across measures) and (b) as a composite including all cases and components of the full logic model. These results are further contextualized using the FSCCU Analysis Classification System (See Table 4, Chapter 3). First, an analysis of outcomes associated with the logic model is presented. Then, to gain a better understanding of the intervention's functioning, the composite level results are presented across the logic model (Figure 2, Chapter 3).

This section begins with the presentation of results from the Coach-Teacher Alliance Survey, followed by the Teacher-Child Relationship Scale, The Observation of Teacher Behavior (changed in the unit ratio of positive to negative feedback), and the 
Enhanced First Step CLASS Fidelity Checklist. Finally, intervention outcomes for children's behavior (i.e., social skills, problem behaviors, academic engagement) are presented. As MI implementation integrity was addressed earlier in this chapter, only the composite level results of this component are presented here.

The Coach-Teacher Alliance Survey was utilized for Wave 2 of Cohort 1 only, and thus results are available for only eight of 12 cases. The composite average coachteacher alliance for the sample (sum of individual composite average scores / sample size) constitutes the analysis of coach-teacher alliances across cases, and is reported next. Teachers' perceptions of the alliance with their coach were higher on average $(M=4.97$, $\mathrm{SD}=.09)$ than were coaches' perception of this alliance $(\mathrm{M}=4.39, \mathrm{SD}=.24)$. The composite scores for coaches and teachers in regards to their individual perceptions of alliance with each other are classified at strong.

Results from Student-Teacher Relationship Scale were used to perform a repeated-measures analysis (equivalent to a paired-t given that the variable is measured only at two time points), to compare the teacher's perception of Conflict and Closeness assessing within-subject change from baseline to post-intervention across cases. There was no significant difference between baseline and post intervention teacher perception of Conflict, $\mathrm{F}(1,11)=1.11, p=.315$. Nor was there a significant difference for teacher perception of Closeness, $\mathrm{F}(1,11)=.73, p=.411$. Although not statistically significant, Student-Teacher Relationship Scale scores trend in the desired direction and produced very small effect sizes for Conflict and Closeness $\left(\eta_{p}{ }^{2}=.021\right.$ and .009 respectively). In total, 77 Observations of Teacher Behavior were completed for a total of 26.5 hours of direct observation of focus teachers in the classrooms. Of this, a total of five and 
one half hours $(21 \%)$ constituted interobserver reliability observations. An agreement ratio for frequency variables was computed for each code category (agreements/[disagreements+agreements] x 100), and the results can be found in Table 8 . The mean agreement for overall praise was $87 \%$ (ranging from $77 \%-94 \%$ ). While the mean agreement for overall reprimands was $84 \%$ (ranging from $58 \%-100 \%$ ). The overall agreement for each code category met a minimal ștandard of $80 \%$ or higher. Agreement scores that dropped below $80 \%$ were analyzed further and found to be due to low occurrences of those behaviors.

Table 8. Interobserver Reliability for Observations of Teacher Behavior.

\begin{tabular}{ll}
\hline Code Category & Percent Agreement (range) \\
\hline Praise Focus child & $91.5(66-100)$ \\
Praise Peer & $79.9(54.2-100)$ \\
Praise Classroom & $95.7(78.5-100)$ \\
Total Praise & $\mathbf{8 6 . 6}(\mathbf{8 0 . 9 - 9 3 . 7 )}$ \\
Reprimand Focus child & $86.6(33.3-100)$ \\
Reprimand Peer & $93.5(86.9-100)$ \\
Reprimand Classroom & $80.7(28.6-100)$ \\
Total Reprimand & $\mathbf{8 3 . 8}(\mathbf{5 8 . 3 - 1 0 0 )}$ \\
\hline
\end{tabular}

Data from the OTB were plotted and reviewed for the purpose of classifying individual cases as responders to the FSCCU based on increases in the total average ratio of praise to reprimands provided overall (to the focus child, any peer, and the class as a whole) by the teacher. In doing so, fair amounts of variability were found, with data that are slightly skewed (standard deviations nearing means) primarily due to low base rates and two extreme cases. Of the Observation of Teacher Behavior categories, only the category of Specific Praise visually demonstrated appropriates for a one-way withinsubject analysis although the analysis was not originally planned for. Table 9 summarizes 
means, standard deviations, and effect sizes for this analysis, including the OTB categories of Total Praise and Reprimand and Total Specific Praise. Data was collapsed, across target-, peer- and classroom-directed feedback, although the general and specific categories for praise and reprimands are displayed. There was a statistically significant increase in the average occurrence of praise $(F(1,11)=10.64, p=.008)$ for this sample of teachers, while the average occurrence of reprimands fell it did not reach statistical significance. Overall teacher behavior change in the category of Specific Praise rose to statistical significance and demonstrated a medium effect size $\left(\eta_{p}{ }^{2}=.220\right)$. For all onesample design (e.g., repeated measures within subjects) analyses, the correlation within subject means has been accounted for and a standard weighted-means analysis was employed. In this case, the partial eta is the same as a partial point-biserial correlation (See Rosenthal \& Rosnow, 2008), and is defined as, "the proportion of total variability attributable to a factor" (J. Seeley, personal communication, January 10, 2012). Thus, its calculation specifically accounts for error variance (SS Treatment/SS Total + SS Treatment Error). Taking the square root of eta-square, and comparing the resulting eta to Cohen's $(1988)$ guidelines $(.14=$ small, $.36=$ medium, and $.51=$ large $)$ we find the significance of an eta-square of $.220($ eta $=.47$ ) to be considered a medium effect size.

Table 9. Within-subjects Analysis; Observation of Teacher Behavior categories.

\begin{tabular}{llllll}
\hline & Baseline & Post & $F$ & $p$-value & $\eta_{\mathrm{p}}{ }^{2}$ \\
\hline Total & & & & & \\
$\quad$ Praise & $36.3(24.3)$ & $62.3(23.9)$ & $10.64^{*}$ & .008 & .192 \\
$\quad$ Reprimands & $29.5(26.6)$ & $19(11.6)$ & 2.2 & .166 & .050 \\
$\begin{array}{l}\text { Specific } \\
\quad \text { Praise }\end{array}$ & $11.3(9.6)$ & $24.5(12.0)$ & $10.21^{*}$ & .008 & .220 \\
\hline
\end{tabular}

*Indicates $\mathrm{p}<.05$ 
Adherence and quality scores were calculated for teacher implementation of the First Step CLASS component. Adherence scores ranged from .87 to 1.0, with a mean average across cases of $.95(S D=.04)$. Quality scores ranged from .68 to .93 , with a mean average across cases of $.83(\mathrm{SD}=.07)$. Overall adherence and quality ratings were quite high, with overall teacher adherence scores classified as excellent and overall teacher quality scores classified as adequate.

Table 11 summarizes the aggregated standardized scores for teacher-reported social skills, problem behavior, and academic engagement. Standard scores from the SSiS for teacher perception of children's social skills at baseline ranged from 64 to 102 with a mean value across cases of $78.25(\mathrm{SD}=11.06)$; at post-intervention scores ranged from 64 to 128 with a mean value across cases of $92.08(S D=19.96)$. Standard scores for Problem Behaviors at baseline ranged from 114 to 154 with a mean value across cases of $133(\mathrm{SD}=13.33)$; at post-intervention scores ranged from 93 to 157 with a mean value across cases of $122(\mathrm{SD}=19.21)$. Children's observed percentages of AET at baseline ranged from .36 to .87 with a mean value across cases of $.67(\mathrm{SD}=.17)$, and a postintervention ranged from .47 to .89 with a mean value across cases of $.79(\mathrm{SD}=.12)$. 
Table 10. Child Intervention Outcomes.

\begin{tabular}{|c|c|c|c|c|c|c|c|c|c|}
\hline \multirow[t]{2}{*}{$\begin{array}{l}\text { Child } \\
\text { ID }\end{array}$} & \multicolumn{3}{|c|}{$\begin{array}{l}\text { Social } \\
\text { Skills } \\
\end{array}$} & \multicolumn{3}{|c|}{$\begin{array}{l}\text { Problem } \\
\text { Behavior }\end{array}$} & \multicolumn{2}{|c|}{$\begin{array}{c}\text { Percentage } \\
\text { AET }\end{array}$} & \multirow[t]{2}{*}{$\begin{array}{c}\text { Classification } \\
\text { System }\end{array}$} \\
\hline & $\begin{array}{l}\text { Base } \\
\text { (SS) }\end{array}$ & $\begin{array}{l}\text { Post } \\
\text { (SS) }\end{array}$ & $\mathrm{RCI}$ & $\begin{array}{l}\text { Base } \\
\text { (SS) }\end{array}$ & $\begin{array}{l}\text { Post } \\
\text { (SS) } \\
\end{array}$ & $\mathrm{RCI}$ & $\begin{array}{l}\text { Base } \\
\text { (SD) }\end{array}$ & Post (SD) & \\
\hline 1100 & 88 & 113 & 9.87 & 133 & 105 & -8.64 & $.86(.05)$ & $.89(.12)$ & Responder \\
\hline 1106 & 102 & 128 & 10.12 & 122 & 112 & -2.88 & $.81(.08)$ & $.80(.15)$ & Responder \\
\hline 1109 & 68 & 86 & 7.34 & 130 & 118 & -3.52 & $.84(.11)$ & $.86(.08)$ & Responder \\
\hline 1117 & 80 & 99 & 7.59 & 151 & 110 & -12.48 & $.60(.16)$ & $.81(.06)$ & Responder \\
\hline 1123 & 70 & 68 & -0.51 & 150 & 157 & 1.6 & $.54(.14)$ & $.82(.14)$ & Non \\
\hline 1128 & 67 & 86 & 7.59 & 141 & 123 & -5.76 & $.61(.25)$ & $.88(.04)$ & Responder \\
\hline 1144 & 81 & 91 & 4.05 & 119 & 121 & 0.32 & $.66(.17)$ & .68( & Partial \\
\hline 1163 & 64 & 64 & 0.25 & 154 & 155 & 0.32 & $.87(.09)$ & $.80(.01)$ & Non \\
\hline 1164 & 88 & 94 & 2.28 & 126 & 126 & 0 & $.84(.01)$ & $.82(.07)$ & Partial \\
\hline 1170 & 81 & 115 & 13.16 & 123 & 93 & -8.96 & $.53(.12)$ & $.93(.00)$ & Responder \\
\hline 1173 & 70 & 67 & -1.27 & 133 & 135 & 0.64 & $.36(.10)$ & $.47(.10)$ & Non \\
\hline 1181 & 80 & 94 & 5.32 & 114 & 109 & -1.6 & $.50(.22)$ & $.78(.05)$ & Partial \\
\hline
\end{tabular}

SSIS Social Skills, Problem Behaviors, SSBD Academic Engaged Time. SS = Standard Score; Bold $=$ RCI statistic $>1.96$ or $>-1.96$; Bold Percentage AET $=$ normal range per the SSBD manual.

Results from SSiS were used to perform a one-way within-subject analysis, to compare the teacher's perception of Social Skills and Problem Behaviors assessing within-subject change from baseline to post-intervention across cases. There was a significant effect on teacher perception of Social Skills at post-intervention, $F(1,11)=$ $16.28, p=.02$, and Problem Behaviors, $\mathrm{F}(1,11),=6.23, p=.029$. A similar analysis demonstrated a significant effect on observed percentages of AET at post-intervention, $F$ $(1,11)=8.16, p=.016$. 
Table 11. Within-subjects Analysis; Social Skills and Problem Behaviors.

\begin{tabular}{llllll}
\hline & Baseline & Post & $F$ & $p$-value & $\eta_{\mathrm{p}}{ }^{2}$ \\
\hline $\begin{array}{l}\text { Social Skills } \\
\text { Problem }\end{array}$ & $78.25(11.06)$ & $92.08(19.96)$ & 16.28 & $.002^{*}$ & .150 \\
$\begin{array}{l}\text { Behaviors } \\
\text { Academic }\end{array}$ & $133(13.33)$ & $122(19.21)$ & 6.23 & $.029^{*}$ & .091 \\
Engagement & $.67(.17)$ & $.79(.12)$ & 8.16 & $.016^{*}$ & .136 \\
\hline
\end{tabular}

Composite Logic Model Analysis. Provided next is Figure 3, the logic model diagram. Composite results are represented within each element.

Figure 3. Logic Model Composite.

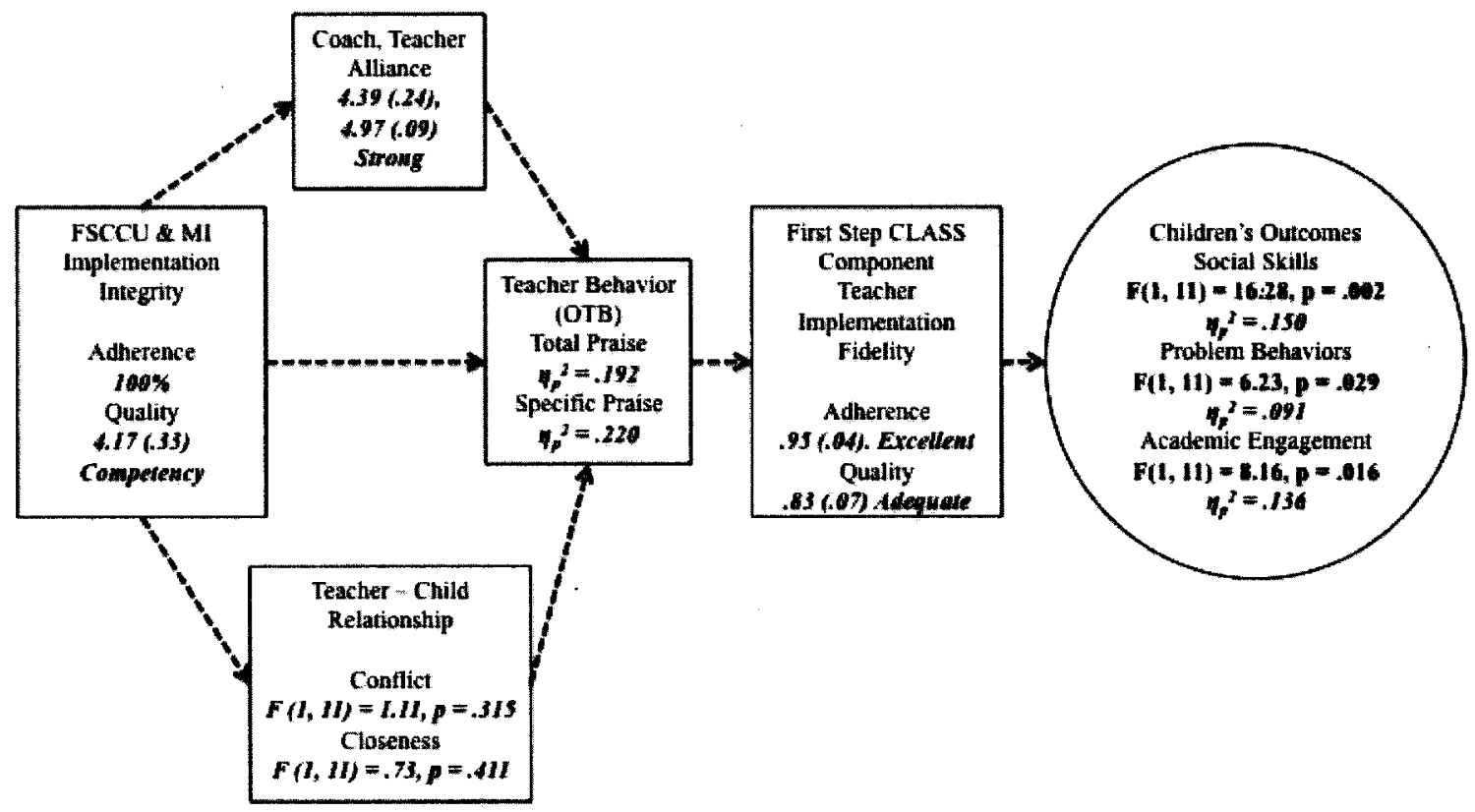

When viewed from the perspective of the logic model, these evaluations shed

light on the hypothesized functioning of individual components within and in relation to the FSCCU intervention. The FSCCU implementation integrity was categorized as excellent for adherence, and competent in terms of MI quality. First Step coaches implemented each step in the FSCCU intervention protocol, and demonstrated 
competency in their application of MI. This finding was based on an independent coding of 12 audio-recorded interactions between coaches and subject teachers utilizing a widely accepted tool for the rating of MI proficiency, the MITI. Coach and teacher alliances were strong, with coaches' perceptions only slightly lower than those of the teachers. Overall, teachers' perceptions of the teacher-child relationship did not change to a statistically significant amount for either the Conflict or Closeness subscales. Although both subscale scores demonstrated positive trends, with five teachers perceiving reliably relevant decreases in Conflict, while only one perceived reliably relevant increases in Closeness at post-intervention. Teachers changed their feedback behavior in the classroom from baseline to post-intervention as demonstrated by the Observation of Teacher Behavior system. Significant results were obtained from a within-subject analysis of the OTB categories, specifically the average occurrence of praise showed statistically significant improvement $\left(\eta_{\mathrm{p}}^{2}=.192\right)$. Upon further analysis, this finding resulted from the category of Specific Praise, which rose to statistical significance and demonstrated a medium effect size $\left(\eta_{\mathrm{p}}^{2}=.220\right)$. Additional positive findings came from teachers' adherence to the First Step CLASS component of the intervention. Ratings from observations of their implementation of this intervention were categorized as Excellent. As well, observations of the quality with which teachers implemented the CLASS component with focus children in their classrooms was categorized as adequate. Outcomes for the focus children who participated were strong overall. Statistically significant effects on teacher perception of Social Skills, Problem Behavior and children's observed academic engagement at post-intervention were present. 
Overall, evidence indicates the logic model may function as hypothesized for all but the teacher-child relationship, as no statistically significant evidence was found across cases that indicate teachers' perceptions of the teacher-child relationship are malleable. These results may provide limited evidence that the teacher-child relationship acts as moderator within this logic model, not as it is currently displayed as a mediator between the integrity with which MI is implemented and teacher behavior. However, this composite finding for the teacher-child relationship is juxtaposed with case-level indications that statistically reliable positive changes did occur in teacher perceptions of their relationship with the focus child in six cases ( 5 for Conflict, and 1 for Closeness). 


\section{CHAPTER V}

\section{Discussion}

This dissertation examined enhancements to the First Step to Success early intervention program developed by infusing MI (Miller \& Rollnick, 2002) procedures into the existing protocol. The resulting FSCCU, an adaptation of Reinke et al.'s (2008) Classroom Check-up, focuses on the classroom teacher's use of praise to help replace the systematic use of external reinforcers; and to reduce the negative attention for inappropriate behavior (reprimands), which inadvertently maintains the challenging behavior teachers seek to eliminate. The FSCCU was explored through an open multiplecase-study design (Meyers, et al., 2007) to investigate implementation fidelity, the intervention components' social validity, and the extent to which the logic model functioned as intended.

This study advances existing knowledge and makes unique contributions in several areas. First, it extends nearly two decades of work examining the original First Step to Success program by systematically examining enhancements designed to substantially decrease problematic behavior and increase adaptive behavior of the most severely disordered students (Carter \& Horner, 2007, 2009; Diken \& Rutherford, 2005; Golly et al., 2000; Golly, Stiller, \& Walker, 1998; Nelson et al., 2009; Overton et al., 2002; Sprague \& Perkins, 2009; Walker et al., 2005; Walker et al., 1998; Walker et al., 2009). 
Second, this study attends to the development of the intervention and its future by enlisting the perspectives of those involved in it to examine the social significance of intervention goals, the appropriateness of procedures, and the importance of outcomes (Frey, et al., 1991). The findings suggest the enhancements are socially valid and promising.

Third, this study extends the literature base related to the application of MI in school settings. While a number of studies have investigated interventions that infuse MI into their procedures, the extent to which interventionists in school settings actually implement MI with fidelity has not yet been addressed; this study is the first examination of the MI fidelity, proficiency, and quality of interventionists within a school-based application. The results are important as they suggest this is a feasible approach within the schools, and that the training and supervision procedures utilized with coaches were successful. Furthermore, the findings lend support to the possibility that school personnel can learn to implement MI with some level of acumen, and reveal complications with the functions of a tool commonly used to measure MI implementation proficiency (the MITI) in school-based applications.

Fourth, this study represents the first attempt to measure the teacher-child relationship within the First Step to Success intervention. This is particularly relevant given the needs of the population of children who were identified for this study, and the extant literature pertaining to the debilitating nature of coercive home and school transactions. The results of this exploration - that the teacher-child relationships may be malleable - were inconclusive; these relationships (overall) did not demonstrate 
statistically significant improvement, yet case-level analyses indicate statistically reliable positive trends in half of the sample.

Overall, study provides preliminary evidence that the FSCCU can be implemented with fidelity, is socially valid, that the logic model functions as intended. This chapter examines the implications of the findings in these areas, and concludes with a discussion of the limitations of this study and the implications for future educational research, and practice. Next, a discussion of the findings for each research question is presented.

\section{Implementation Fidelity}

Coach reported-Classroom Check-up process fidelity and independently rated audio recordings of coach-teacher interviews using a modified version of the MITI code were used to address adherence to the intervention process and quality of MI implementation. The results from both measures suggest the FSCCU was implemented with acceptable adherence and quality- two important aspects of intervention fidelity (Fixsen, et al., 2005; Gresham, 1989; Sanetti, et al., 2009).

With regard to adherence, coaches self-reported excellent consistency in completing the four steps of the FSCCU intervention. Although the Classroom Check-up Process Fidelity Checklist was designed as a post measure, our coaches utilized it as a guide prior to and during teacher interviews. Checklists such as this have proven useful in situations of complexity, when used as a pre, concurrent, or post guide to increase the fidelity of implementation (Gawande, 2009). In addition, the average length of the FSCCU teacher interviews was only 32 minutes. As time is often a barrier for teachers, 
the brief nature of this intervention may support its appropriateness for application in authentic educational settings.

Procedures within the FSCCU have been very carefully crafted to support coach implementation of not only the global MI spirit, but also specific MI skills, and this appears to be time well spent. According to an independent evaluation, coaches were able to meet most proficiency standards for MI practice. Specifically, all three coaches met the Competency thresholds for global ratings (i.e., collaboration, autonomy and choice; directing clients towards change; understanding; reflection; and evocation). Additionally, MITI summary scores, which are based on specific behaviors counts to judge the proficiency with which a coach utilizes MI, ranged from Competency to Proficiency across the seven indicators (i.e., open ended questions, close ended questions, simple reflections, complex reflections, $\mathrm{MI}$ adherent statements, MI non-adherent statements, and information giving). These results are a testament to the rigorous training and coaching procedures utilized in the early stages of the intervention's development, and bodes well for the likelihood of the intervention going to scale (i.e., external validity) (Sanetti, et al., 2009).

\section{Social Validity}

Questionnaires completed by First Step coaches and teachers, and focus group interviews for teachers, suggest the FSCCU intervention is socially valid. Social validity is an important aspect of any intervention, and plays a major role in the likelihood that the intervention will be implemented and successful when deployed in schools (Carr, et al., 1999; Conroy, et al., 2005; Frey, et al., 2010; Hieneman \& Dunlap, 2000; Hieneman, 
et al., 2005; Lane \& Beebe-Frakenberger, 2004; Papalia-Berardi \& Hall, 2007; Schwartz \& Baer, 1991).

Data from Social Validity Questionnaires indicate strong levels of satisfaction with the FSCCU intervention for both teachers and coaches. Teachers, in particular, indicated extremely high satisfaction. The lack of variability in the teachers' responses to satisfaction questions makes it difficult to tease out any reasonable conclusions, other than teachers agreed the goals of the FSCCU intervention are important, the procedures are acceptable, and the outcomes favorable. Coaches also responded favorably to social validity questionnaires. Specifically, coaches agreed that the intervention was compatible with the needs of the teachers, effective in teaching new strategies, and had positive effects on teacher-child interactions.

Additional evidence that this sample of teachers found the FSCCU procedures to be socially valid arose from the analysis of focus group interviews completed at post intervention. Unlike the questionnaires, much variability was evident in teachers' perceptions of the importance of positive attention as a means of classroom management. The following findings were particularly salient.

First, the majority of teachers felt as though the focus on positive attention as a foundation to the FSCCU intervention was important and worked to benefit children in many aspects of their school adjustment and behavior. Other teachers, of children in selfcontained settings with severe emotional and behavioral disorders, were more cautious. They feared these children's ability to return to typical classrooms might be hampered if work did not ensue to "fade positive attention... to the level of a regular classroom." Two teachers struggled with the use of positive attention for behaviors they expected children 
should know and be able to perform. As these differing teacher perspectives were analyzed via a constructivist, thematic (Braun \& Clarke, 2006) approach, a theme of balance emerged from the teacher's own words. Many teachers from differing perspectives on the importance of positive attention used "balance" to describe what they felt was the appropriate application of positive and negative attention (i.e., praise and reprimands). However, the importance that this sample of teachers placed on the use of positive and negative attention as a means of classroom and behavior management was evident in their responses to focus group question prompts. Individual teacher's circumstance (e.g., self-contained classrooms) and experience (e.g., with behaviors teachers expect children to know and be able to demonstrate) seemed to have influenced differing perspectives in regards to the application positive and negative attention in classrooms. These differing perspectives might best be served with an emphasis on the concept of balance, which was expressed by many teachers, and is addressed within the universal principles that serve as the intervention's foundation.

The majority of teachers agreed that the FSCCU intervention was not a burden on their time or in the complexity of tasks that were required. This finding supports those of Reinke; whose original research found that reduced burden for teachers was an important benefit of self-monitored visual performance feedback (Reinke, et al., 2007; Reinke et al., 2008). Reinke found that most teachers were not burdened by one feedback and goal development session and daily observation and feedback routines that capitalized on the teachers' self-monitoring of the data, although two teachers found the daily observation schedule slightly intrusive. The FSCCU reduced this burden even further, utilizing a three-hour training session, one feedback and goal development session; and only four 
observation and feedback routines that also capitalized on teacher self-monitoring routines; none of the teachers commented on intrusiveness. Apparently, the limited time burden and fewer observation and feedback routines were acceptable for this sample of teachers.

During the focus group interviews teachers were asked about perceptions of their own motivation, specifically: To what extent were you motivated towards change after the feedback session and review of observational data? Teachers volunteered mostly positive feedback in regards to their interpretations of the data from the Observations of Teacher Behavior. Some teachers were surprised and felt they could do better; others were encouraged by the results and chose to concentrate on specific behavioral feedback. Teachers appreciated the simplicity with which the data from the Observation of Teacher Behavior was presented. Several teachers commented on the overall meaningfulness of the data presented to them. Nearly all appreciated the tone with which the data was presented, which could be interpreted as teacher's appreciation for the global spirit of MI (i.e., collaboration, autonomy and choice; directing clients towards change; understanding; reflection; and evocation), an area found to be a relative strength in our coach's application of MI skills. Furthermore, these teachers' had linked their motivation to change to data based interpretations of their own behavior from data provided during the FSCCU intervention. Asking teachers to link a central tenet of MI - their own motivation for change to the intervention - represents an extension to the visual performance feedback research of Reinke et al., (2007) and the original Classroom Check-up of Reinke et al. (2008), and adds support to the hypothesized effect of the intervention on the motivation of teachers. 
The focus group interviews were also successful in evoking teachers' perceptions of weakness in the FSCCU intervention procedures, and how the intervention might be improved. During these interviews at least two teachers responded to the values discovery interview unfavorably, in that the questions utilized were too philosophical and could be addressed more straightforwardly. Many teachers did not remember or recognize the universal principles. Additionally, several teachers could not recall their personal goals developed during the Data Review and Goal Setting step. This is concerning and speaks to the need to bolster the Goal Setting process.

In summary, teachers were satisfied with the FSCCU intervention and responses to the social validity questionnaire in this regard were consistently high. The lack of variability as a result of consistently favorable responses, on these questionnaires, limited the analysis of the results. The strength of the social validity data is evident particularly in regard to triangulation. Specifically, the convergence of similar information from multiple methods (qualitative and quantitative) and sources (coach and teachers) provides a compelling case for the social validity of the FSCCU Intervention. Social validity is particularly important in the early stages of development because attention to this factor increases the likelihood it will be adopted and implemented with fidelity, which are critical factors for scale up efforts (Frey, et al., 2010).

\section{Functionality}

Evidence strongly suggests that the FSCCU intervention functioned as was proposed in the logic model. However, the current research design does not allow for a causal interpretation of these findings. In the following sections, interpretations of the 
data for the areas of alliance, teacher-child relationship, and teacher behavior, and child outcomes are presented in the context of the logic model's components.

Alliance. Results from the Coach-Teacher Alliance Survey strongly suggest that coaches and teachers perceived a high quality relationship. Interestingly, teachers' perception of their alliance with coaches was stronger than the coaches' perceptions of their relationship with teachers across all questions on the survey. For example, coaches did not feel the teachers communicated as effectively as the teacher's felt that the coaches did. As well, coaches felt the time spent with teachers was not as effective and productive as teachers felt it was. This is understandable as teachers have numerous responsibilities that draw on their time, and capacity for communication and productivity, while coaches' responsibilities are more limited. Although limited variability exists in this data, it certainly provides preliminary evidence that the coach-teacher relationship was strong. This is important during the application of MI, as the significance of client engagement in relation to intrinsic motivation and influence is a foundational element in Client Centered Therapies (Rogers, 1951; 1959), and is apparent in the "spirit" of MI that works to build autonomy, competence, and relatedness (Deci \& Ryan, 2000) in the client. Although the design does not permit definitive statements about causality to be made, the social validity data suggests the global spirit of MI (i.e., collaboration, autonomy and choice; directing clients towards change; understanding; reflection; and evocation) and/or the client-centered approach of the four steps of the FSCCU were contributing factors to the quality of this relationship. Throughout the focus group interviews, teachers' comments in regards to their coaches were overwhelmingly positive and exemplified the global spirit of MI. As an example, two teachers experiences are provided below for one 
particular element of the global spirit of MI, from their own words. One teacher, at an elementary school designed as a self-contained unit for children with severe emotional and behavioral disorders, offered the following in response to the question "What did you enjoy most about the FSCCU".

It was great to have someone other than a principal or Kentucky Teacher Internship Program [representative] to come in without an agenda to provide pure feedback on what I was doing - you know she did not care if I passed or failed I mean she did not have an agenda - she was not trying to get a certain ratio for the superintendents office or anything like that she was just coming in doing pure data and I liked that.

This teacher represents her experience of the coach's neutrality, a skill found to be of significant benefit during MI (Miller, 2012; Wagner, 2012), and is often used to instill a sense of autonomy in the client. Another teacher from a more typical elementary school commented on the autonomy she felt from the coach's interactions with her.

So he kinda left it up to you to decide on what the goal was based on. The material or the feedback he gave. He showed me what - like "here is what I observed" and then said "what do you think of it?"

These examples represent expressions of support for the coach-teacher relationship, and in the context of the intervention represent not only the coaches' proficient use of MI, but the honest responses from the teachers. These, as well as the positive results from the Social Validity Questionnaire, lend support to the possibility that during the FSCCU intervention coaches' worked to develop a strong alliance with the teacher. Motivational Interviewing is heavily reliant on a working alliance (i.e., rapport 
or therapeutic relationship) to create safe and understanding environment for change (Miller \& Rollnick, 2002). As an extension to the current research on school-based applications of MI (Dishion, Stormshak, \& Siler, 2010; Frey et al., 2011; Reinke et al., 2008) the reflections of these teachers lends support to the importance of the working alliance in this context.

Teacher-Child Relationship. Previous First Step research had not systematically studied teacher-child relationships. Substantial research suggests these relationships (Hamre and Pianta, 2001; Jerome, Hamre, \& Pianta, 2009; Ladd \& Burgess, 1999; see also Rudasill, 2011) may be difficult to change. If the teacher's perception of the relationship is intractable, conflictual teacher-child relationships may act to moderate rather than mediate the FSCCU intervention. If accurate, there would be important, and somewhat discouraging, implications for interventionists desperate to improve teacherchild relationships. Conflict has demonstrated more robust association with academic and adaptive school outcomes than children's problem behavior (Baker, 2006; Hamre and Pianta, 2001). Ladd, Birch, and Buhs (1999) found that teacher-child conflict was directly associated with lower levels of classroom participation than was closeness. The current study represents the first attempt to measure this relationship within the First Step to Success intervention and to explore the likelihood that this relationship may change with the implementation of the intervention.

Overall, changes in teachers' perceptions of the teacher-child relationship did not reach statistical significance for either the Conflict or Closeness subscales. However, both subscale scores demonstrated positive trends, with five teachers perceiving reliably relevant decreases in Conflict, and one perceived reliably relevant increases in Closeness 
at post-intervention. This change, from baseline to post intervention, was more dramatic for Conflict than for Closeness. A case-level exploration of teacher-child relationships within the First Step to Success intervention was more positive, and indicates that teacher perception of this relationship may be malleable in some situations. This is not surprising, as the First Step to Success program targets children with developing externalizing problem behavior (Walker et al., 1997), and that the intervention (in its entirety including the FSCCU) resulted in appreciable reductions in problem behaviors, which are conflictual in nature (see Walker, Colvin, \& Ramsey, 1995). Whereas the First Step to Success program also positively affects the social skill development of focus children, these are often peer related social skills and perhaps more likely to affect peer-peer relationships, not those associated with the teacher-child relationship. Thus it is reasonable to assume that the Conflict subscale of the STRS would be more sensitive to changes in the teachers' perception of their relationship with focus children within the First Step to Success intervention.

The data provides some evidence that the intervention may be associated with improved teacher-child relationships. It is also plausible that the intensity of a teacher's perception of their relationship with a focus child at baseline influences this variable's effect within the logic model, particularly when conflict is initially high. For example, a teacher with very high ratings of Conflict, and low ratings of Closeness may have formed an intractable relationship perception; comparatively, a teacher with more moderate perceptions of Conflict and Closeness may be less entrenched and thus more open to change. From a transactional perspective (Sameroff \& Fiese, 2000; Sameroff \& 
MacKenzie, 2003), the intensity of the teacher's perceptions should be considered, as it interacts with the severity of the child's needs.

Teacher Behavior. After implementation of the FSCCU intervention, each teacher in our sample was motivated to set a personal goal, and observations indicate that each teacher changed their behavior in positive ways (either increased praise and/or decreased reprimands). This change compliments the behaviorally oriented First Step CLASS component; as reinforcers are faded towards the end of the intervention; ideally, the teacher's use of praise replaces the systematic use of external reinforcers upon which the original intervention is based. This is particularly important in classrooms where coercive interactions exist that are often the result of excessive reprimands, and low unit rates of praise to reprimands (Jenson, et al., 2004; Maag, 2001; Nicholas, Olympia \& Jenson, 2001; Shores, Gunter, \& Jack, 1993; Sutherland \& Oswald, 2005; Sutherland, Wehby \& Copeland, 2000). Interestingly, the case-level analysis demonstrated that three teachers (25\%) began the intervention process with unit rates of positive to negative feedback at or above the desired rate of 2.9 (Frederickson \& Losada, 2005, see also Sprague \& Perkins, 2009); countering the premise that excessive rates of reprimand and low rates of praise to reprimand often exist in these classrooms. Even so, two of these teachers increased their unit rates throughout the intervention and were classified as responders; the focus children in their classrooms were also classified as responders. One of these three teachers fell just below the desired unit rate at post-intervention and was classified as a non-responder, as was the focus children in this classroom. The nine remaining teachers $(75 \%)$, those who began the intervention process at baseline with unit rates of positive to negative feedback below the desired rate, improved their unit rates 
throughout the intervention. Five (42\%) of these teachers exceeded the desired unit rate at post-intervention and were classified as responders, as were four of the five focus children in their classrooms (classified as partial responders or responders based on child outcome measure). In total, seven teachers $(58 \%)$ met or exceeded the desired unit rate at post intervention. Again, the design of this study is not sufficient to attribute the change in teacher behavior to the FSCCU alone. However, the association between these three (intervention, teacher behavior, child outcome) provides some evidence that the workings of the proposed logic model may be tenable.

Three categories of change, which were consistent across the 12 teachers who participated, have been identified. The first category is comprised of those teachers who increased their average use of praise and decreased their average use of reprimands. This category accounted for six teachers, and of these six, five teachers met the targeted unit rate (2.9) of praise to reprimands and were categorized as Responders to the intervention. A second category is comprised of teachers who increased their average use of both praise and reprimands. This category accounted for three teachers, and of these three; two met the targeted unit rate of praise to reprimands and were categorized as Responders to the intervention. Finally, three teachers comprised the third category of change in which teachers decreased their average use of both praise and reprimands. Of these three teachers, only one met the targeted unit rate of praise to reprimands and was categorized as a Responder to the intervention. Categorizing teachers' responses to the intervention in this way raises a number of interesting questions in regards to the teachers' personal goals. One might suspect that teachers' behavior change is related to the personal goals each teacher set during the Data Review and Goals Setting step of the intervention. Three 
categories of teacher goals were identified from a review of the coach completed FSCCU Action Plans. Of the 12 teachers, nine teachers chose to increase their use of praise, two chose the decrease of reprimands, and only one chose a combination goal, which identified increased praise and decreased reprimands. Unfortunately, teacher's personal goals, overall, were not obtained. For example, the category of teachers whose use of praise and reprimands decreased over the course of the maintenance observations $(\mathrm{n}=3)$ each had set a goal of increased use of praise. As well, a majority of teachers who participated in focus group interviews at post-intervention revealed they could not recall their specific goals, which may be further evidence of a disconnect between personal goals and behavior change. The majority of teachers whose resulting change included both increased rates of praise and decreased rates of reprimands met the target unit rate objective of 2.9 , as did the majority of teachers whose resulting change included increased rates of praise and reprimands. However, the majority of those teachers whose resulting change included decreased rates of praise and reprimand did not meet the target objective.

\section{CLASS Component Implementation Fidelity. First Step CLASS component} teacher implementation fidelity is recognized in previous research as an important mediator (See Walker et al., 2009). Thus, the fidelity with which teachers implemented the First Step CLASS component appears within the proposed logic model to explore any influence that may result from the teacher's participation in the FSCCU intervention. The resulting teacher implementation fidelity of the CLASS component was strong as indicated by excellent adherence to the CLASS component protocols, and adequate implementation quality. These results are comparable to previous First Step research 
outcomes for implementation adherence and better than previous findings for implementation quality (See Walker et al., 2009).

Moreover, a review of the First Step Classroom Monitoring Logs, completed by subject teachers, and subsequent calculation and analysis reveals a positive correlational relationship between intervention dosage and student compliance. In fact, the Pearson Correlation Coefficient was calculated at $.92(t=6.93, p=<.001)$. This strong positive relationship suggests these factors are somehow related. It may be that children who participate in more intervention program days benefit from the intervention, and are likely to have increased compliance. It may also be that children who are highly compliant with the intervention are provided more intervention program days. Previous research of the First Step to Success program also indicates the importance of intervention dosage (Seeley, et al., 2009; Sprague \& Perkins, 2009).

Child Outcomes. Consistent with previous research findings (see Walker, et al., 2005), children identified for this study demonstrated significantly improved social skills, problem behaviors, and academic engagement. Overall, three fourths of the focus children were classified as Responders or Partial Responders to the intervention. Child outcomes were favorable, and at least anecdotally appear related to successful implementation and completion of the intervention. For example, when exploring the results of individual measures as a composite across the logic model, we find several interesting associations between teacher-child relationships, teacher behavior, intervention dosage and student compliance, and child outcomes. First, given the positive correlation between intervention dosage and student compliance it would be logical to assume that children who were categorized as responders to the program based on 
significant increases in social skills and academic engagement, and decreases in problem behavior may have higher rates of intervention dosage or had higher rates of compliance with the program than did children who did not respond to the program. However, this is not the case. The average intervention dosage and compliance for responders $(M=.63$, and $M=.60$ respectively) was essentially the same as for non-responders $(M=.65$, and $M$ $=.68$ ). Thus, intervention dosage and student compliance does not appear to be the only factor responsible for the differing responsiveness to the intervention found between Responders and Non-responders.

Consistent with the findings from Reinke et al. (2008), these teachers' behavior does seem to be a contributing factor to children's overall responsiveness. Of the nine children who were categorized as Responders or Partial Responders, four had teachers who were categorized as Responders for changes in teacher behavior. Where as, the four remaining Responders or Partial Responders were children who had teachers categorized as Responders for changes in the teacher-child relationship. One child's teacher was categorized as a responder for both changes in behavior and perception of relationship. Although the current research design does not allow for analysis of moderation and mediation effects, the anecdotal evidence lends credence to the importance of changes in teacher behavior, and teacher-child relationships in relation to positive child outcomes.

\section{Summary}

Overall, the three First Step coaches who implemented the FSCCU intervention did so with excellent adherence to the intervention protocol, and proficient ratings suggest coaches applied MI with high quality. While coaches' perception of their alliance with teachers was slightly less robust than teachers' perceptions of their alliance with 
coaches; the quality of the coach-teacher alliances was strong across all cases. Proficient coach implementation of the FSCCU (i.e., adherence and quality), and highly rated coach-teacher alliances suggest these elements are important to the function of the logic model and possibly the previously established associations between teacher implementation and child outcomes (Walker et al., 2009).

The teachers' relationships with focus children over the course of the intervention appear to improve more so for Conflict than for Closeness. This is logical given that the First Step intervention is known to decrease problem behaviors in the focus child, a likely contributor to conflictual teacher-child relationships; it is significant given "relational negativity is more robustly associated with children's outcomes than the degree of closeness in the existing literature" (Baker, 2006, p. 213). Further evidence of the potency of conflictual teacher-child relationships can be found in the coercive models of teacher-child interaction described earlier (McEvoy \& Welker, 2000; Patterson, Reid, \& Dishion, 1992; Reinke \& Herman, 2002), and that our sample were predominantly male (Rudasill, 2011; Rudasill, Reio, Stipanovic, \& Taylor, 2010; Rudasill \& Rimm-Kaufman, 2009). After the implementation of the FSCCU intervention teachers were motivated to set personal goals to support efforts to change their classroom use of praise and reprimands. Unfortunately, these goals did not correspond to teacher outcomes, and teachers had difficulty recalling their personal goals during post-intervention focus group interviews. Thus, goal setting did not appear to have had the effect of goal attainment. In spite of this, goal setting may still be a useful procedure, as teacher behavior in relation to the use of praise and reprimands did change after the implementation of the FSCCU intervention. In particular, a majority of teachers increased their personal rates of praise 
while also decreasing their personal rates of reprimand to a level which resulted in a unit rate of praise to reprimands of 2.9 or higher (the target objective for the study). Teachers implemented the First Step CLASS component with fidelity, which was a likely contributor to significant increases in social skills, problem behaviors, and AET. In spite of the severity of these children's needs, the outcomes for teacher implementation fidelity and child outcome are similar to the positive findings from previous research on the First Step to Success Program. Although comparisons of the effects are not appropriate, these findings are encouraging given that the program has been less effective with more severely disabled children.

\section{Limitations}

The results from this study are promising, yet important limitations exist. The non-experimental design utilized for this study does not control for threats to internal validity. The most significant threats are history and statistical regression. Much of the observational data gathered for this study was the responsibility of the behavior coaches who worked with the subject teachers and focus children. Inter-rater reliability, calculated as percentage of agreement, was strong for the observation measures utilized in this study. History is a serious threat within the case level analysis, but fairly well controlled overall through replication across subjects, multiple sites, and two waves of implementation. The most significant threat is regression. Because children were identified based on low social skills and high problem behaviors, it is possible that focus children's scores would have improved, or regressed towards the mean, even if no intervention was implemented.

Threats to external validity exist as well. Threats to the external validity of this research arise from elements of the research design that do not control for individual 
differences, geographical differences, and/or differences in either due to chronological issues. First, only two school districts were included in the study, both located in the same part of the country. Although our screening procedures appear to have functioned effectively, schools were selected based on their initial receptivity. The schools may not be representative of schools nationally or locally. Second, this sample of teachers may not be representative of K-3 teachers nationally, or within these school districts for that matter. There may be differences in teachers who did and did not participate; it is possible we received those that needed this intervention the least (or the most), and were likely to be the most (or the least) receptive to this type of support. Additionally, the students that participated in this project may not be representative of K-3 children with the most significant behavior problems nationally or within these school districts. Other teachers of and children with severe behavior disorders may indeed not look, act, or respond in a similar fashion if this study was to be replicated.

High satisfaction, social validity outcomes, and fidelity results may be indicative of a second threat to the external validity of this research; the social pressures in the research context that can lead to posttest differences that are not directly caused by the treatment itself. Specifically, there may be demand characteristics- subtle, unprogrammed cues that communicate to subjects something about how they should behave- placed on teachers and children. Although not intentional, it is likely that the teachers and children knew what we wanted and gave it to us. This is exacerbated by the fact that our coaches had strong working alliances, making it reasonable to assume some demand characteristics were influential. For example, respondents may have been influenced by Hawthorne Effects (Suter, 2006) while completing paperwork with their 
coach, children's behavior may have been influenced by the presence of the coach, or trained observers may have been unconsciously influenced by the goals of the project. Furthermore, coaches served as research staff, and to some extent participants in that their self-reports of protocol adherence were utilized. It is very possible they experienced demand characteristics, as the success of the intervention had fairly large implications for them professionally. Replication efforts and future studies should employ an experimental design, and a larger randomly selected and assigned sample of diverse individuals - to help allay these limitations.

This study represents the first time the MITI was utilized to measure the quality with school-based personnel who implemented MI with a sample of teachers. Unfortunately, subsequent analyses of MITI ratings identified substandard indicators of interrater reliably. Specifically, interclass correlations (ICC; utilizing the entire data set from coach-parent, and coach-teacher recordings; $n=43$ ) were calculated for behavioral counts and summary scores, while item level Kappa statistics were calculated for each rating of the global domain items. These statistics revealed that only two of six ICC statistics for the behavior count categories of Closed and Open Questions, rose above .85; while only one of four ICC statistics for behavior count summary scores of the Reflections to Questions ratio, was acceptable at .86. The global domain item Kappa statistics fared much worse, with no Kappa statistic rising above .06. Low interrater reliability may be a function of the instrument itself, as the MITI is more typically applied in direct service models (clinical counseling for aberrant behavior) or the result of poor inter-rater calibration efforts. Currently, steps are being taken to address these issues, prior to the re-coding of all audio-recorded sessions. First, our research team addressed 
the changes to the MITI and established new definitions that we believe clarify the global domains and behavior count categories more definitively (See Appendix O). Second, the following coding practices have been requested of the Clinical Training Institute; the establishment of reliability on practice recordings prior to coding actual recordings; frequent checks for "coder drift," which can be defined as any change that takes place between trained coders during the coding process; and reliability checks after every 10 recordings have been coded. This newly defined process is currently under way (April, 2012), the results of which are not yet available for this dissertation.

An additional limitation to a full understanding of the interventions impact on children rises from the absence of achievement data. As the goal for any school-based intervention is the overall benefit to children's ability to learn and thus achieve, the lack of measurement of this key outcome provide only an incomplete picture of the full impact of these enhancements.

\section{Implications for Practice}

The Motivational Interviewing Navigation Guide (MING). The conceptual model used to guide intervention procedures was developed in conjunction with the enhancements to the First Step homeBase intervention, and have been formalized and submitted for publication (Frey et al., nd.). The MING provides a conceptual map for intervention development and can be used to create the procedures to be followed in the application of MI, or as a tool for practitioners, supervisors, teachers, and students to develop and hone their existing MI skills. Based on Miller and Moyers (2006) eightstages of learning motivational interviewing, the MING includes five steps; and includes numerous interviewing techniques that are particularly useful to apply motivational 
interviewing in a directive fashion. These steps include: 1) complete values discovery and current practices interview, 2) collect fidelity data, 3) provide performance feedback, 4) implement extended consultation, education and support (optional), and 5) Closure.

First Step to Success Enhancements Manual. The conceptualization and development of the MING has allowed for the articulation all of our intervention procedures with far greater precision than was previously the case, and allowed the procedures and tools across the home and school components of the intervention to be structurally identical. A fourth iteration of the intervention manual has been written, and its Table of Contents can be found in Appendix P. The fourth iteration of the manual, includes a number of changes to the Enhanced homeBase and First Step CCU protocols. Many of these changes were the result of reorganizing the MING, and using the 5 universal principles as our "target behaviors" to be address parent and teacher behavior across the home and school applications. Our research team is currently utilizing the results from the final year of the Enhanced First Step to Success grant to inform changes to various components of the intervention, and to develop the $5^{\text {th }}$ iteration of the manual itself. Changes from the $4^{\text {th }}$ to the $5^{\text {th }}$ iteration of the manual include revisions to; (a) the Evoking Change Talk primers, which include foundational knowledge necessary for those implementing the program; (b) various tools, including Importance and Confidence Rulers, parent and teacher Values Discovery Activity Cards, and the graphics used during FSCCU and homeBase performance feedback routines; (c) and updates to the Parent and Teacher Current Practices Interviews. 


\section{Implications for Research}

Provided in this section are suggestions for future research in this area including suggestions for the revision of the measurement protocols used for this study, ideas for future replication, possible research questions, and additional lines of research that are possible given the findings described herein.

Revised Measurement Protocols. Although multiple measurement protocols were utilized in the completion of this research, the following are particularly important to future research application. First, The enhancements to the First Step to Success program have increased the complexity of the coach-teacher relationship, and may be reason for the modification of the ten-item version of the Coach-Teacher Alliance Survey (Walker et al., 2009; Sumi et al., in press). In particular, the coach-teacher relationship could be measured at baseline and post intervention, while added survey items to investigate the coaches' and teachers' perceptions of MI skill and strategy use, and its influence on perceived strength of the relationship would be informative. These insights would be particularly relevant to coaches, whose initial work with teachers is to build a working alliance. Currently, this survey does not ask respondents to relate the nuances of MI practice to their perceptions of the coach-teacher relationship. For example, specific questions relating to the Values Discovery Exercise, and its relationship to the teacher's and coach's perception of its benefits to their relationship might help address the critique of this exercise that was provided by teachers during focus group interviews. Questions such as these may best be addressed in an open response format (e.g., focus group interviews) allowing respondents to provide unrestricted answers. 
Second, changes to the Observation of Teacher Behavior System, which presents data categorically (focus student, peer, and class as a whole) could facilitate an exploration of the true nature of change in teacher behavior prior to and after the application of the intervention. During the Focus Group Interviews, teachers provided suggestions for improvements to this system, which could benefit added analyses, and support more detailed findings. One suggestion included a definitional change to the category of "peer," while another represented the importance of the "non-verbal" behavior of teachers within the classroom. First, during the Observation of Teacher Behavior behavioral counts in the category of peer are tallied for praise or reprimand statements directed at "any other peer in the class." As currently defined, "peer" includes numerous children other than the focus child and does not allow for a logical comparison of behavior counts between the focus child category, and $a$ "peer." Changing this category definition to "a peer without behavior problems" will allow for direct comparison of behavior tallies between the two implied categories- a child with serious school adjustment and behavior problems and a child without. Second, many teachers from our sample subscribe to the premise that all praise and reprimands are not all verbal in nature. Teachers have argued the current definition, which includes "verbal statements," does not provide a realistic count of the praise and reprimands experienced by children in their classrooms. The inclusion of "non-verbal teacher behavior" is supported in the current literature on this subject (Scott, Alter, \& Hirn, 2011), and may provide a more realistic picture of teacher behavior. These suggestions support the purpose of the Observation of Teacher Behavior system and are logical next steps in the development of a system designed to provide teachers with the data needed to motivate behavior change. 
Third, the use of the revised MITI (Moyers et al., 2007) may also require attention in future research. Professional coders, trained and experienced in the use of the MITI, were contracted to code all audio recordings of coach-teacher interactions. The positive results from this work are challenged by low inter-rater reliability. The MITI was modified for use in the broader Enhanced First Step research project. Three existing global ratings were modified to insure mutual exclusivity, removing multi-dimensional aspects, and stem and response options were modified to represent the ideal (i.e., high fidelity on the MITI) with items anchored on a Likert-type scale (strongly disagree strongly agree). The MITI author, Dr. Terri Moyers, assisted with the languaging of these to better reflect our indirect service delivery model and psycho-educational application. Our current calibration efforts represent the first attempts to explore the MITI's functionality within an indirect, psycho-educational service delivery option, and are a central focus for the continuation of this research line. These efforts are described in detail in the upcoming article; Transporting Motivational Interviewing to School Settings (Frey et al., nd), which is to be published in a special edition of the Journal of Applied School Psychology.

Fourth, a brief description of our work to modify the Teacher Motivation Inventory, (Nock \& Ferriter, 2005; Nock \& Kazdin, 2005; Nock \& Photos, 2006). This tool is not reported in within the current study, as it was initially deployed and then removed after we identified problems with the measure, as neither teacher nor parent motivation to change, based on Parent Motivation Inventory items (Nock \& Kazdin, 2005), changed in the desired direction. After a careful review we discovered, these items addressed motivation for the child to change (e.g., "My child's behavior has to improve 
soon") rather than motivation to participate in the intervention process and change parenting/teaching behavior, which is the focus of the intervention. The measure was revised using a motivational interviewing lens focused on adult behavior change. Additionally, we were initially collecting these at baseline and posttest. However, the change in motivation, according to our logic model, should take place a few weeks into the intervention. Thus, we decided to collect this measure alone after the feedback interview with parents and teachers.

Finally, the importance of measurement of academic achievement cannot be overlooked. The purpose of any school-based intervention is to affect change in the child's learning and thus achievement. The current study did not measure this final outcome, only a proxy measure of academic engaged time. Although academic engagement has demonstrated strong relationships with children's eventual achievement, future study (particularly in terms of the interventions efficacy) should include direct measures of achievement. As the intervention is relatively brief (on average three months) progress monitoring tools may be significantly more sensitive to children's changing achievement levels than more standardized measures commonly utilized.

Replication. Not only does the larger Enhanced First Step study represent the first rigorous evaluation of MI as applied in educational contexts, but also the first efforts to train school personnel to use MI. Researchers interested in replicating this study or elements of this study are cautioned to attend to the prerequisite skills of interventionists, and to provide training and coaching when necessary. The training and coaching of First Step behavior coaches was rigorous for this study, and lasted over the course of one school year. This level of intensity was necessary in order for our coaches to reach 
proficiency levels comparable to counselors using MI in more traditional environs. The amount of coaching and support required to teach someone who does not possess requisite MI skills in the following areas is likely to challenge training resources available within most school systems: (1) the desire to develop and maintain a therapeutic working alliance and sustain the requisite spirit of MI (as generally described within client centered therapy); (2) the capacity to engage teachers in productive problem solving and implications of the public education system; or (3) a basic understanding of behavioral assessment and contingency management (typical of operant and classical learning theory).

Future Research Questions. Future use of questionnaires to examine social validity issues may benefit from more specific questions related directly to the individual steps of the intervention process. Future studies should include in-depth focus group interviews with coaches in order to identify any suggested improvement to intervention procedures. It may also be that the in-depth information needed to understand this issue must to be acquired both qualitatively and quantitatively, and from coaches who have not been instrumental in the development of the intervention. Results could look very different with coaches who are exposed to the final FSCCU manual initially.

The Observation of Teacher Behavior has been modified from the original work of Reinke et al., (2008) to measure key teacher behaviors across the focus child, peers, and the class as a whole. Future use of this tool could concentrate on answering the following questions within the First Step intervention process:

- Was the teacher's shifting frequency of negative interactions to more positive interactions evenly distributed across these three agents? 
- Were the increases that were reported in aggregate due to increases in positive interactions with one or another of these three children?

- What effect did these shifts in interaction patterns (if any) have for the relationship between the teacher and the focus child?

Answering these questions is the next logical step in the application of the FSCCU with teachers, and for the clarification and modification of the logic model as presented here.

Finally, this study provided the first exploration of teacher-child relationships from within an enhanced version of the First Step to Success program that is designed to effect change in children with serious problem behavior. The results provide some indication that teachers' perceptions of the relationship with the focus child can change over the course of the intervention. At the very least, these findings indicate that systematic attention to the teacher-child relationship should be a necessary component of future research.

Additional Lines of Research. The focus on applying MI strategies with teachers has been fruitful, particularly with teachers of children who have serious problem behavior, and may be a unique opportunity for an entire line of research related to its use in school contexts. Adherence and quality results are important as they suggest this is a feasible approach within the schools, that our training and supervision of typically educated school personnel (e.g., a master's level teacher, counselor, and social worker) were successful, and open the possibility that AMIs might be effective across a variety applications in schools and educational consultations. The observation protocols and teacher feedback routines that have been developed during this work are unique and promising, and have a wide applicability for special education classrooms, itinerant 
services and in IEP staffings. Moreover, this work has been successful with the families of these children, and may be equally beneficial in enhancing the motivation of parents to engage in their children's education across a variety school related areas. This work is currently the focus of numerous grant applications currently under review by Institute of Educational Sciences (IES), while additional grant applications are being developed that focus primarily on training educational personnel to use MI in a variety of contexts.

\section{Conclusion}

The First Step to Success early intervention program (Walker, 1998) is a secondary prevention intervention that targets primary grade children with moderate or emerging behavior disorders. While the effectiveness of the First Step to Success early intervention program has been documented repeatedly (see Loman, Rodriguez, \& Horner, 2010; Walker et al., 2009), it has also been shown to be less effective with more severely disordered children and has a less dramatic impact on behavior in the home than in the school setting. In addition, many of the positive gains children demonstrate immediately following implementation of the First Step to Success program tend to fade once the intervention is discontinued if monitoring and booster sessions are not provided over the long term (See final IES report for: Evidence-based Interventions for Severe Behavior Problems--First Step to Success, March 2010). The current study, focused on motivational techniques to enhance teachers' use of feedback to interrupt the cycle of coercive interactions typically seen with children who demonstrate serious school adjustment, behavioral, and learning challenges, supports three important efforts. It was believed that teacher behavior change will more likely be sustained into the later phases of the First Step CLASS component of the intervention, and therefore increase the 
potential for sustaining the effects of the program after the fading of external reinforcers. This dissertation examined enhancements to the First Step to Success early intervention program, which rely heavily on the infusion of MI (Miller \& Rollnick, 2002) practices, focusing on the classroom teacher's use of praise to help replace the systematic use of external reinforcers; and to reduce the negative attention for inappropriate behavior (reprimands), which inadvertently maintains the challenging behavior teachers seek to eliminate. The resulting enhancement, the First Step Classroom Check-up, was largely based on the original work of Reinke et al., (2008). An open multiple-case-study design (Meyers et al., 2007) was used to investigate the intervention's social feasibility and acceptability, implementation fidelity.

As has been the case for over two decades, the First Step to Success program - in this case including enhancements to the original CLASS and homeBase components of the program -resulted in significant improvements in children's problem behaviors, social skills and observed academic engaged time. The continued success of this program, including high levels of social acceptance by the agents involved in its delivery, provides a foundation upon which a school wide application of the program's foundational tenants could be developed and tested.

The observed increase in teachers' use of praise and decreased reprimands, along with overall positive responses in terms of the interventions social validity, and positive child outcomes provide support for the integration of the Classroom Check-up (Reinke et al., 2008) into an Enhanced version of the First Step to Success Early Intervention Program. These outcomes also demonstrate the promise of future investigations of these 
interventions separately, and as combined and the probability that the efficacy of the intervention could be investigated.

During the intervention, teachers' perception of their relationship with the focus child changed for the better in terms of reduced Conflict, but was not as malleable for increases in Closeness. As an initial investigation of this relationship within the Enhanced intervention, these result call into question this relationship's potential to moderate or mediate the effectiveness of the interventions CLASS component, and seems worthy of further investigation. 


\section{REFERENCES}

Achenbach, T. (1991). The Child Behavior Checklist: Manual for the teacher's report form. Burlington: University of Vermont, Department of Psychiatry.

Adelman, H., \& Taylor, L. (2007). Systemic Change for School Improvement. Journal of Educational \& Psychological Consultation, 17(1), 55-77.

Aubrey, L. (1998). Motivational interviewing with adolescents presenting for outpatient substance abuse treatment (Unpublished doctoral dissertation). University of New Mexico. Albuquerque, NM.

Baker, J. (2006). Contributions of teacher-child relationships to positive school adjustment during elementary school. Journal of School Psychology, 44(3), 211229.

Beard, K. (1998). The effects of a teacher and parent directed proactive early intervention on the behaviors of elementary school children at risk for antisocial behavior (Doctoral dissertation, University of Oregon, 1998). Retrieved from Dissertation Abstracts International. $(59,157)$

Beauchaine, T., Webster-Stratton, C., \& Reid, M., J. (2005). Mediators, moderators, and predictors of 1-year outcomes among children treated for early-onset conduct problems: A latent growth curve analysis. Journal of Consulting and Clinical Psychology, 73(3), 371-388.

Benner, G., Nelson, J., Allor, J., Mooney, P., \& Dai, T. (2008). Academic processing speed mediates the influence of both externalizing behavior and language skills 
on the academic skills of students with emotional disturbance. Journal of Behavioral Education, 17(1), 63-78.

Bernal, M. (1984). Consumer issues in parent training. In R. Dangel and R. Polster (Eds.), Parent Training: Foundations of research and practice (pp. 447-501). NY: Guilford Press.

Bien, T., Miller, W., \& Boroughs, J. (1993). Motivational interviewing with alcohol outpatients. Behavioral and Cognitive Psychotherapy, 21, 347-356.

Birch, S., \& Ladd, G. (1996). Interpersonal relationships in the school environment and children's early school adjustment: The role of teachers and peers. In K. R. Wentzel \& J. Juvonen (Eds.), Social motivation: Understanding children's school adjustment (pp. 199-225). New York: Cambridge University Press.

Birch, S., \& Ladd, G. (1997). The teacher-child relationship and children's early school adjustment. Journal of School Psychology, 35(1), 61-79.

Braun, V, \& Clarke, V. (2006). Using thematic analysis in psychology. Qualitative Research in Psychology, 3, 77-101.

Bronfenbrenner, U. (1979). The ecology of human development: Experiments by nature and design. Cambridge, MA: Harvard University Press.

Bronfenbrenner, U. (1986). Ecology of the family as a context for human development: Research perspectives. Developmental Psychology, 22(6), 723-742.

Bronfenbrenner, U. (1989). Ecological systems theory. In R. Vasta (Ed.), Six theories of child development: Revised formulations and current issues (pp. 185-246).

Greenwich, CT: JAI Press. (Republished in 1992 under the same title, pp. 187250. London and Philadelphia: Jessica Kingsley Publishers). 
Bronfenbrenner, U., \& Ceci, S. (1994). Nature-nurture reconceptualized in developmental perspective: A bioecological model. Psychological Review, 101, $568-586$.

Bronfenbrenner, U. \& Morris, P. (2006). The bioecological model of human development. In R. M. Lerner (Ed.), Theoretical models of human development. Handbook of child psychology (Vol. 1, 6th ed.). Editors-in-chief: W. Damon \& R. M. Lerner. Hoboken, NJ: Wiley.

Brown, J., \& Miller, W. (1993). Impact of motivational interviewing on participation and outcome in residential alcoholism treatment. Psychology of Addictive Behaviors, 7,211-218.

Burchinal, M., Peisner-Feinberg, E., Pianta, R., \& Howes, C. (2002). Development of academic skills from preschool through second grade: Family and classroom predictors of developmental trajectories. Journal of School Psychology, 40, 415436.

Burke, B., Arkowitz, H. \& Menchola, M. (2003). The efficacy of motivational interviewing: A meta-analysis of controlled clinical trials. Journal of Consulting and Clinical Psychology, 71(5), 843-61.

Burns, B., \& Hoagwood, K. (2002). Community treatment for youth: Evidence-based interventions for severe emotional and behavioral disorders. New York: Oxford University Press.

Buyse, E., Verschueren, K., Doumen, S., Van Damme, J., \& Maes F. (2008). Classroom problem behavior and teacher-child relationships in kindergarten: The moderating role of classroom climate. Journal of School Psychology, 46(4), 367-391. 
Carr, J. E., Austin, J. L., Britton, L. N., Kellum, K., \& Bailey, J. S. (1999). An assessment of social validity trends in applied behavior analysis. Behavioral Interventions, $14(4), 223-231$.

Carter, D., \& Horner, R. (2007). Adding functional assessment to First Step to Success: A case study. Journal of Positive Behavioral Interventions, 9, 229-238.

Carter, D., \& Horner, R. (2009). Adding function based behavioral support to First Step to Success: Integrating individualized and manualized practices. Journal of Positive Behavioral Interventions, 11, 22-34.

Conroy, M. A., Dunlap, G., Clarke, S., \& Alter, P. J. (2005). A descriptive analysis of positive behavioral intervention research with young children with challenging behavior. Topics in Early Childhood Special Education, 25, 157-166.

DeRosier, M., Cillessen, A., Coie, J., \& Doge, K. (1994). Group social context and children's aggressive behavior. Child Development, 65, 1068-1079.

Diken, I., \& Rutherford, R. (2005). First Step to Success early intervention program: A study of effectiveness with Native-American children. Education \& Treatment of Children, 28(4), 444-465.

Dishion, T., McCord, J., \& Poulin, F. (1999). When interventions harm: Peer groups and problem behavior. American Psychologist, 54(9), 755-764.

Dishion, T., \& Stormshak, E. (Eds.). (2007). Intervening in children's lives: An ecological, family-centered approach to mental health care. Washington, DC: American Psychological Association.

Dishion, T., Stormshak, E., \& Siler, C. (2010). An ecological approach to interventions with high-risk students in schools: Using the Family Check-up to motivate parents' 
positive behavior support. In M. R. Shinn \& H. M. Walker (Eds.), Interventions for achievement and behavior problems in a three-tier model including RTI (pp. 101-124). Bethesda, MD: National Association of School Psychologists.

Dunn, C., DeRoo, L., \& Rivara, F. (2001). The use of brief interventions adapted from motivational interviewing across behavioral domains: A systematic review. Addiction, 96, 1725-1742.

Elliott, S., Gresham, F., \& Frank, J. (2008). Intervention validity of social behavior rating scales: Features of assessments that link results to treatment plans. Assessment for Effective Intervention, 34(1), p. 15-24.

Farran, D. (2000). Another decade of intervention for children who are low income or disabled: What do we know now? In J. P. Shonkoff, \& S. J. Meisels, (Eds), Handbook of early childhood intervention (2nd ed., pp. 510-548). New York, NY: Cambridge University Press.

Fixsen, D., Naoom, S., Blase, K., Friedman, R., \& Wallace, F. (2005). Implementation research: A synthesis of the literature (FMHI Publication \#231). Tampa, FL: University of South Florida, Louis de la Parte Florida Mental Health Institute, The National Implementation Research Network.

Forgatch, M., \& Patterson, G. (1998). Behavioral family therapy. In F. M. Dattilio (Ed.), Case studies in couple and family therapy: Systematic and cognitive perspectives (pp. 85-107). New York: Guilford Press.

Fraser, M. (2004). Risk and resilience in childhood an ecological perspective (2nd ed.). National Association of Social Workers: Washington, DC. 
Frederickson, B., \& Losada, M. (2005). Positive affect and the complex dynamics of human flourishing. American Psychologist, 60, 678-686.

Frey, A., Cloud, R., Lee, J., Small, J., Seeley, J., Fiel, E., Walker, H., \& Golly, A. (2011). The promise of motivational interviewing in school mental health. School Mental Health, 3(1), 1-12.

Frey, A. J., Park, K., Browne-Ferrigno, T., \& Korfhage, T. L. (2010). The Social Validity of Program-Wide Positive Behavior Support. Journal Of Positive Behavior Interventions, 12(4), 222-235.

Frey, A., Lee, J., Walker, H., Seeley, J., Small, J., Feil, E. (in press). The Motivational Interviewing Navigation Guide: A Process for Enhancing Teachers' Motivation to Adopt and Implement School-based Interventions. Journal of Educational and Psychological Consultation. Manuscript accepted for publication.

Frey, A., Seeley, J., Small, J., Feil, E., \& Walker, H. (in press). The feasibility and social validity of first step to success with preschoolers. Journal of Early Intervention. Manuscript accepted for publication.

Garbarino, J. (1992). Children and families in the social environment (2nd ed.). New York: Aldine de Gruyter.

Garbarino, J. (2001) Lost boys: Why our sons turn violent and how we can save them. Smith College Studies in Social Work Volume 71(2), 167-181.

Gardner, F., Connell, A., Trentacosta, C., Shaw, D., Dishion, T., \& Wilson, M. (2009). Moderators of outcome in a brief family centered intervention for preventing early problem behavior. Journal of Consulting and Clinical Psychology, 77, 543553. 
Gawande, A. (2009). The checklist manifesto: How to get things right. New York, NY: Metropolitan Books.

Golly, A. (2006). Five universal principles of positive behavior support. Verona, Wisconsin: Attainment Company, Inc.

Golly, A., Sprague, J., Walker, H., Beard, K., \& Gorham, G. (2000). The First Step to Success program: An Analysis of outcomes with identical twins across multiple baselines. Behavioral Disorders, 25, 170-182.

Golly, A., Stiller, B., \& Walker, H. (1998). First Step to Success: Replication and social validation of and early intervention program for achieving secondary prevention goals. Journal of Emotional and Behavioral Disorders, 6(4), 243-250.

Greenberg, M., Domitrovich, C., \& Bumbarger, B. (1999). Preventing mental disorders in school-age children: A review of the effectiveness of prevention programs. Available from the prevention Research Center for the Promotion of Human Development, Pennsylvania State University, State College, PA 16802.

Gresham, F.M. (1989). Assessment of treatment integrity in school consultation and prereferral intervention. School Psychology Review, 18, 37-50.

Greenwood, C., Delquadri, J. (1995). Class wide peer tutoring and the prevention of school failure. Preventing School Failure, 39(4) 21-26.

Gresham, F., \& Elliott, S. (1990). The social skills rating system (SSRS). Circle Pines, MN: American Guidance Service.

Gresham, F., \& Elliott, S. (2008). Social skills improvement system. Circle Pines, MN: Pearson Assessment. 
Gresham, F., Elliott, S., Cook, C., Vance, M., \& Kettler, R. (2010). Cross-informant agreement for ratings for social skill and problem behavior ratings: An investigation of the Social Skills Improvement System-rating scales. Psychological Assessment, 22(1), 157-166.

Gresham, F., Elliott, S., \& Kettler, R. (2010). Base rates of social skills acquisition/performance deficits, strengths, and problem behaviors: An analysis of the Social Skills Improvement System-rating scales. Psychological Assessment, 22(4), 809-815.

Gresham, F., Vance, M., Elliott, S., \& Cook, C. (2011). Comparability of the Social Skills Rating System to the Social Skills Improvement System: Content and psychometric comparisons across elementary and secondary age levels. School Psychology Quarterly, 26(1), 27-44.

Gunter, P., \& Coutinho, M. (1997). Negative reinforcement in classrooms: What we're beginning to learn. Teacher Education and Special Education, 20, 249-264.

Gunter, P., Denny, R., Jack, S., Shores, R., \& Nelson, C. (1993). Aversive stimuli in academic interactions between students with serious emotional disturbance and their teachers. Behavioral Disorders, 18, 265-274.

Gunter, P., Denny, R., Shores, R., Reed, T., Jack, S., \& Nelson, C. (1994). Teacher escape, avoidance, and countercontrol behaviors: Potential responses to disruptive and aggressive behaviors of students with severe behavior disorders. Journal of Child and Family Studies, 3, 211-223. 
Hamre, B., \& Pianta, R. (2001). Early teacher-child relationships and the trajectory of children's school outcomes through eighth grade. Child Development, 72, 625638.

Hettema, J., Steele, J., \& Miller, W. (2005). Motivational Interviewing. Annual Review of Clinical Psychology, 1, 91-111.

Hieneman, M., \& Dunlap, G. (2000). Factors affecting the outcomes of community-based behavioral support: II. Factor category importance. Journal of Positive Behavior Interventions, 3, 67-74.

Hieneman, M., Dunlap, G., \& Kincaid, D. (2005). Positive support strategies for students with behavioral disorders in general education settings. Psychology in the Schools, $42(8), 2005$.

Hoagwood, K. (2001). Evidence-based practice in children's mental health services: What do we know? Why aren't we putting it to use? Report on Emotional \& Behavioral Disorders in Youth, 1(4), 84-88.

Hoagwood, K., Olin, S., Kerker, B., Kratochwill, T., Crowe, M., \& Saka, N. (2007). Empirically based school interventions targeted at academic and mental health functioning. Journal of Emotional and Behavioral Disorders, 15(2), 66-92.

Hops, H. (1978). CLASS: A standardized in-class program for acting-out children: II. Field test evaluations. Journal of Educational Psychology, 70(4), 636-644.

Hops, H., \& Walker H. (1988). CLASS: Contingencies for Learning Academic and Social Skills. Seattle, WA: Educational Achievement. Systems. 
Hughes, J., Cavell, T., \& Jackson, T. (1999). Influence of teacher-student relationships on aggressive children's development: A prospective study. Journal of Clinical Child Psychology 28, 173-184.

Hughes, J., Gleason, K., \& Zhang, D. (2005). Relationship influences on teachers' perceptions of academic competence in academically at-risk minority and majority first grade students. Journal of School Psychology, 43(4), 303-320.

Jacobson, N., \& Truax, P. (1991). Clinical significance: A statistical approach to defining meaningful change in psychotherapy research. Journal of Consulting \& Clinical Psychology, 59(1), 12-19.

Jennings, P., \& Greenberg, M. (2009). The prosocial classroom: Teacher social and emotional competence in relation to student and classroom outcomes. Review of Educational Research, 79(1), 491-525.

Jenson, J., \& Fraser, M. (2006) Child mental health policy. In J. Jenson \& M. Fraser (Eds.), Social Policy for Children and Families (pp. 103-130). Thousand Oaks, CA: Sage Publication.

Jenson, W., Olympia, D., Farley, M., \& Clark, E. (2004). Positive psychology and externalizing students in a sea of negativity. Psychology in the Schools, 41(1), 6779.

Jerome, E., Hamre, B., \& Pianta, R. (2009). Teacher-child relationships from kindergarten to sixth grade: Early childhood predictors of teacher-perceived conflict and closeness. Social Development, 18(4), 915-945. 
Jones, K., Wickstrom, K., \& Friman, P. (1997). The effects of observational feedback on treatment integrity in school-based behavioral consultation. School Psychology Quarterly, 12, 316-326.

Kazdin, A. (1987). Conduct disorders in childhood and adolescence. London: Sage. Koomen, H., Verschueren, K., \& Pianta, R. (2007). Leerling-Leerkracht Relatie Vragenlijst (LLRV): Handleiding. [Student-Teacher Relationship Scale: Manual.] Houten, The Netherlands: Bohn Stafleu van Loghum.

Kutash, K., Duchnowski, A., \& Lynn, N. (2006). School-based mental health: An empirical guide for decision-makers. Tampa, FL: University of South Florida, The Louis de la Parte Florida Mental Health Institute, Department of Child and Family Studies, Research and Training Center for Mental Health.

Ladd, G., Birch, S., \& Buhs, E. (1999). Children's social and scholastic lives in Kindergarten: Related spheres of influence? Child Development, 70(6), 13731400.

Ladd, G., \& Burgess, K. (1999). Charting the relationship trajectories of aggressive, withdrawn, and aggressive/withdrawn children during early grade school. Child Development, 70(4), 910-929.

Lane, K., Gresham, F., O'Shaughnessy, T. (2002) Interventions for children with or atrisk for emotional and behavioral disorders. Boston: Allyn \& Bacon.

Lane, K.L., \& Beebe-Frankenberger, M. E. (2004). School-based interventions: The tools you need to succeed. Boston, MA: Allyn \& Bacon 
Leff, S., Power, T., Manz, P., Costigan, T., \& Narbors, L. (2001). School-based aggression prevention programs for young children: Current status and implications for violence prevention. School Psychology Review, 30(3), 344-362.

Lien-Thorne, S., \& Kamps, D. (2005). Replication study of the First Step to Success early intervention program. Behavioral Disorders, 31, 18-32.

Loman, S., Rodriguez, B., \& Horner, R. (2010). Sustainability of a targeted intervention package: First Step to Success in Oregon. Journal of Emotional \& Behavioral Disorders, 18(3), 178-191.

Lundahl, B., Tollefson, D., Kunz, C., Brownell, C., \& Burke, B. (2010). Meta-analysis of motivational interviewing: Twenty-five years of research. Research on Social Work Practice, 20, 137-160.

Lunkenheimer, E.; Shaw, D., Gardner, F., Dishion, T., Connell, A., \& Wilson, M. (2008). Collateral benefits of the Family Check-up on early childhood school readiness: Indirect effects of parents' positive behavior support. Developmental Psychology, $44,1737-1752$.

Maag, J., (2001). Rewarded by punishment: Reflections on the disuse of positive reinforcement in schools. Exceptional Children 67(2), 173-186.

Madson, M., \& Campbell, T. (2006). Measures of fidelity in motivational enhancement: A systematic review. Journal of Substance Abuse Treatment, 31(1), 67-73.

Masten, A. (1987). Resilience in development: Implications of the study of successful adaptation for developmental psychopathology. In C. Cicchetti (Ed), The emergence of a discipline: Rochester symposium on developmental psychopathology (pp. 261-294). Hillsdale, NJ: Lawrence Erlbaum. 
Masten, A., \& Gewirtz, A. (2006). Vulnerability and resilience in early child development. In K. McCartney \& D. A. Phillips (Eds.). Handbook of early childhood development (pp. 22-43). Malden, Massachusetts: Blackwell.

McEvoy, A., \& Welker, R. (2000). Antisocial behavior, academic failure, and school climate: A critical review. Journal of Emotional and Behavioral Disorders, 8(3), $130-140$.

Meehan, B., Hughes, J., \& Cavell, T. (2003). Teacher-student relationships as compensatory resources for aggressive children. Child Development, 74, 11451157.

Miller, W. R., (2012). Equipoise and Equanimity in Motivational Interviewing. Motivational Interviewing: Training, Research, Implementation, Practice. 1 (1), p. 31-32. www.mitrip.org ISSN 2160-584X (online).

Meyers, J., Truscott, S. D., Meyers, A. B., Varjas, K., \& Collins, A. S. (2007). Qualitative and mixed methods designs in consultation research. In W. P. Erchul \& S. M. Sheridan (Eds.), Handbook of research in school consultation: Empirical foundations for the field (pp. 89-114). Hillsdale, NJ: Erlbaum.

Miller, W. (1985). Motivation for treatment: A review with special emphasis on alcoholism. Psychological Bulletin, 98, 84-107.

Miller, W., \& Rollnick, S. (2002). Motivational interviewing: Preparing people for change (2nd ed.). New York: Guilford Press.

Miller, W., \& Sovereign, R. G. (1989). The checkup: A model for early intervention in addictive behaviors. In T. Loberg, W. R. Miller, P. E. Nathan, \& G. A. Marlatt 
(Eds.), Addictive behaviors: Prevention and early intervention (pp. 219-231). Amsterdam: Swets \& Zeitlinger.

Moffitt, T. (1993). Adolescence-limited and life-course-persistent antisocial behavior: A developmental taxonomy. Psychological Review, 100(4), 674-701.

Moyers, T., Martin, T., Manuel, J., Hendrickson, S., \& Miller, W. (2005). Assessing competence in the use of motivational interviewing. Journal of Substance Abuse Treatment, 28, 19-26.

Moyers, T., Martin, T., Manuel, J., Miller, W., \& Ernst, D. (2007). The Motivational Interviewing Treatment Integrity (MITI) Code 3.0. Unpublished coding manual. University of New Mexico, Center on Alcoholism, Substance Abuse, \& Addictions.

Myers, S., \& Pianta, R. (2008). Developmental commentary: Individual and contextual influences on student-teacher relationships and children's early problem behaviors. Journal of Clinical Child \& Adolescent Psychology, 37(3), 600-608.

Nelson, J., Hurley, K., Synhorst, L., Epstein, M., Stage, S., \& Buckley, J. (2009). The child outcomes of a behavior model. Exceptional Children, 76, 7-30.

Nicholas, P., Olympia, D., \& Jenson, W. (2001). Saying and doing the right things: A comparison of teacher school psychologist intervention knowledge and competencies. Paper presented at the annual meeting of the National Association of School Psychologists, Washington, D.C.

Nock, M., \& Ferriter, C. (2005). Parent management of attendance and adherence in child and adolescent therapy: A conceptual and empirical review. Clinical Child and Family Psychology Review, 8, 149-166. 
Nock, M., \& Kazdin, A. (2005). Randomized controlled trial of a brief intervention for increasing participation in parent management training. Journal of Consulting and Clinical Psychology, 73, 872-879.

Nock, M., \& Photos, V. (2006). Parent motivation to participate in treatment: Assessment and prediction of subsequent participation. Journal of Child and Family Studies, $15,345-358$.

Noell, G., Witt, J., Slider, N., Connell, J., Gatti, L., Williams, K., ... Resetar, J. (2005). Treatment implementation following behavioral consultation in schools: A comparison of three follow-up strategies. School Psychology Review, 34, 87-106.

Noonan, W., \& Moyers, T. (1997). Motivational interviewing: A review. Journal of Substance Misuse, 2, 8-16.

Olson, S., Sameroff, A., Kerr, D., Lopez, N., \& Wellman, H. (2005). Developmental foundations of externalizing problems in young children: The role of effortful control. Development and Psychopathology, 17(1), 25-45.

Overton, S., McKenzie, L., King, K., \& Osborne, J. (2002). Replication of the First Step to Success model: A multiple case study of implementation effectiveness. Behavioral Disorders, 28, 40-56.

Papalia-Berardi, A., \& Hall, T. E. (2007). Teacher assistance team social validity: A perspective from general education teachers. Education and Treatment of Children, 30(2), 89-110.

Patterson, G. (1976). The aggressive child: victim and architect of a coercive system. In: E. J. Mash, L. A. Hamerlynck \& L. C. Handy (Eds.), Behavior modification and families. (pp. 267-316). New York: Brunner/Mazel. 
Patterson, G. (2002). The early development of coercive family process. In J. B. Reid, G. Patterson, \& J. Snyder (Eds.), Antisocial behavior in children and adolescents (pp. 25-44). Washington, DC: American Psychological Association.

Patterson, G., \& Reid, J. (1970). Reciprocity and coercion: Two facets of social systems. In C. Neuringer \&. J. L. Michael (Eds.), Behavior modification in clinical psychology (pp. 133-177). New York: Appleton.

Patterson, G., Reid, J., \& Dishion, T. (1992). Antisocial boys. Eugene, OR: Castalia Press. Patterson, G., \& Yoerger, K. (1997). A developmental model for late-onset delinquency. In D. W. Osgood (Ed.), Motivation and delinquency, (Vol. 44, pp. 121-177). Lincoln, NE: Nebraska Symposium on Motivation.

Peppler, D., Craig, W., \& Roberts, W. (1998). Observations of aggressive and nonaggressive children on the school playground. Merrill-Palmer Quarterly, 44, $55-76$.

Pianta, R. (2001). Student-teacher relationship scale. Professional manual. Lutz, FL: Psychological Assessment Resources.

Pianta, R., Nimetz, S., \& Bennett, E. (1997). Mother-child relationships, teacher-child relationships, and school outcomes in preschool and kindergarten. Early Childhood Research Quarterly, 12, 263-280.

Pianta, R., \& Stuhlman, M. (2004). Teacher-child relationships and children's success in the first years of school. School Psychology Review, 33(3), 444-458.

Pianta, R., Stuhlman, M., \& Hamre, B. (2002). How schools can do it better: Fostering stronger connections between teachers and students. New Directions for Youth Development, 93, 91-107. 
Quinn, M., Mathur, S., \& Rutherford, R. (1995). Early identification of antisocial boys: A multi-method approach. Education and Treatment of Children, 18, 272-281.

Reid, J. (1993). Prevention of conduct disorder before and after school entry: Relating interventions to development findings. Development and Psychopathology, 5, $243-262$.

Reid, J., \& Eddy, J. (1997). The prevention of antisocial behavior: Some considerations in search of effective interventions. In D. Stoff, J. Breiling, \& J. Maser (Eds.), The Handbook of Antisocial Behavior (pp. 343-356). New York: Wiley.

Reinke, W., \& Herman, K. (2002). Creating school environments that deter antisocial behaviors in youth. Psychology in the Schools, 39(5), p. 549-559.

Reinke, W., Lewis-Palmer, T., \& Martin, E. (2007). The effect of visual performance feedback on teacher use of behavior-specific praise. Behavior Modification, 31(3), 247-263.

Reinke, W., Lewis-Palmer, T., \& Merrell, K. (2008). The Classroom Check-up: A class wide teacher consultation model for increasing praise and decreasing disruptive behavior. School Psychology Review, 37(3), 315-332.

Renner, A. (2010). Classism and education: NCLB, regulated knowledge, and resistance. The Rouge Forum Newspaper (fall 2009 / winter 2010, issue 15). Retrieved online from http://www-rohan.sdsu.edu/ rgibson/rouge_forum/renner.htm, May 28, 2011.

Richman, J., \& Fraser, M. (2001). Resilience in childhood: The role of risk and protection. In J.M. Richman, \& M.W. Fraser (Eds.), The context of youth violence: Resilience, risk, and protection (pp. 1-12). New York: Prager Press. 
Rodriguez, B., Loman, S., \& Horner, R. (2009). A preliminary analysis of the effects of coaching feedback on teacher implementation fidelity of First Step to Success. Behavior Analysis in Practice, (Vol. 2, fall).

Rogers, C. R. (1959). A theory of therapy, personality, and interpersonal relationships as developed in the client-centered framework. In S. Koch (Ed.), Psychology: The study of a science: Formulations of the person and the social contexts (Vol. 3, pp. 184-256). New York: McGraw-Hill.

Rogoff, B. (2003). The cultural nature of human development. New York: Oxford University Press.

Rudasill, K. (2011). Child temperament, teacher-child interactions, and teacher-child relationships: A longitudinal investigation from first to third grade. Early Childhood Research Quarterly, 26(2), 147-156.

Rudasill, K., Reio, T., Stipanovic, N., \& Taylor, J. (2010). A longitudinal study of student-teacher relationship quality, difficult temperament, and risky behavior from childhood to early adolescence. Journal of School Psychology, 48(5), 389412

Rudasill, K., \& Rimm-Kaufman, S. (2009). Teacher-child relationship quality: The roles of child temperament and teacher-child interactions. Early Childhood Research Quarterly, 24, 107-120.

Russell Carter, D., \& Horner, R. (2007). Adding functional behavioral assessment to First Step to Success: A case study. Journal of Positive Behavior Interventions, 9, 229238. 
Sameroff, A., \& Fiese, B. (2000). Transactional regulation: The developmental ecology of early intervention. In J. Schonkoff \& S. Meisels (Eds.), Handbook of early childhood intervention (2nd ed., pp. 135-159). New York: Cambridge University Press.

Sameroff, A., \& Mackenzie, M. (2003). Research strategies for capturing transactional models of development: The limits of the possible. Development and Psychopathology, 15, 613-640.

Sanetti, L., \& Kratochwill, T. R. (2009). Toward Developing a Science of Treatment Integrity: Introduction to the Special Series. School Psychology Review, 38(4), 445-459.

Saunders, B., Wilkinson, C., \& Phillips, M. (1995). The impact of a brief motivational intervention with opiate users attending a methadone programme. Addiction, 90 , $415-424$.

Scott, T. M., Alter, P. J., \& Hirn, R. G. (2011). An Examination of Typical Classroom Context and Instruction for Students With and Without Behavioral Disorders. Education \& Treatment Of Children (West Virginia University Press), 34(4), 619641.

Shaw, D., Dishion, T., Supplee, L., Gardner, F., \& Arnds, K. (2006). Randomized trial of a family-centered approach to the prevention of early conduct problems: 2-Year effects of the Family Check-up in early childhood. Journal of Consulting and Clinical Psychology, 74, 1-9. 
Sheridan, S. M., Swanger-Gagné, M., Welch, G. W., Kwon, K., \& Garbacz, S. (2009). Fidelity Measurement in Consultation: Psychometric Issues and Preliminary Examination. School Psychology Review, 38(4), 476-495.

Shonkoff, J. P., \& Meisels, S. J. (Eds). Handbook of early childhood intervention (2nd ed.). New York, NY: Cambridge University Press.

Shonkoff, J. P., \& Phillips, D. (Eds.). (2000). From neurons to neighborhoods: The science of early childhood development. Washington, DC: The National Academy Press.

Shores, R., Gunter, P., \& Jack, S. (1993). Classroom management strategies: Are they setting events for coercion? Behavioral Disorders, 18, 92-102.

Silver, R., Measelle, J., Armstrong, J, \& Essex, M. (2005). Trajectories of classroom externalizing behavior: Contributions of child characteristics, family characteristics, and the teacher-child relationship during the school transition. Journal of School Psychology, 43, 39-60.

Silver, R., Measelle, J., Armstrong, J., \& Essex, M. (2010). The impact of parents, childcare providers, teachers, and peers on early externalizing trajectories. Journal of School Psychology, 48(6), 555-583.

Simpson G., Cohen R., Pastor P., Reuben C. (2008). Use of mental health services in the past 12 months by children aged 4-17 years: United States, 2005-2006. Hyattsville, MD: National Center for Health Statistics; NCHS data brief No. 8. Split, J., \& Koomen, H. (2009). Widening the view on teacher-child relationships: teachers' narratives concerning disruptive versus nondisruptive children. School Psychology Review, 38(1), 86-101. 
Sprague, J., \& Perkins, K. (2009). Direct and Collateral Effects of the First Step to Success Program. Journal of Positive Behavior Interventions, 11(4), 208-221.

Stagman, S., \& Cooper, J. (2010). Children's mental health: What every policymaker should know. Columbia University, NY: National Center for Children in Poverty.

Sumi, C., Woodbridge, M., Javitz, H., Thornton, P., Wagner, M., Rouspil, K. ... Severson, H. (In press). Assessing the effectiveness of First Step to Success: Are short-term results the first step to long-term behavioral improvements?

Suter, W. N. (2006). Introduction to educational research: A critical thinking approach. Thousand Oaks, CA: Sage.

Sutherland, K., \& Oswald, D. (2005). The relationship between teacher and student behavior in classrooms for students with emotional and behavioral disorders: Transactional processes. Journal of Child and Family Studies. p. 1-14.

Sutherland, K., Wehby, J., \& Copeland, S. (2000). Effect of varying rates of behaviorspecific praise on the on-task behavior of students with emotional behavioral disorder. Journal of Emotional \& Behavioral Disorders, 8(1), 2-8.

Schwartz, I. S., \& Baer, D. M. (1991). Social validity assessments: Is current practice state of the art? Journal of Applied Behavior Analysis, 24, 89-204.

Swick, K., \& Williams, R. (2006). An analysis of Bronfenbrenner's bioecological perspective for early childhood educators: Implications for working with families experiencing stress. Early Childhood Education Journal, 33(5), 371-378.

U.S. Department of Education, Institute of Education Sciences, What Works Clearinghouse. (2012, March). Children Classified as Having an Emotional 
Disturbance intervention report: First Step to Success. Retrieved from http://whatworks.ed.gov.

Vasilaki, E., Hosier, S., \& Cox, W. (2006). The efficacy of Motivational Interviewing as a brief intervention for excessive drinking: a meta-analytic review. Alcohol \& Alcoholism, 41, 328-335.

Wagner, C. C., (2012). Client-centered Direction Or How to Get There When You're Not Sure Where You're Going. Motivational Interviewing: Training, Research, Implementation, Practice. 1 (1), p. 31-32. www.mitrip.org ISSN 2160-584X (online).

Walker, H. (1998). First Step to Prevent Antisocial Behavior. Teaching Exceptional Children, 30, 16-19.

Walker, H., Colvin, G., \& Ramsey, E. (1995). Antisocial behavior in school: Strategies and best practices. Pacific Grove, CA: Brooks/Cole.

Walker, H., Golly, A., Kavanagh, K, Stiller, B., Severson, H., \& Feil, E. (2002). First Step to Success: Helping young children overcome antisocial behavior Preschool edition. Longmont, CO: Sopris West.

Walker, H., Golly, A., McLane, J., \& Kimmich, M. (2005). The Oregon First Step to Success replication initiative: State wide results of an evaluation of the program's impact. Journal of Emotional and Behavioral Disorders, 13(3), 163-172.

Walker, H., Horner, R., Sugai, G., Bullis, M., Sprague, J., Bricker, D., Kaufman, M. (1996). Integrated approaches to preventing antisocial behavior patterns among school-age children and youth. Journal of Emotional and Behavioral Disorders, 4(4), 194. 
Walker, H., Irvin, L., Noell, J., \& Singer, G. (1992). A construct score approach to the assessment of social competence: Rationale, technological considerations, and anticipated outcomes. Behavior Modification, 16, 448-474.

Walker, H., Kavanagh, K., Stiller, B., Golly, A., Severson, H., \& Feil, E. (1998). First Step to Success: An early intervention approach for preventing school antisocial behavior. Journal of Emotional \& Behavioral Disorders, 6(2), 66-81.

Walker, H., Ramsey, E., \& Gresham, F. (2004). Antisocial behavior in school: Evidencebased practice (2nd ed.). Belmont, CA: Wadsworth/Thompson Learning.

Walker, H., Seeley, J., Small, J., Severson, H., Graham, B., Feil, E., ... Golly, A. (2009). A randomized controlled trial of the First Step to Success early intervention: Demonstration of program efficacy outcomes in a diverse, urban school district. Journal of Emotional and Behavioral Disorders, 17(4): 197-212.

Walker, H., \& Severson, H. (1990). Systematic screening for behavior disorders: User's guide and technical manual. Longmont, $\mathrm{CO}$ : Sopris West.

Walker, H., Severson, H., \& Feil, E. (1994). The early screening project: A proven childfind process. Longmont, CO: Sopris West.

Walker, H., Severson, H., Nicholson, F., Kehle, T., Jenson, W., Clark, E. (1994). Replication of the systematic screening for behavior disorders procedure for the identification of at-risk children. Journal of Emotional and Behavioral Disorders, 2, 66-77.

Walker, H., Severson, H., Stiller. B., Williams, G., Haring, N., Shinn, M., Todis, B. (1988). Systematic screening of pupils in the elementary age range at risk for 
behavior disorders. Development and trial testing of a multiple gating model. Remedial and Special Education, 9(3), 8-20

Walker, H., Severson, H., Todis, B., Block-Pedego, A., Williams, G., Haring, N., Barckley, M. (1990). Systematic screening for behavior disorders: Further validation, replication, and normative data. Remedial and Special Education, 11, $32-46$.

Walker, H., Shinn, M., O'Neill, R., \& Ramsey, E. (1987). A longitudinal assessment of the development of antisocial behavior in boys: Rational, methodology, and first year results. Remedial and Special Education, 8(4), 7-16.

Walker, H. M., Sprague, J. R., Perkins-Rowe, K. A., Beard-Jordan, K. Y., Seibert, B. M., Golly, A. M., Severson, H. H., \& Feil, E. G. (2005). The First Step to Success program: Achieving secondary prevention outcomes for behaviorally at-risk children through early intervention. In M. H. Epstein, K. Kutash, \& A. J. Duchnowski (Eds.), Outcomes for children and youth with emotional and behavioral disorders and their families: Programs and evaluation best practices ( $2^{\text {nd }}$ ed., pp. 501-523). Austin, TX: PRO-ED.

Walker, H., Stiller, B., \& Golly, A. (1998). First Step to Success: A collaborative homeschool intervention for preventing antisocial behavior at the point of school entry. Young Exceptional Children, 1(2), 2-6.

Walker, H., Stiller, B., Golly, A., Kavanagh, K., Severson, H., \& Feil, E. (1997). First Step to Success: Helping children overcome antisocial behavior. Implementation guide. Longmont, CO: Sopris West. 
Woodcock, R., Mather, N., \& Schrank, F. (2004) Woodcock-Johnson III Diagnostic Reading Battery. Itasca, IL: Riverside Publishing.

Wyrick, A., \& Rudasill, K. (2009). Parent involvement as a predictor of teacher-child relationship quality in third grade. Early Education and Development, 20, 1-20.

Zigler, E., \& Taussig, C. (1992). Early childhood intervention. American Psychologist, 47, $997-1007$. 


\section{LIST OF APPENDICIES}

APPENDIX

PAGE

A. First Step to Success Program Description ..............................201

B. The Classroom Expectations Checklist...................................208

C. The First Step Classroom Check-up Action Plan...........................209

D. The First Step Classroom Check-up Process Fidelity Checklist..............210

E. Enhanced First Step Fidelity (MI Quality)...............................211

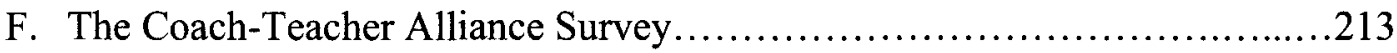

G. The Enhanced First Step CLASS Component Fidelity Checklist............215

H. The Enhanced First Step to Success CLASS Monitoring Log................217

I. The Student-Teacher Relationship Scale (short form) $\ldots \ldots \ldots \ldots \ldots \ldots \ldots \ldots . \ldots \ldots$

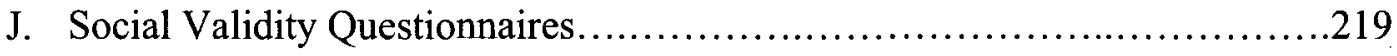

K. Focus Group Interview Questions...................................221

L. Case-level Outcome Results Tables.................................224

M. Recording Procedures for the Observation of Teacher Behavior.............228

N. MITI Global Rating Revision Description..............................233

O. The First Step to Success Enhancements Manual Table of Contents..........235

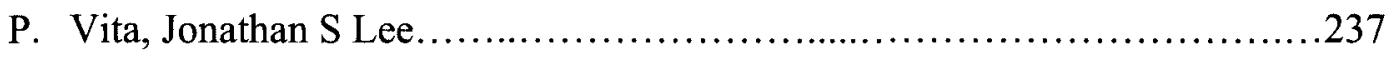




\section{Appendix A}

\section{First Step to Success Program Description ${ }^{1}$}

The First Step (FS) program was developed through a four-year, federal grant (1992-96) to Walker and associates from the Office of Special Education Programs of the U.S. Department of Education. Year one of the project was devoted to planning, design, trial testing, and refinement of the three FS component modules, as well as development of the necessary working relationships with the participating school district. Years two and three focused on implementing, evaluating, and refining the FS intervention. The final project year was devoted to long-term follow-up assessments, packaging, field-testing, dissemination, and staff training at the development site and beyond.

First Step consists of three modules designed to be applied in concert with each other. These are 1) universal screening (Walker, Severson, \& Feil, 1995); 2) the school module (Hops \& Walker, 1988); and 3) home module. The two primary goals of the FS intervention are to teach the at-risk child to get along with others (teachers and peers) and to engage assigned schoolwork in an appropriate, successful manner. The intervention is designed to achieve secondary prevention outcomes for children with moderate or emerging school adjustment and behavior challenges.

The three modules of FS are based on extensive research on school and home intervention procedures with aggressive, antisocial youth and over a decade of work

\footnotetext{
1 This description was taken and adapted, with permission, from the Enhanced First Step Project Narrative, and was originally authored by Dr. Hill Walker of the University of Oregon. 
related to the universal, proactive early screening of at-risk children to provide early detection (See McCord, 1993; Patterson et al., 1992; Hops \& Walker, 1988;

Walker, et al., 1988). Four options, varying in their complexity and required effort, are contained within the screening component. The most comprehensive of these options, the Systematic Screening for Behavior Disorders (Walker, et al., 1995), is proposed in this study and described in detail in Chapter 3 of this proposal. The school and home modules are described below.

\section{School Module (CLASS)}

The school intervention module of FS is an adapted version of the Contingencies for Learning Academic and Social Skills (CLASS) program developed by Hops and Walker, (1988) for use with conduct disordered students in the primary, elementary grades. CLASS requires 30 program days for successful completion. Each program day has a built in, performance criterion that has to be met before proceeding to the next day of the intervention program; if the criterion isn't met, that program day is then repeated and/or the student is recycled to an earlier, successfully-completed program day before proceeding on. Most students require approximately two months, minimum, to complete the CLASS program because of this built-in recycling procedure.

CLASS is divided into three successive phases: Behavioral Coach, Teacher, and Maintenance. The behavioral coach phase (program days 1-5) is the responsibility of an adult, trained as a FS behavioral coach, who coordinates the implementation process. This role is normally assumed by a related service provider (e.g., school counselor, school psychologist, school social worker or behavioral specialist), but can be the responsibility of a trained assistant, parent volunteer or graduate student. The role 
requires someone who can a) directly implement the program for brief portions of the school day and b) monitor, supervise and support participating teachers as they assume control of the program.

The CLASS program begins with two, twenty-minute periods daily, usually scheduled during a.m. and p.m. sessions, and are eventually extended to the entire school day. Initially, the behavioral coach, in close proximity to the focus child, monitors her or his classroom behavior using a red and green card on which one point is awarded every thirty seconds. If the child's behavior is appropriate when the point award interval occurs, the point goes on the green side of the card; if not, it goes on the red side. To meet the criterion, $80 \%$ or more of the available points during the twenty-minute period have to be awarded on the green side. A brief, free time activity involving the focus child and peers is made available immediately following the twenty-minute period. If the reward criterion for both a.m. and p.m. sessions is met, the child earns a home privilege as well that has been prearranged with parents or caregivers.

Over the course of the program, use of the red/green card is faded out completely by program day 15 and the interval in which points and praise can be earned is gradually extended from 30 seconds to ten minutes. In addition, in the later stages of the program, the focus child has to work in blocks of multiple days in order to earn a single reward of higher magnitude.

The Teacher Phase (Program Days 6-20) is operated by the classroom teacher in whose room the CLASS program is initially implemented. The regular, homeroom teacher assumes control of the program's operation on program day six but with close supervision and support provided by the behavioral coach. The behavioral coach provides 
monitoring and technical assistance as needed for the regular teacher throughout the remainder of the teacher phase. Teacher phase implementation tasks include: a) operating the program daily b) awarding praise and points according to program guidelines and contingent upon child performance c) supervising delivery of group activity, school rewards and d) communicating with parents on a regular basis regarding the focus child's performance. The regular teacher works closely with the program behavioral coach, child, parents and peers throughout the total implementation period.

The Maintenance Phase of the CLASS program lasts from Program Day 21 to 30 after which the school intervention is terminated. In this final program phase, the focus child is rewarded primarily with praise and expressions of approval/recognition from the teacher at school and the parents at home. An attempt is made during this phase to reduce the child's dependence upon the program by substituting adult praise for points, reducing the amount of daily feedback given and making occasional rewards available contingent upon exemplary performance.

The CLASS program was initially developed, tested and validated over a five year period and has been extensively researched (See Hops \& Walker, 1988; Walker, Hops, \& Greenwood, 1984); the program accomplishes powerful behavior change outcomes for acting out students at the point of school entry (Hops, et al., 1978).

\section{Home Module (homeBase)}

The homeBase component of FS consists of a series of six lessons designed to enable parents and caregivers to build child competencies and skills in six areas that affect school adjustment and performance. The target skills that parents are asked to teach their children are as follows: 1) Sharing School, 2) Cooperation, 3) Limit-Setting, 4) 
Problem-Solving, 5) Friendship-Making, and 6) Developing Confidence. HomeBase contains lessons, instructional guidelines, and parent-child games and activities for directly teaching these skills. HomeBase requires six weeks for implementation and begins after the focus child has completed program day ten of the CLASS program. The FS program behavioral coach visits the parents' home on a weekly basis and conducts the homeBase lessons in that setting. Following each session, materials are left with the parents that facilitate daily review and practice of each skill with the focus child. The homeBase lessons require approximately one hour each. Parents are encouraged to work with their child ten to fifteen minutes daily and to focus on practicing the homeBase skills being taught.

An important, shared goal of homeBase is to build a strong, positive link between home and school. HomeBase is designed to strengthen parenting skills in developing child competence in key performance areas related to school success. Parents and caregivers are enlisted as partners, with the school, in helping the child get off to the best possible start in his or her school career. Its ultimate goal is to unite educators and parents-caregivers in helping vulnerable children experience early school success.

It is important to note that parents are never blamed for the problems their child may be experiencing in school. Instead, developing a collaborative home and school working relationship whose focus is on joint problem solving and the development of school success is emphasized. This skill building approach is based on the belief that parents are children's best natural resource for achieving school success.

HomeBase content is based on over 25 years of research at the Oregon Social Learning Center (OSLC) involving hundreds of families who have contributed to our 
current knowledge of the family-based factors related to children's competent social adjustment (See Patterson, 1982; Patterson, Reid \& Dishion, 1992). The approach used in teaching parents how to improve their child's school success in homeBase reflects numerous OSLC clinical trials and research efforts to study the processes inherent in family based, behavior change processes (Dishion, Patterson, \& Kavanagh, 1992; Patterson, 1982). It also stresses the importance of developing a collaborative relationship with parents and "tailor-making" the delivery and implementation of the target skills to meet the family's existing skill level(s) in applying them. Attempting to buffer family stress levels and providing supports to improve coping skills are two strategies used by OSLC investigators to improve the family's ability to respond to parenting training. The OSLC knowledge base on parent training and intervention is derived from families of diverse socioeconomic conditions and social and emotional resources. The authors of the FS program attempted to incorporate these values, experiences, and generic strategies into the homeBase component.

The intent of Enhanced First Step research (of which this proposal is a component of) is to improve the home component of the program to more effectively impact the family ecology (i.e., parent/child interactions and relationships, family management practices), and thus the child's behavior. To more dramatically impact a child's behavior in the home setting, a modified version of the Family Check-up (FCU; Dishion \& Stormshak, 2007) is being utilized to (a) assess family values and goals and the strengths and weaknesses of a family's management practices; (b) amplify discrepancies between the child and family's current situation and their goals and values, in order to; (c) resolve ambivalence and motivate the family to develop and implement a change plan to increase 
likelihood of early school success. Results from the first pilot year of the grant were encouraging. 
Appendix B

The Classroom Expectations Checklist

\begin{tabular}{|c|c|}
\hline Universal Principles & $\begin{array}{l}\text { Strengths / Support } \\
\text { Needed }\end{array}$ \\
\hline 1. Have clear expectations & \\
\hline $\begin{array}{l}\text { Expectations are stated positively and } \\
\text { reviewed periodically (e.g. class meetings). } \\
\text { Expectations are used as pre-corrections for } \\
\text { potentially difficult times (transitions, special } \\
\text { events). }\end{array}$ & \\
\hline 2. Teach the expectations & \\
\hline $\begin{array}{l}\text { Expectations are explicitly and directly } \\
\text { taught in the settings in which they occur, } \\
\text { through role-play and demonstrations using } \\
\text { examples and non-examples. }\end{array}$ & \\
\hline 3. Reinforce the expectations & \\
\hline $\begin{array}{l}\text { Expectations are positively reinforced } \\
\text { informally (e.g., personal notes, one to one, } \\
\text { notes home) and formally (e.g., graphs, } \\
\text { charts, activities) to reinforce new skills. }\end{array}$ & \\
\hline $\begin{array}{l}\text { Celebrations to acknowledge complying with } \\
\text { expectations happen on a regular basis. }\end{array}$ & \\
\hline $\begin{array}{l}\text { 4. Minimize attention for minor inappropriate } \\
\text { behaviors }\end{array}$ & \\
\hline $\begin{array}{l}\text { Minor rule infractions are corrected through } \\
\text { reminders; peers and adults systematically } \\
\text { ignore behavior that is annoying or irritating, } \\
\text { but does not violate expectations. }\end{array}$ & \\
\hline 5. Have clear consequences & \\
\hline $\begin{array}{l}\text { A systematic plan exists for the entire class } \\
\text { that consists of a hierarchy of consequences } \\
\text { for when expectations are violated. } \\
\text { Consequences for inappropriate behavior are } \\
\text { individualized (when appropriate). }\end{array}$ & \\
\hline
\end{tabular}




\section{Appendix C}

\section{The First Step Classroom Check-up Action Plan}

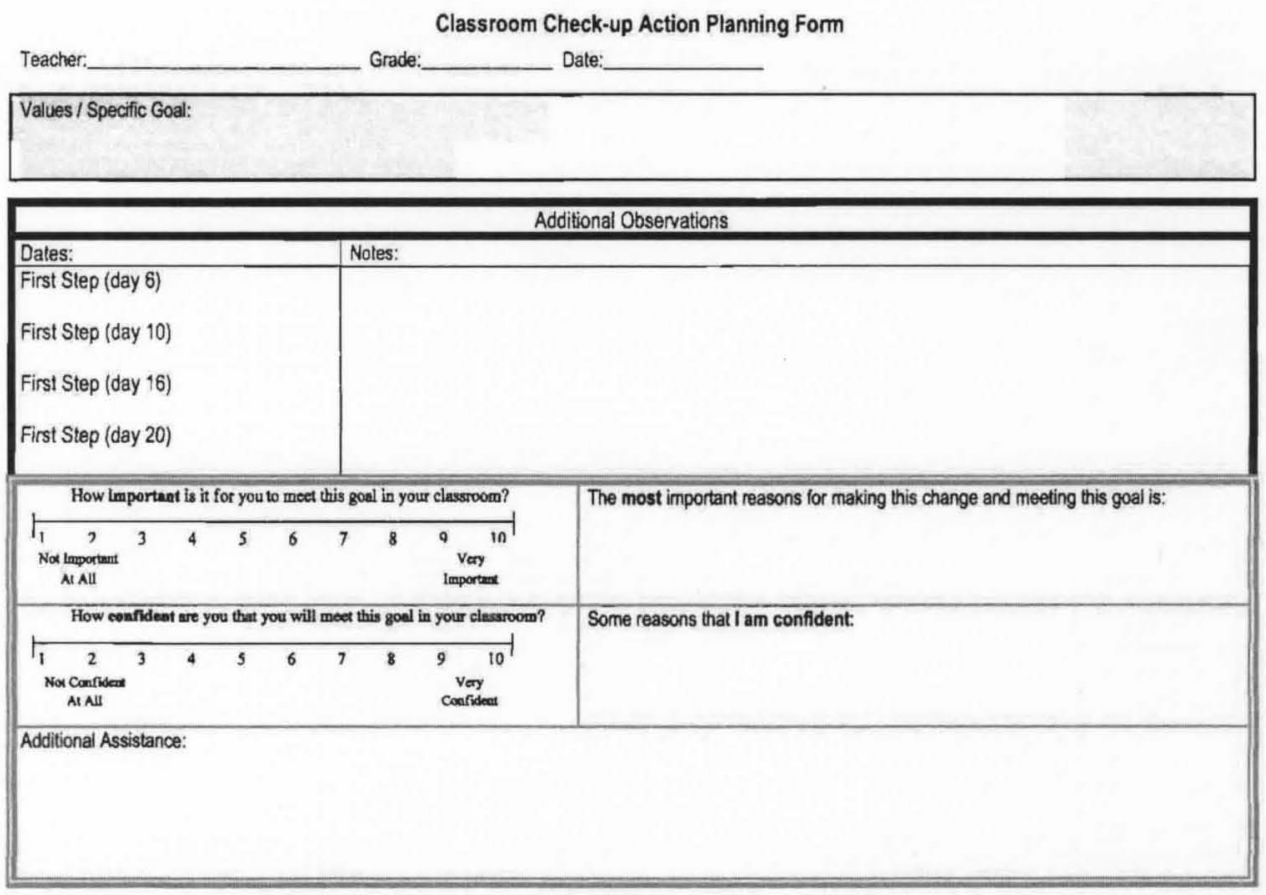


Appendix D

The First Step Classroom Check-up Process Fidelity Checklist

\begin{tabular}{|c|c|}
\hline Steps & Tasks \\
\hline $\begin{array}{l}\text { Information } \\
\text { Gathering }\end{array}$ & $\begin{array}{l}\text { Complete two } 30 \text {-minute observations of teacher } \\
\text { behavior } \\
\text { Create a visual representation of the } \\
\text { observational data }\end{array}$ \\
\hline $\begin{array}{l}\text { Getting to Know } \\
\text { You Interview }\end{array}$ & $\begin{array}{l}\text { Develop and assess a working alliance } \\
\text { Determine teacher's values and goals in relation } \\
\text { to teaching \& education } \\
\text { Listen for and elicit the teacher's experiences } \\
\text { and perceptions of school, teaching, and his or } \\
\text { her use of feedback (positive and negative) in } \\
\text { the classroom } \\
\text { Facilitate teacher self-assessment of the five } \\
\text { universal principles }\end{array}$ \\
\hline $\begin{array}{l}\text { Data Review \& Goal } \\
\text { Setting }\end{array}$ & $\begin{array}{l}\text { Review observational data with the visual } \\
\text { representation } \\
\text { Present menu of options (if needed) } \\
\text { Assess and manage resistance, amplify } \\
\text { discrepancies, cultivate importance, and boost } \\
\text { the teacher's confidence and feelings of self- } \\
\text { efficacy for change } \\
\text { Develop plan of action } \\
\text { Identify goals and target dates for } \\
\text { accomplishment }\end{array}$ \\
\hline $\begin{array}{l}\text { Maintenance } \\
\text { Observations \& } \\
\text { Feedback }\end{array}$ & $\begin{array}{l}\text { Complete four additional observations of teacher } \\
\text { behavior on or near specified days of the First } \\
\text { Step CLASS component (days } 6,10,16,20 \text { ) } \\
\text { Add maintenance observation data to the } \\
\text { original graph and provide it for the teacher } \\
\text { Celebrate progress, revisit menu of options, and } \\
\text { manage resistance to change as necessary }\end{array}$ \\
\hline
\end{tabular}




\section{Appendix E}

\section{Enhanced First Step Fidelity (MI Quality)}

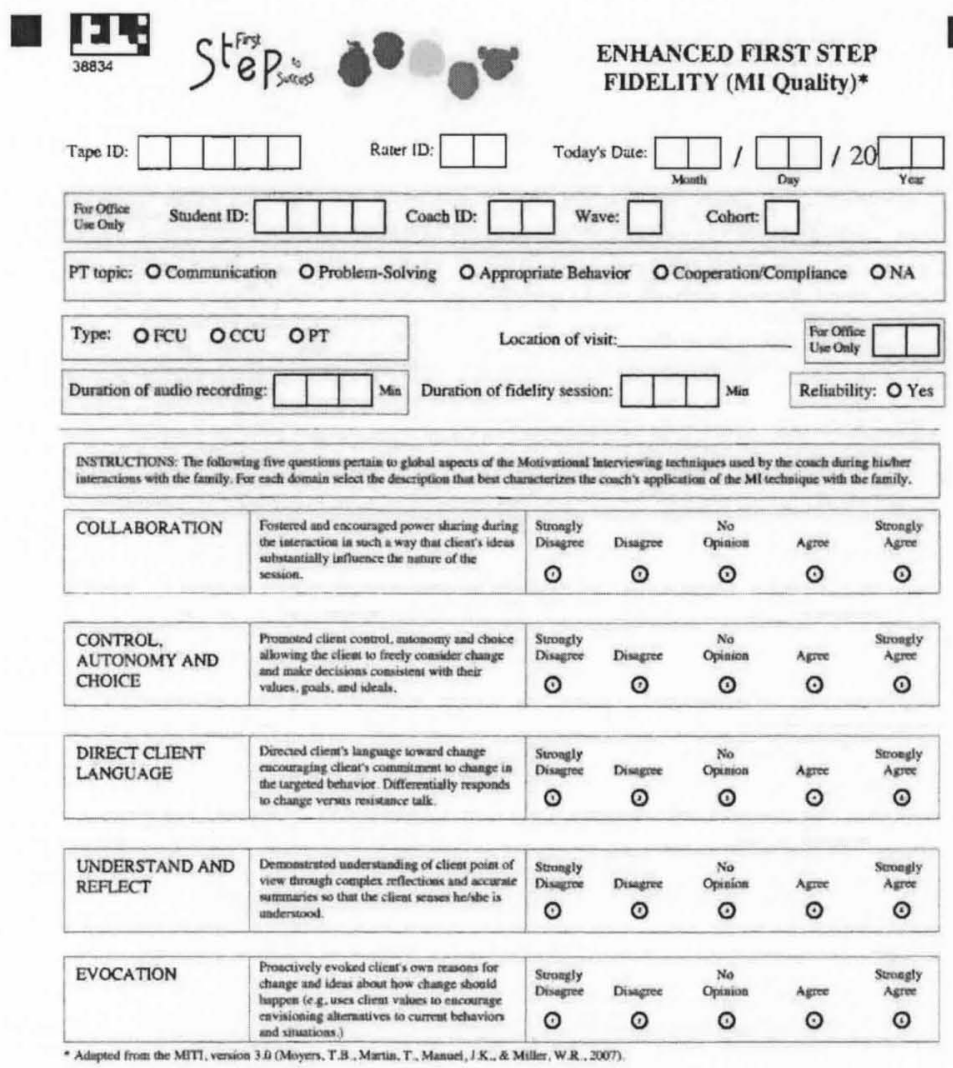

Page 1 of 2 
- $\underset{38834}{\mathbf{E T}}$

\begin{tabular}{|c|c|}
\hline \multicolumn{2}{|l|}{ MIT (IISSH TOTALS) } \\
\hline MmITEMs & MIII RUSH \\
\hline Clond ented questhass (CEQ) & \\
\hline 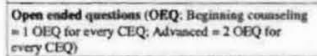 & \\
\hline 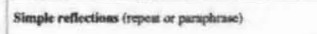 & \\
\hline 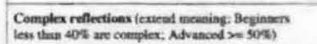 & \\
\hline 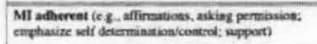 & \\
\hline 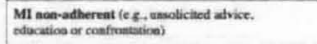 & \\
\hline Inatarnation & \\
\hline
\end{tabular}

Sum tallies frum ubere and enter bedow;

\begin{tabular}{|c|c|}
\hline \multicolumn{2}{|l|}{ MITI (HASH TOTALS) } \\
\hline Mmineses & Mminush \\
\hline Clened ended questions ICEO) & \\
\hline 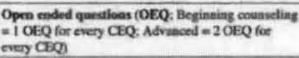 & \\
\hline 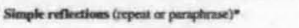 & \\
\hline 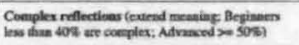 & \\
\hline 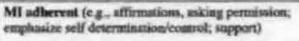 & \\
\hline 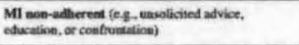 & \\
\hline lafermation & \\
\hline TOTAL. DARNROC BUSH TOTALS & \\
\hline
\end{tabular}

Page 2 of 2 


\section{Appendix F}

The Coach-Teacher Alliance Survey

Int

45210

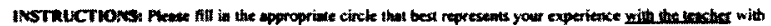
whow you have been workine

\begin{tabular}{|c|c|c|c|c|c|}
\hline & Tener & setdem & Semertimes & Orise & Jimaps \\
\hline 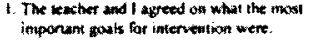 & 0 & 0 & $\odot$ & ○ & 0 \\
\hline 2. The lescher communisuled eflectively. & $\odot$ & (c) & (1) & 0 & 0 \\
\hline $3 \mathrm{Th}$ e teacher and I Irused one anditer. & 0 & 0 & ( ) & 0 & 0 \\
\hline 4. The wachet was appowchable & 0 & 0 & 0 & 0 & ( ) \\
\hline 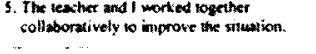 & $\odot$ & ○ & 0 & 0 & 0 \\
\hline $\begin{array}{l}\text { 6. The teacther followed through with } \\
\text { commitments and responsititities. }\end{array}$ & 0 & 0 & 0 & 0 & (c) \\
\hline 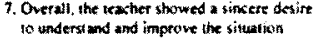 & 0 & 0 & 0 & 0 & (1) \\
\hline $\begin{array}{l}\text { 8. The limse spent werting with the teecher } \\
\text { wax eflective and productive }\end{array}$ & $\odot$ & 0 & O & 0 & $\odot$ \\
\hline
\end{tabular}

Do you tove any cominents or feodhek atoul youl work with inis lewither?
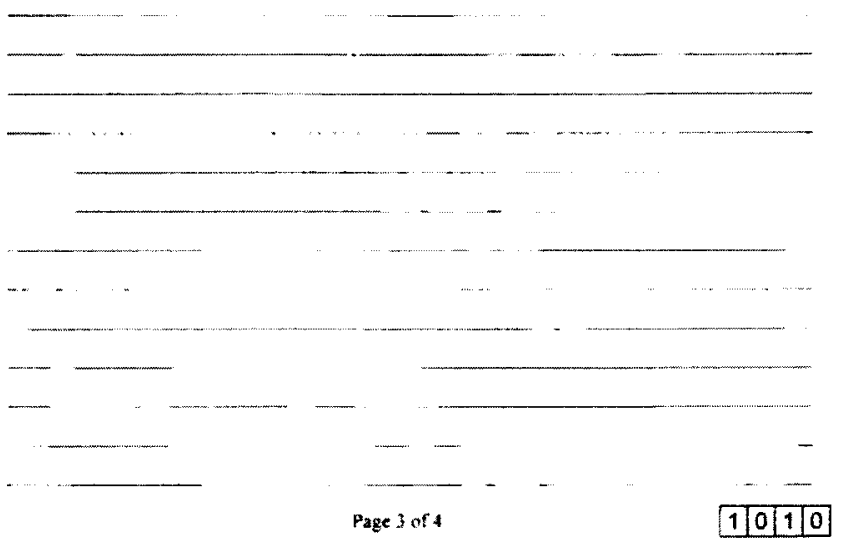
Eir

INSTRUCTIONS: Please fill in the appropriate circle that best represents your experitence with the coact with whom you have been workin

\begin{tabular}{|c|c|c|c|c|c|}
\hline & Never & Seldom & Sometimes & Othen & Always \\
\hline $\begin{array}{l}\text { 1. The coach and I agreed on what the roost } \\
\text { important goals for intervention were. }\end{array}$ & 0 & $\odot$ & $\odot$ & $\odot$ & $\odot$ \\
\hline 2. The coasch conmunicat od effectively. & 0 & ○ & 0 & $\odot$ & ○ \\
\hline 3. The coach and I trustod one another. & 0 & 0 & 0 & $\circ$ & $\odot$ \\
\hline 4 The coasch was approuchable. & $\circ$ & o & 0 & $\odot$ & $\odot$ \\
\hline $\begin{array}{l}\text { 5. The coach and I worked together } \\
\text { collaboratively to improve the stuation. }\end{array}$ & 0 & 0 & 0 & $\odot$ & $\odot$ \\
\hline $\begin{array}{l}\text { 6. The coach followed through with } \\
\text { commictoents and responsibilities. }\end{array}$ & 0 & () & 0 & $\odot$ & $\odot$ \\
\hline $\begin{array}{l}\text { 7. Overall, the coosh showed a sincare desire } \\
\text { to understand and inprove the sauation. }\end{array}$ & 0 & $\odot$ & ० & $\odot$ & $\odot$ \\
\hline $\begin{array}{l}\text { 8. The tirne spent working with the coach was } \\
\text { effetive and productive. }\end{array}$ & $\odot$ & $\odot$ & $\odot$ & $\odot$ & $\odot$ \\
\hline
\end{tabular}

Do you have any corments or feodhock about your work with his coach?

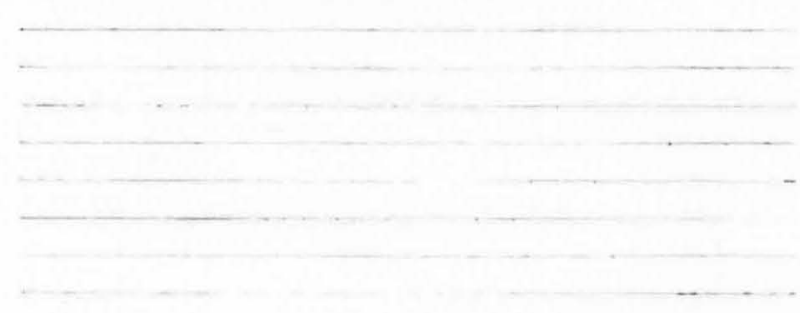

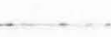

Page 3 of 4

1.0001 


\section{Appendix G}

The Enhanced First Step CLASS Component Fidelity Checklist

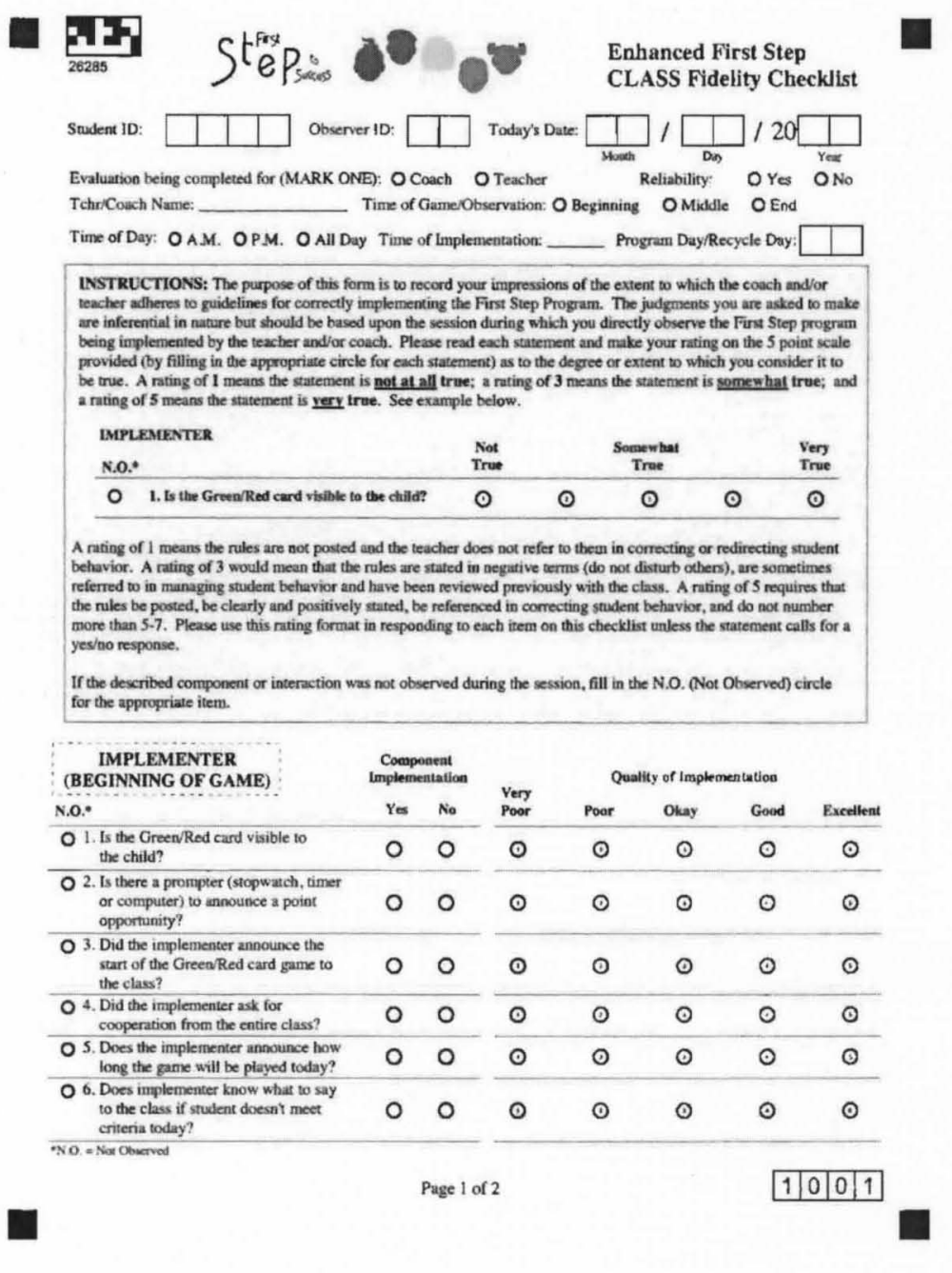




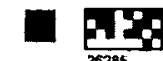

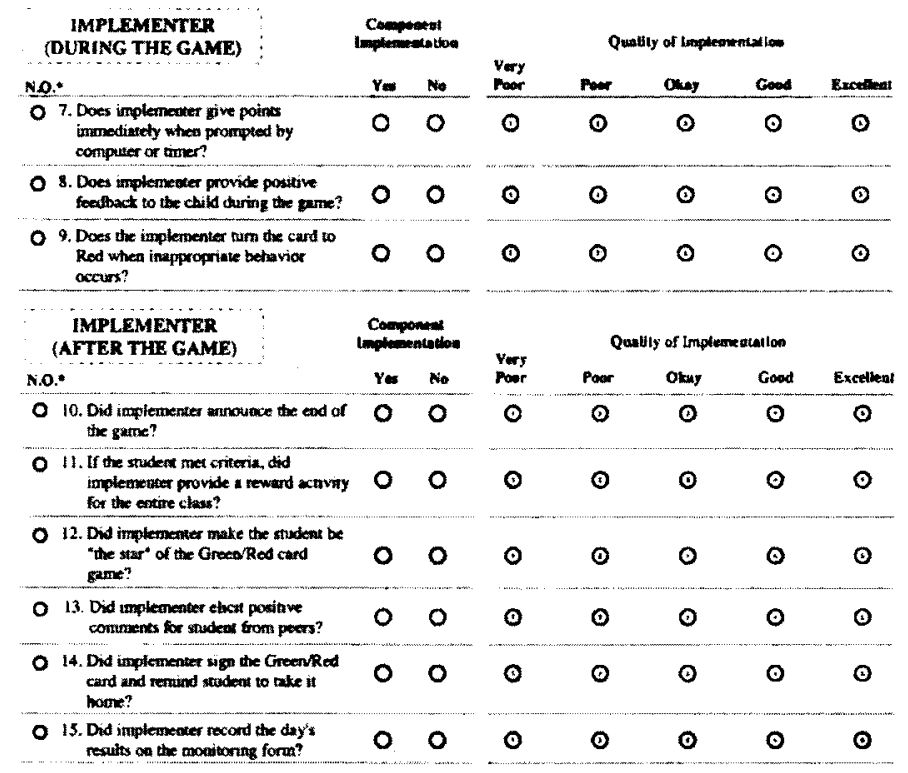$$
\text { -... }
$$

PEERS

\begin{tabular}{|c|c|c|c|c|c|c|}
\hline \multirow{2}{*}{\multicolumn{2}{|c|}{ 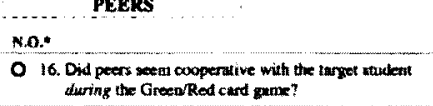 }} & \multirow{2}{*}{$\underset{0}{\operatorname{Nox}}$} & \multicolumn{3}{|c|}{ som } & \multirow{2}{*}{$\begin{array}{c}V_{w r} \\
T=0 \\
0\end{array}$} \\
\hline & & & 0 & $\odot$ & $\odot$ & \\
\hline 0 & 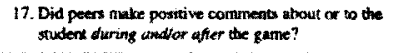 & $\odot$ & (c) & $\odot$ & 0 & 0 \\
\hline 0 & 18. Did poer seem to enjay the rewasd aclivity? & 0 & (1) & 0 & ( & $\odot$ \\
\hline 0 & 19. Do peers appear supportive of the studem? & ○ & 0 & 0 & $\odot$ & o \\
\hline & 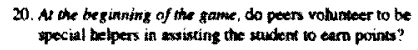 & $\mathbf{0}$ & 0 & $\odot$ & $\odot$ & ○ \\
\hline
\end{tabular}
NO. a Nax Omered 


\section{Appendix $\mathrm{H}$}

The Enhanced First Step to Success CLASS Monitoring Log

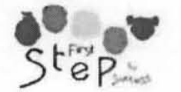

\begin{tabular}{|c|c|c|c|c|c|c|c|c|}
\hline \multicolumn{7}{|c|}{ Student ID Number: } & \multicolumn{2}{|l|}{ Coach's Name: } \\
\hline \multicolumn{7}{|c|}{ Teacher Name: } & \multicolumn{2}{|l|}{ Scbool: } \\
\hline \multicolumn{2}{|c|}{ Daps } & \multicolumn{3}{|c|}{ Potats } & \multicolumn{2}{|c|}{ Winnet } & \multirow{2}{*}{ Reseserd hativing } & \multirow{2}{*}{ 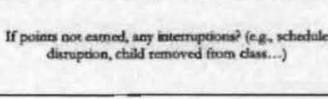 } \\
\hline Dase & $\begin{array}{l}\text { Program Day } \\
\text { from semmary } \\
\text { chary }\end{array}$ & Pousbible & Necoded & Eamed & Yet & No & & \\
\hline & & & & & & & & \\
\hline & & & & & & & & \\
\hline & & & & & & & & \\
\hline & & & & & & & & \\
\hline & & & & & & & & \\
\hline & & & & & & & & \\
\hline & & & & & & & & \\
\hline & & & & & & & & \\
\hline
\end{tabular}




\section{Appendix I}

The Student-Teacher Relationship Scale (short form)

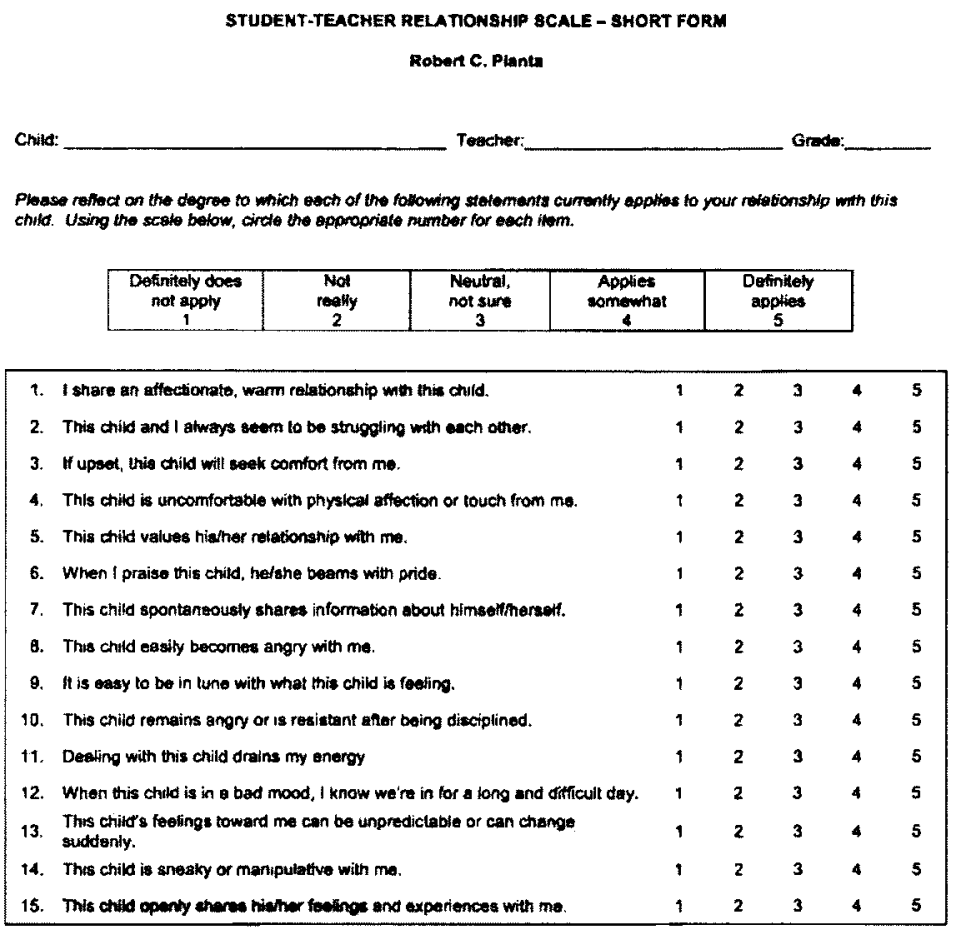

- 1992 Pienta, University af Virginia. 


\section{Appendix J}

\section{Social Validity Questionnaires}

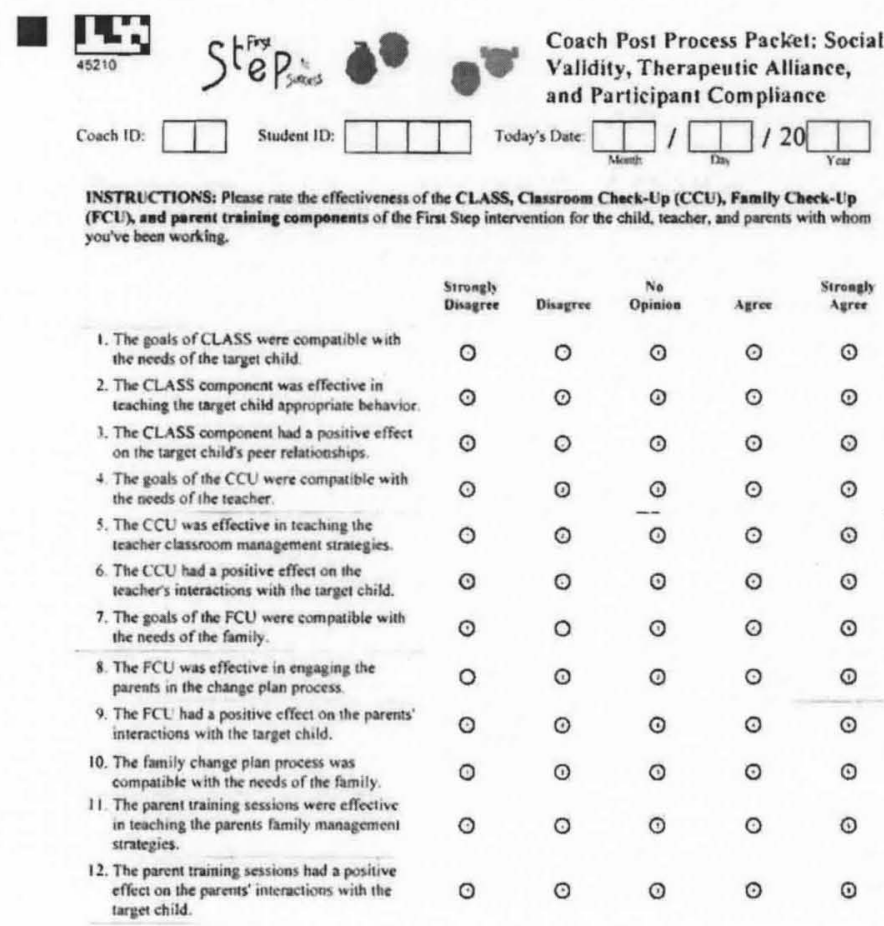

Page 1 of 4 
I I I I

INSTRUCTIONS: Plonse rate your antisfoction with the Chassruom Cbeck-up (CCU) eomponent of the Firs Step intervention by filling in the approprime cincle for each question.

The $\mathrm{CCU}$ component iovolves direet observation and feesteck on positive and nepative satements made by the

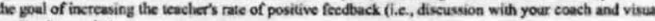
graphs reprexenting the results)

\begin{tabular}{|c|c|c|c|c|c|}
\hline & $\begin{array}{l}\text { Strangly } \\
\text { Disagree }\end{array}$ & Disagree & $\begin{array}{c}\text { No } \\
\text { Opinitos }\end{array}$ & Agree & $\begin{array}{l}\text { Strongly } \\
\text { Agrox }\end{array}$ \\
\hline $\begin{array}{l}\text { 1. The goal of the CCU fit weli with my groals } \\
\text { to mprowe classroom behavioc. }\end{array}$ & 0 & 0 & 0 & 0 & ○ \\
\hline $\begin{array}{l}\text { 2. The goal of the } \mathrm{CCU} \text { was cornpatible with } \\
\text { nyy needs in the classroora. }\end{array}$ & 0 & 0 & ○ & 0 & ○ \\
\hline $\begin{array}{l}\text { 3. The CCU was easy, as comprared to other } \\
\text { profestional developmeat opportuntice } \\
\text { thave experiensed. }\end{array}$ & (1) & O & 0 & 0 & $\odot$ \\
\hline 4. The CCU tid tor take much of my time. & 0 & ○ & $\odot$ & $\odot$ & (1) \\
\hline $\begin{array}{l}\text { 5. The CCU did not interfere with my other } \\
\text { Leaching aetivitiesiresponsibilatics. }\end{array}$ & 0 & 0 & (0) & $\odot$ & $\odot$ \\
\hline $\begin{array}{l}\text { 6. The CCU was effective in changing my } \\
\text { use of feodback with studenas. }\end{array}$ & 0 & 0 & $\odot$ & ○ & $\odot$ \\
\hline $\begin{array}{l}\text { 7. I an satsiffod with the change in my behavios } \\
\text { lowards my gudents. }\end{array}$ & $\circ$ & $\odot$ & $\odot$ & 0 & $\odot$ \\
\hline $\begin{array}{l}\text { 8. The CCUI had a positive effeet on my } \\
\text { relationship with the target chuld }\end{array}$ & ○ & 0 & $\odot$ & $\odot$ & ○ \\
\hline $\begin{array}{l}\text { 9. I was satisfied with the on-going supporthelp i } \\
\text { received from the coach durng the CCU. }\end{array}$ & 0 & 0 & 0 & $\odot$ & $\odot$ \\
\hline $\begin{array}{l}\text { 10. } 1 \text { would recommend the } \mathrm{CO} \text { to other } \\
\text { teachern. }\end{array}$ & $\odot$ & 0 & $\odot$ & $\odot$ & 0 \\
\hline
\end{tabular}

Page 2 of 4 


\section{Appendix K}

Focus group interview questions

\section{Goals / Importance:}

- One focus of First Step Classroom Check-up is to highlight the importance of a teacher's use of positive and negative feedback to children. Tell me a little about the extent to which you believe this is important and why?

- To what extent were you motivated towards change after the feedback session and review of observational data? [prompt for why they found it motivating or not motivating]

- If you set any specific personal goals for yourself as a result of this feedback session, what were they and why did you select these over others you may have considered?

\section{Procedures:}

- If you discussed the five universal principles on which the First Step intervention is based, in what ways did you find this useful? How could we improve the usefulness this discussion?

- Please provide some feedback on the length and time demands associated with the meeting and data review required for the Classroom Check-up in the context of your classroom. [Based on response, circle one of the following a) Require too much, b) required the right amount of time, or c) did not require time\} 
- Feedback session is the meeting with your coach after the first set of observations is complete. Tell me what this experience was like for you? (prompt for how/if it was motivational or a-motivational, and how it was similar or to other professional development activities they have participated in.]

- After the feedback session, did you find the follow-up observations useful? If so, how?

- How did the follow-up observations support your personal goal(s)?

- Tell us a little about your experiences with the visual graphs of your use of positive and negative feedback support your personal goals? [prompt for how the graphs could be made or utilized better?]

\section{Outcomes: (Prompt $=$ Probe for more detail with outcome related answers, specific}

\section{detail and examples are greatly appreciated.)}

- In what ways did the Classroom Check-up affect your attitude towards or relationship with the focus child, other children, or the classroom climate in general?

- Can you share a little about whether or not you believe the meetings with your coach, goal setting, and the visual displays of positive vs. negative feedback were effective in changing your behavior?

- Tell me how the intervention was or was not effective in changing the focus child's behavior in the classroom?

- What did you enjoy most about the Classroom Check-up? 
- What did you enjoy least about the Classroom Check-up?

- We are considering changing the observations to include only the focus child, what are your thoughts about this change?

- We are also considering tracking verbal and non-verbal feedback (i.e., thumbs up, winks), what are your thoughts about this possible change?

- In your opinion, what else could, or should, be done to improve the success of the Classroom Check-up? 
Appendix L

Case-level outcome results

Table N1. Case Level MI Implementation Integrity (Quality)

\begin{tabular}{|c|c|c|c|c|c|}
\hline $\begin{array}{l}\text { Teach } \\
\text { ID }\end{array}$ & $\begin{array}{l}\text { Global Spirit } \\
\text { Composite }\end{array}$ & $\begin{array}{l}\text { Reflections: } \\
\text { Questions Ratio }\end{array}$ & $\begin{array}{l}\text { Percent Open } \\
\text { Questions }\end{array}$ & $\begin{array}{l}\text { Percent } \\
\text { Complex } \\
\text { Reflections }\end{array}$ & $\begin{array}{l}\text { Percent MI } \\
\text { Adherent }\end{array}$ \\
\hline 5021 & $4.00(C)$ & $1.00(\mathrm{P})$ & 0.33 & $0.67(\mathrm{C})$ & $1.00(\mathrm{C})$ \\
\hline 5022 & $4.33(\mathrm{C})$ & $1.31(\mathrm{P})$ & $0.54(\mathrm{P})$ & $0.53(\mathrm{C})$ & $1.00(\mathrm{C})$ \\
\hline 5028 & $4.00(C)$ & $1.70(\mathrm{P})$ & 0.40 & $0.53(\mathrm{C})$ & $1.00(\mathrm{C})$ \\
\hline 5029 & $4.00(\mathrm{C})$ & 0.60 & 0.20 & 0.33 & $1.00(\mathrm{C})$ \\
\hline 5030 & $4.67(\mathrm{C})$ & 0.86 & $0.71(\mathrm{C})$ & 0.33 & $1.00(\mathrm{C})$ \\
\hline 5031 & $4.67(\mathrm{C})$ & 0.38 & $0.50(\mathrm{P})$ & 0.33 & $1.00(\mathrm{C})$ \\
\hline 5033 & $4.33(\mathrm{C})$ & $2.00(\mathrm{C})$ & $1.00(\mathrm{C})$ & $1.00(\mathrm{C})$ & $1.00(\mathrm{C})$ \\
\hline 5038 & $4.33(\mathrm{C})$ & 0.58 & 0.42 & 0.14 & $1.00(\mathrm{C})$ \\
\hline 5039 & $3.67(\mathrm{P})$ & 0.27 & $0.53(\mathrm{P})$ & 0.25 & $1.00(\mathrm{C})$ \\
\hline 5042 & $4.00(\mathrm{C})$ & 0.24 & $0.53(\mathrm{P})$ & 0.25 & $0.91(\mathrm{P})$ \\
\hline 5043 & $3.67(\mathrm{P})$ & $1.00(\mathrm{P})$ & $0.50(\mathrm{P})$ & 0.33 & $0.71(\mathrm{P})$ \\
\hline $5045^{*}$ & -- & -- & - & - & -- \\
\hline$M(S D)$ & $4.17(.33)(\mathrm{C})$ & $.82(.36)$ & $.47(.03)$ & $.40(.10)(P)$ & $.97(.05)(P)$ \\
\hline
\end{tabular}

MITI Summary Score Competency Thresholds; $\mathrm{C}=$ Competency (highest level); $\mathrm{P}=$ Proficiency.

*Teacher 5045 did not provide permission for audio recording, thus MITI summary scores are unavailable. 
Table N2. Coach-Teacher Alliance Survey Results (Wave 2 Only).

Data available for Wave 2 participants only. Scores represent average of eight alliance survey questions, rated on a Likert-type scale from 1(never) to 5 (always).

\begin{tabular}{llll}
\hline Child ID & $\begin{array}{l}\text { Coach Perception of } \\
\text { Alliance with Teacher } \\
M(S D)\end{array}$ & $\begin{array}{l}\text { Teacher Perception of } \\
\text { Alliance with Coach } \\
M(S D)\end{array}$ & Classification \\
\hline 1100 & $4.00(.00)$ & $5.00(.00)$ & Strong \\
1106 & $4.00(.53)$ & $5.00(.00)$ & Strong \\
1109 & $4.25(.46)$ & $5.00(.00)$ & Strong \\
1163 & $5.00(.00)$ & $4.88(.35)$ & Strong \\
1164 & $4.75(.46)$ & $5.00(.00)$ & Strong \\
1170 & $4.75(.46)$ & $5.00(.00)$ & Strong \\
1173 & $4.00(.53)$ & $5.00(.00)$ & Strong \\
1181 & $4.38(1.06)$ & $4.88(.35)$ & Strong \\
\hline $\boldsymbol{M}$ & $\mathbf{4 . 3 9 ( . 2 4 )}$ & $\mathbf{4 . 9 7 ( . 0 9 )}$ & Strong \\
\hline
\end{tabular}

Table N3. Student-Teacher Relationship Subscale; Conflict.

\begin{tabular}{|c|c|c|c|c|c|}
\hline $\begin{array}{l}\text { Teacher } \\
\text { ID }\end{array}$ & $\begin{array}{l}\text { Child } \\
\text { ID }\end{array}$ & $\begin{array}{l}\text { Baseline } \\
\text { Total score } \\
\text { (percentile) }\end{array}$ & $\begin{array}{l}\text { Post } \\
\text { Total score } \\
\text { (percentile) }\end{array}$ & $\begin{array}{l}\text { RCI } \\
\text { Statistic }\end{array}$ & Classification \\
\hline 5030 & 1100 & $41(92)$ & $20(50)$ & 5.859 & Responder \\
\hline 5028 & 1106 & $28(79)$ & $31(92)$ & -0.84 & Non \\
\hline 5029 & 1109 & $47(87)$ & 37 (3) & 2.79 & Responder \\
\hline 5022 & 1117 & $34(70)$ & $26(40)$ & 2.232 & Responder \\
\hline 5031 & 1123 & $43(96)$ & $38(92)$ & 1.395 & Non \\
\hline 5033 & 1128 & $42(93)$ & $39(90)$ & 0.837 & Non \\
\hline 5021 & 1144 & $26(70)$ & $22(60)$ & 1.116 & Non \\
\hline 5038 & 1163 & $51(75)$ & $56(99)$ & -1.40 & Non \\
\hline 5039 & 1164 & $31(79)$ & $35(84)$ & -1.12 & Non \\
\hline 5042 & 1170 & $27(72)$ & $15(30)$ & 3.348 & Responder \\
\hline 5043 & 1173 & $35(84)$ & $59(99)$ & -6.7 & Non \\
\hline 5045 & 1181 & $50(99)$ & $34(84)$ & 4.464 & Responder \\
\hline$M(S D)$ & & $37.92(8.96)$ & $34.33(13.26)$ & & \\
\hline
\end{tabular}

STRS critical cut off point for Conflict $>75^{\text {th }}$ percentile.

Bold $=$ RCI statistic $>1.96$ or $>-1.96$; Negative RCI statistic $=$ increased conflict. 
Table N4. Student-Teacher Relationship Subscale; Closeness.

\begin{tabular}{|c|c|c|c|c|c|}
\hline $\begin{array}{l}\text { Teacher } \\
\text { ID }\end{array}$ & Child ID & $\begin{array}{l}\text { Baseline } \\
\text { Total score } \\
\text { (percentile) }\end{array}$ & $\begin{array}{l}\text { Post } \\
\text { Total score } \\
\text { (percentile) }\end{array}$ & $\begin{array}{l}\text { RCI } \\
\text { Statistic }\end{array}$ & Classification \\
\hline 5030 & 1100 & $47(60)$ & $54(95)$ & 1.32 & Non \\
\hline 5028 & 1106 & $53(92)$ & $54(95)$ & 0.19 & Non \\
\hline 5029 & 1109 & $26(3)$ & $35(15)$ & 1.7 & Non \\
\hline 5022 & 1117 & $43(40)$ & $44(45)$ & 0.19 & Non \\
\hline 5031 & 1123 & $32(5)$ & $41(20)$ & 1.7 & Non \\
\hline 5033 & 1128 & $23(1)$ & $40(27)$ & 3.21 & Responder \\
\hline 5021 & 1144 & $50(80)$ & $40(27)$ & -1.89 & Non \\
\hline 5038 & 1163 & $22(1)$ & $13(1)$ & -1.70 & Non \\
\hline 5039 & 1164 & $39(25)$ & $36(19)$ & -0.57 & Non \\
\hline 5042 & 1170 & $48(70)$ & $52(90)$ & 0.75 & Non \\
\hline 5043 & 1173 & $44(45)$ & $34(12)$ & -1.89 & Non \\
\hline 5045 & 1181 & $34(12)$ & $44(45)$ & 1.89 & Non \\
\hline$\overline{M(S D)}$ & & $38.42(10.82)$ & $40.58(11.16)$ & & \\
\hline
\end{tabular}

STRS critical cut off point for Closeness $<25^{\text {th }}$ percentile.

Bold $=$ RCI statistic $>1.96$ or $<-1.96$; Negative RCI statistic $=$ decreased closeness.

Table N5 Teacher Behavior (OTB)

\begin{tabular}{lllll}
\hline $\begin{array}{l}\text { Teacher } \\
\text { ID }\end{array}$ & $\begin{array}{l}\text { Child } \\
\text { ID }\end{array}$ & $\begin{array}{l}\text { Baseline Unit } \\
\text { Rate }\end{array}$ & $\begin{array}{l}\text { Post Unit } \\
\text { Rate }\end{array}$ & Classification \\
\hline 5030 & 1100 & $\mathbf{3 . 8 7}$ & 2.12 & Non \\
5028 & 1106 & 1.66 & $\mathbf{3 . 1 6}$ & Responder \\
5029 & 1109 & 2.66 & $\mathbf{5 . 6 6}$ & Responder \\
5022 & 1117 & 0.2 & 1.8 & Non \\
5031 & 1123 & 1.29 & $\mathbf{5 . 8 8}$ & Responder \\
5033 & 1128 & 0.38 & 2.84 & Non \\
5021 & 1144 & 1.18 & $\mathbf{3 . 4 4}$ & Responder \\
5038 & 1163 & $\mathbf{4 . 4 4}$ & $\mathbf{2 0 . 0}$ & Responder \\
5039 & 1164 & $\mathbf{3 . 7 2}$ & $\mathbf{5 . 0 0}$ & Responder \\
5042 & 1170 & 0.88 & 2.18 & Non \\
5043 & 1173 & 1.26 & 1.70 & Non \\
5045 & 1181 & 2.33 & $\mathbf{4 . 4 2}$ & Responder \\
\hline
\end{tabular}

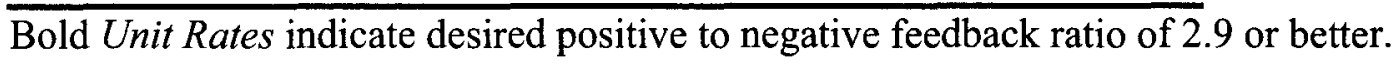


Table N6. CLASS Component Teacher Implementation Fidelity.

\begin{tabular}{lll}
\hline Child ID & $\begin{array}{l}\text { Implementation } \\
\text { Adherence }\end{array}$ & $\begin{array}{l}\text { Implementation } \\
\text { Quality (SD) }\end{array}$ \\
\hline 1100 & 0.97 & 0.93 \\
1106 & 0.95 & 0.89 \\
1109 & 1.00 & 0.88 \\
1117 & 0.96 & 0.82 \\
1123 & 0.87 & 0.83 \\
1128 & 0.9 & 0.76 \\
1144 & 0.96 & 0.89 \\
1163 & 1.00 & 0.89 \\
1164 & 0.93 & 0.68 \\
1170 & 0.97 & 0.75 \\
1173 & 0.93 & 0.84 \\
1181 & 1.00 & 0.8 \\
M Rating (SD) & $.95(.04)$ & $.83(.07)$ \\
\hline
\end{tabular}

The First Step Classroom Check-up Analysis Classification System; .80 and above $=$ adequate, .90 and above $=$ excellent.

Table N7. Intervention Dosage and Student Compliance.

\begin{tabular}{lrr}
\hline Child ID & $\begin{array}{r}\text { Intervention } \\
\text { Dosage }\end{array}$ & $\begin{array}{r}\text { Student } \\
\text { Compliance }\end{array}$ \\
\hline 1100 & .97 & .97 \\
1106 & .56 & .77 \\
1109 & .87 & .87 \\
1117 & .97 & .97 \\
1123 & .93 & .93 \\
1128 & .77 & .77 \\
1144 & .50 & .53 \\
1163 & .86 & .90 \\
1164 & .77 & .95 \\
$1170^{*}$ & -- & - \\
1173 & .43 & .56 \\
1181 & .60 & .72 \\
M (SD) & $.75(.19)$ & $.81(.16)$ \\
\hline
\end{tabular}

*First Step Monitoring Log was unavailable for Focus student 1170. 
Appendix M

Recording Procedures for the Observation of Teacher Behavior Recoding procedures and form for the observation of teacher behavior In order to sample an appropriate representation of a teacher's use of praise and reprimands, the initial set of observations includes two thirty-minute observations totaling 60 minutes. Both 30 -minute observations occur during a structured activity time in the classroom. Follow-up observations (four total) are 15 minutes in length.

Structured activities are defined as opportunities during a classroom period when the teacher's expectations are clear and there is an academic focus. Typical structured activities during which a classroom observation might be completed in the primary grades include settings when the teacher is in charge of the instruction, for example, circle time, small group lessons, or direct instructional situations.

A separate Observation of Teacher Behavior recording form is utilized for each observation. To conduct an observation of teacher behavior, the observer needs a timer, and the recording form. This form is designed for recording the nature of the social interactive behavior engaged in by the focus teacher. This information is coded, during the observation, in the large rectangular boxes near the bottom of the recording form. The top two boxes are labeled Praise (p) and Reprimands (R) respectively. These two boxes are also labeled at the top of each column with Target, Peer, or Class. These labels are intended to represent the focus child who receives the First Step intervention, any other peer in the classroom, or the entire class. 
During the observation, any instance of specific praise that occurs during the 30 or subsequent 15 -minute observation period should be recorded by simply placing an $\mathrm{S}$ in the appropriate box. Any instance of general praise should be recorded with a G.

Likewise, any instance of reprimand that occurs during the observation period should be recorded by simply placing a tally mark in the appropriate box.

The two boxes at the bottom of the recording form labeled Positive StudentTeacher Interactions (PTSI), and Negative Teacher-Student Interactions (NTSI) are for the recording of any instance of teacher behavior that meet the corresponding definitions. These behaviors can be recorded by simply placing a tally mark in the appropriate box; these recordings are separated by Target and Peer categories only.

Code Category Definitions. In order for observers to differentiate the various teacher behaviors that might be observed the following are definitions should be memorized and referred to often.

Praise. Teacher praise involves neutral to positive forms of teacher behavior directed to a student (or the classroom as a whole) who is behaving appropriately. Praise may involve teacher verbalizations or physical gesture. Praise statements and praise gestures are often intended to encourage \& maintain the student's appropriate behavior or that of a peer or the entire classroom.

Specific Praise (S). Specific praise is coded for verbal praise statements that provide specific behavior oriented feedback to a student (or class as a whole) (e.g., "Class, thank you for remembering the "hands off' rule for hallway behavior!" or "Pam, 
thank you for raising your hand!"). The category specific praise requires teacher verbalization, and is never coded for a praise gestures.

General Praise (G). General praise is coded for praise statements that do not provide specific behavior oriented feedback to a student (or the class as a whole) (e.g., “Good job!" or "Way to go, Steven!"). Praise gestures are always coded as general praise (e.g., a thumbs up, or clapping of hands).

Reprimands. Teacher reprimands are directed toward a student (or the class as a whole) who is behaving inappropriately and are designed to either redirect or terminate inappropriate behavior. They are usually delivered in a negative, disapproving tone and must involve teacher verbalization(s) (e.g., "Stop that!" or "I cannot believe this class is so loud," and "You need to line up immediately.") Table G1 provides examples of each category of praise of reprimands.

Table G1. Code category examples

\begin{tabular}{lll}
\hline Specific Praise & General Praise & Reprimands \\
\hline Thanks for being so quiet! & Great Job! & Stop that! \\
$\begin{array}{l}\text { Terrific! You've completed the } \\
\text { entire list. }\end{array}$ & Seriously good & Shh! \\
You are sitting so quiet, thanks! & Way to go! & $\begin{array}{l}\text { I cannot believe you did that, } \\
\text { please stop. }\end{array}$ \\
Great capitalization, Sara! & Awesome! & Sit down...now! \\
Thanks for raising your hand. & You're great! & $\begin{array}{l}\text { No! } \\
\text { This room looks so clean! Great } \\
\text { Job. }\end{array}$ \\
\hline
\end{tabular}


Positive Teacher-Student Interactions (PTSI). PTSIs involve a reciprocal social exchange between the teacher and student in which a) both the teacher and student make verbal statements and b) the social exchanges are all neutral to positive on the part of both student and teacher.

Negative Teacher-Student Interactions (NTSI). NTSIs are identical to PTSIs with the exception that the social exchanges between teacher \& students are negative in tone, may express disapproval, involve opposition or resistance, and/or communicate anger. Difficult students tend to escalate in these situations and often get into arguments with the teacher that can end in direct non-compliance and even defiance.

\section{The First Step Classroom Check-up Recording Instructions}

Observers should select a period in which the activity of the class meets the definition of a structured activity (as defined above). Using the EFS Classroom Check-up Observation of Teacher Behavior recording form, observers should fill in the child's ID, the date of the observation, and observer codes. The type of observation and the description the activity that best fits can be recorded by filling in the corresponding bullets, or by describing the activity in the space provided. The hour and the minute the observation begins can be recorded alongside "Time Start."

Observe the teacher's behavior continuously for 30 minutes, using a timer to monitor the time carefully, while recording each codable behavior in the appropriate section of the observation form. After exactly 30 minutes, fill in the hour and the minute 
the observation ended alongside "Time Stop," and the length of the session in minutes alongside "Total Time Observed."

A frequency count of the total number of tally marks that were made for the Praise and Reprimand behaviors can be recorded in the small boxes on the bottom right side of the page. These boxes are labeled, \# P: for the praise behaviors and \# R: for reprimands. The same should be completed for the total number of PTSI and NTSI behaviors that were observed. 


\section{Appendix $\mathrm{N}$}

\section{MITI Global Rating Revision Description}

When we first began cxamining the MITI for our use, we asticipated traigiag our own staff to code them. When our metbodologist examined it he folt the existing MTT global ratings inctuded multiple dimensions and werc not mutually exclusive. He also wondered if it would be poesible to modify the form of the stem end response opcions so that the stern represented the ideal (i.e, bigh fidelity on the MTTI) and the response options anchoring all of the items on Likert-type scale (strongly disagree - stroogly agree). Terti Moyers assisted with the languaging of these on a couple of occesions, and agree that our application (i.e, indirecs aervice delivery model and in the context of a psychoeducational intervention) was distioct ejough from whal bas typically been dooc that the existing standarts (i.e., beginner/proficieat) may not be the stadard we use anyway. So, we revised. Below is tabie that compares the original global items with our revised global itcms.

\begin{tabular}{|c|c|c|}
\hline $\begin{array}{l}\text { Rerlied Globul Dirension } \\
\text { end description }{ }^{1}\end{array}$ & MITI-Revised Globel Dimeusion Rexposse Opttons & Notes \\
\hline 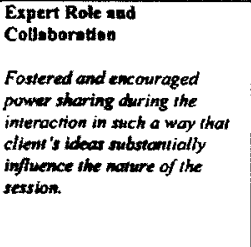 & 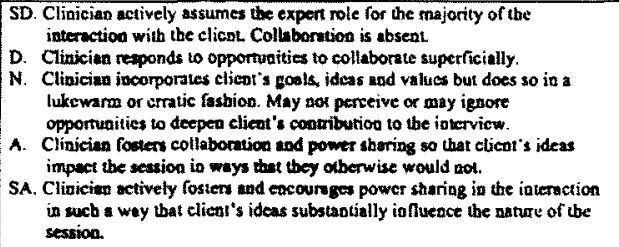 & $\begin{array}{l}\text { The original Mrrn global is } \\
\text { labeled Colluboration. We } \\
\text { did not cbange the coetent } \\
\text { there, asd the uriginal MrTh } \\
\text { responsc options are simply } \\
\text { mapped oolo our revised } \\
\text { stem. We bave retained the } \\
\text { ducl focus oo expert and } \\
\text { collaboration. }\end{array}$ \\
\hline 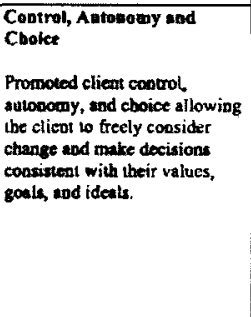 & 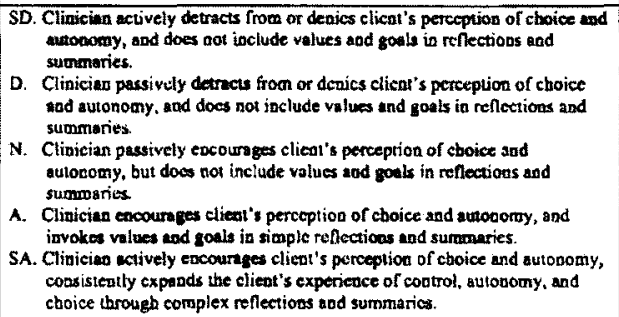 & 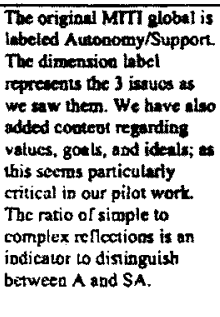 \\
\hline
\end{tabular}

'All hems hove response options ranging from Strongly Aeret to Strondv Olsagree 


\begin{tabular}{|c|c|c|}
\hline $\begin{array}{l}\text { Direct Client Langunge } \\
\text { Direchud client's language } \\
\text { towant chasge through } \\
\text { differential responses to } \\
\text { change and resistance thlk, } \\
\text { eneournging the client's } \\
\text { commitment to change in the } \\
\text { carget bebavior. }\end{array}$ & 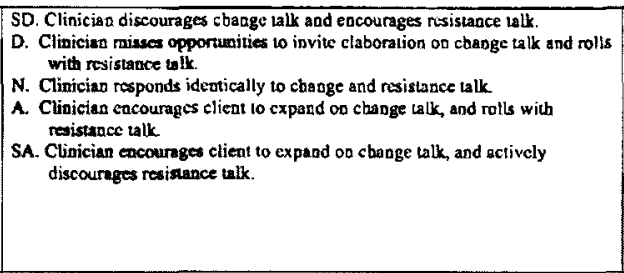 & $\begin{array}{l}\text { The original MITI global is } \\
\text { labeled Directioa. We gre } \\
\text { wondering if ours stould be } \\
\text { Differentially Responds to } \\
\text { Chenge. It was our attemp' } \\
\text { bere to distinguish between } \\
\text { this item and the Evocation } \\
\text { item. Target bebavior for us } \\
\text { are the } 5 \text { ugiversal } \\
\text { principles. Terri indicates a } \\
\text { real interest in the ilem's } \\
\text { functionality. }\end{array}$ \\
\hline $\begin{array}{l}\text { Uaderstand and Reflect } \\
\text { Demonstrated understonding } \\
\text { of client point of view through } \\
\text { complex reflections and } \\
\text { accurate summaries so that the } \\
\text { client senses he/she is } \\
\text { understood. }\end{array}$ & 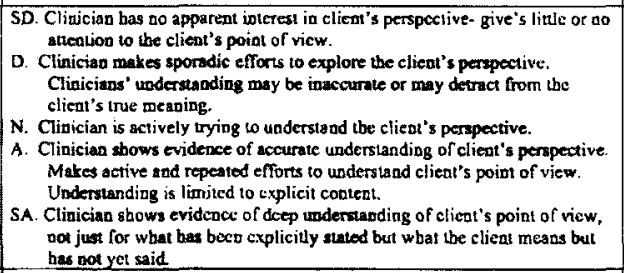 & 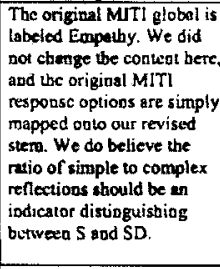 \\
\hline $\begin{array}{l}\text { Evocation } \\
\text { Proactively evoked client's } \\
\text { own reasions for change and } \\
\text { idear about how change } \\
\text { should happen (e.g., uses client } \\
\text { values to encowrage } \\
\text { envisioning ahemastues to } \\
\text { current behaviors and } \\
\text { sirtortions). }\end{array}$ & 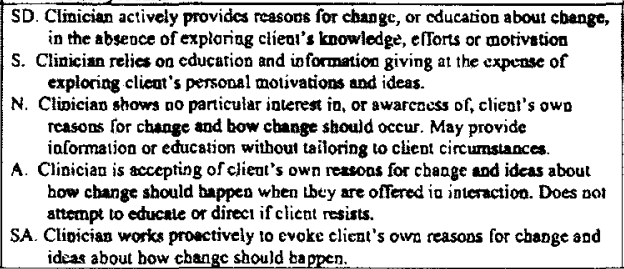 & $\begin{array}{l}\text { We did oot change the } \\
\text { dimeasion tabel or conient } \\
\text { bere, and the original MITI } \\
\text { response options are simply } \\
\text { mapped onto our revised } \\
\text { stcm. }\end{array}$ \\
\hline
\end{tabular}




\section{Appendix O}

\section{The First Step to Success Enhancements Manual Table of Contents}

\section{Table of Contents}

Introduction.

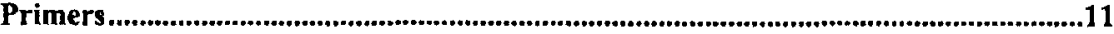

Differential Responding to Change Talk ..............................................................................12

Assessment of Motivation ...........................................................................................................13

Assessment of Working Alliance ...........................................................................................14

Framing Problems within Family Routines and Child Development Context...............15

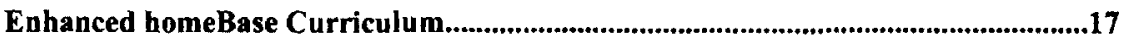

Step 1. Complete Value Discovery and Assessment of Current Practices..............18 Parent Values Discovery Activity and Universal Principles Interview

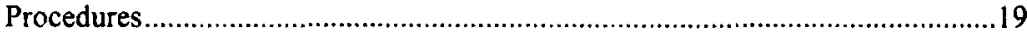

Parent Values Discovery Cards ..............................................................................24

Parent Universal Principles Overview......................................................................27

Parent Universal Principles Interview Guide ......................................................28

Step 2. Collect Current Practices Data ................................................................22

Parent Observation of the Universal Principles Procedures ......................30

Parent Observation of the Universal Principles Coding Form..................32

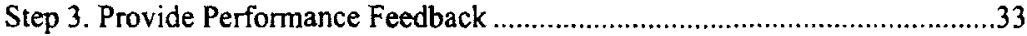

Parent Debriefing Interview Procedures................................................34

Step 4. Provide Parent Extended Consultation, Education, \& Support ..................38

Parent Extended Consultation, Education, \& Support Procedures ............39

Parent Change Plan Worksheet ...................................................................................41

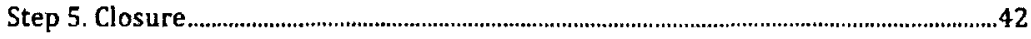

Parent Closure Cover Letter .....................................................................................43

Parent Tip Sheets............................................................................................................44

Enhanced homeBase Troubleshooting Guide ..........................................48

First Step Classroom Check-up Curriculum ................................................................51

Step 1. Conduct Values Discovery and Current Practices Assessment ...................5

Teacher Values Discovery and Universal Principles Interview Procedures

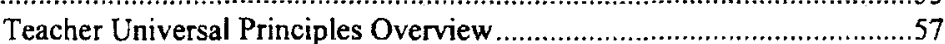

Teacher Universal Principles Interview Guide .......................................58

Step 2. Collect Current practices Data ............................................................59

Teacher Observation of the Universal Principles Procedures...................60

Teacher Observation of the Universal Principles Coding Form ...............62

Step 3. Provide Performance Feedback ....................................................63

Teacher Debriefing Interview Procedures .............................................64

Step 4. Teacher Extended Consultation, Education, \& Support ..........................67

Teacher Extended Consultation, Education, \& Support Procedures .........68 
Teacher Change Plan Worksheet...

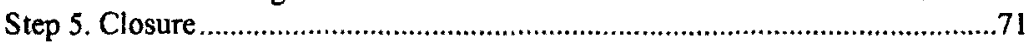

CLASS Modifications for Children Requiring Tertiary Level Support ................................72

Modifications for All Children Identified with Tertiary Level Challenges ......................73

Functional Behavior Assessment Packet .............................................75

Modifications Related to the Individual Characteristics of the Child ...............................82

Modifications Related to Teacher Fidelity .....................................................83

Modifications Related to Parent Fidelity ........................................................8 83

CLASS Progress Monitoring Procedure...........................................84

CLASS Progress Monitoring Chart ...................................................85

Special Considerations for Restrictive Settings ...............................................................87

CLASS Modifications Troubleshooting Guide and Checklist...........................89 


\section{CURRICULUM VITA \\ Jonathan Scott Lee 1640 Bonnycastle Ave Louisville, KY 40205 502.459.5620 Home fourjays@bellsouth.net}

\section{Education}

2012 Ph. D., University of Louisville, Louisville, KY

Curriculum and Instruction with a specialty in Teaching and Learning; Early Childhood and Family Studies. Dissertation Title: Teacher motivation as an enhancement to the First Step to Success early intervention program for Children with Tertiary Level Behavior Challenges.

1992 M.A., University of Northern Colorado, Greeley, CO Early Childhood Special Education

1987 B.A., University of Northern Colorado, Greeley, CO Special Education, K-12

\section{Academic Appointments}

2012-present Assistant Professor. University of Cincinnati, College of Education, Criminal Justice, and Human Services.

2009-2012 Research Faculty. University of Louisville, Kent School of Social Work.

2007-2009 Instructor. University of Louisville, College of Education and Human Development.

2003-2007 Assistant Professor. Bellarmine University, College of Education.

\section{Professional Experience}

2000-2003 Senior Training Specialist, Project Manager for Design and Development. National Center for Family Literacy, Louisville, KY 
1994-2000 Coordinator for Early Childhood and Family Literacy. Windsor RE-4 School Dist. Windsor, CO.

1993-1994 Coordinator Early Childhood Programs. Greeley School Dist. 6. Greeley, CO. 1990-1993 Early Childhood Special Educator. Windsor RE-4 School Dist. Windsor, CO.

1987-1990 Early Childhood Special Educator. Centennial Developmental Services Inc. Evans, CO.

\section{Peer-Reviewed Publications}

Frey, A.J., Cloud, R.N., Lee, J., Small, J. Seeley, J., Feil, E., Walker, H.W., \& Golly, A. (2011). The promise of motivational interviewing in school mental health. School Mental Health, 3, 1-12.

McCart, A., Lee, J., Frey, A.J., Wolf, N. Choi, J.H., \& Haynes, H. (2010).

Response to intervention in early childhood centers: A multitiered approach promoting family engagement. Early Childhood Services, 4, 87-104.

Darling, S., \& Lee, J. (2003) Linking parents to reading instruction. The Reading Teacher, 57, 382-384.

\section{Publications Under Development}

Frey, A., Lee, J., Seeley, J. Walker, H. \& Small, J. The Motivational Interviewing Flowchart: A navigational map for teaching and learning. Under review, Journal of Educational \& Psychological Consultation. February 2012.

Lee, J., Ratcliffe, P., Rutledge, A., Frey, A.J., Walker, H.M., \& Seeley, J. Lessons learned in infusing Motivational Interviewing into an early intervention program for children with challenging behavior. To be submitted to the Journal of School Mental Health.

Frey, A., Lee, J., Small, J., Seeley, J., Walker, H., \& Feil, E. Transporting Motivational Interviewing to school settings. To be submitted to the Journal of Applied Social Psychology.

Lee, J., Frey, A., J., Seeley, J. Walker, H. \& Small, J. Motivational Interviewing to Address Challenging Behavior: School-based Applications with teachers. Chapter in; McNamara, E., Ed. Motivational Interviewing to Address Social Issues in Schools. To be submitted June 2012. 


\section{Training \& Program Manuals}

Lee, J. (2005) Building Readers: A guide for childcare providers. Verizon Literacy Campus On-line Courses. Verizon and the National Center for Family Literacy.

Kelly, K., Fulton, J., Lee, J., McMaster, J., \& Price, G. (2003) Children's Education in Family Literacy - Preschool Impact Training for Family Literacy Professionals. The National Center for Family Literacy.

Fulton, J., Kelly, K., Lee, J., McMaster, J., \& Price, G. (2003) Adult Education in Family Literacy - ABE Reading Instruction Impact Training for Family Literacy Professionals. The National Center for Family Literacy.

\section{Funded Grants}

Frey, A., Walker, H. (2009). U.S. Department of Education, Institute of Education Sciences: R324A080137. Enhanced First Step to Success intervention for children with behavior problems.

Budget period: July 1, 2009-June 30, $2012(\$ 1,497,849)$. This is a goal 2, development project to develop, implement, and evaluate an enhanced version of the First Step intervention for children in grades K-3.

Role: Research Manager and Interventionist.

Walker, H., Feil, E., Frey, A. (2008). Eunice Kennedy Shriver National Institute of Child Health \& Human Development: 1R01HD055334-01 A2. First Step to Success intervention for young children with behavior problems

Budget Period: December 1, 2008-November 20, 2013 (The Oregon Research Institute: $\$ 3,013,692$. University of Louisville: $\$ 947,666$ ). This is an efficacy study of the First Step to Success intervention for use in early childhood settings.

Role: Consultant.

Delano, M., Lee, J. (2008). The Use of Dialogic Reading to Increase Communication and Shared Attention in Children with Autism. IRB Tracking Number 07.0261 (\$5,000). University of Louisville, KY.

Budget Period: January 1, 2008-December 31, 2008. The purpose of the study was to evaluate the effects of dialogic reading on the communication and shared attention skills of young children with ASD.

Role: Co-Investigator. 
Delano, M. (2008). The Use of Video Modeled Social Stories ${ }^{T M}$ to Improve the Social Skills of Children with Autism in Play Activities with Siblings. IRB Tracking Number $07.0260(\$ 5,000)$. University of Louisville, KY.

Budget Period: January 1, 2008-December 31, 2008. The purpose of this project was to evaluate the effects of video modeled Social Stories ${ }^{\mathrm{TM}}$ on the social engagement of children with autism spectrum disorders.

Role: Interventionist.

Lee, J. (2008). Developing Parental Support for Children's Early Language Development: An Intervention Plan. IRB Tracking Number 09.0236 $(\$ 5,000)$. Ideas to Action Grant. University of Louisville, $\mathrm{KY}$.

Budget Period: January 1, 2009-December 31,2009. The purpose of this project was to measure and monitor the language development of the youngest children served at a local shelter for the homeless, and to provide a continuing model of prevention and intervention for these children.

Lee, J., Windsor Family Literacy Program Grant. (1997-2000). Title I, Part B, Subpart 3 William F. Goodling Even Start Family Literacy Programs (Average $\$ 145,000$ per year). Windsor, CO.

Budget Period: July-June for each of four years. The purpose of this project was to develop and maintain a comprehensive family literacy program serving families with children birth to age eight in support of the adult and child's education. One main focus of the program was the provision of childcare and education for teenaged parents of the local school district.

\section{Grants Under Review}

Frey, A., Seeley, J. (Re-Submission). U.S. Department of Education, Institute of Education Sciences. Efficacy of Enhanced First Step to Success Intervention for Tertiary-Level Students with Disruptive Behavior.

Co-investigative Team: Walker, H., Feil, E., Golly, A., Small, J., Lee, J. Budget Period: July 1, 2013-June 30, 2017. The purpose of this project is to conduct a Goal 3 efficacy study to determine how potent the Enhanced First Step (EFS) intervention is for improving social/behavioral and academic outcomes to support learning, and to identify the utility of the intervention, or the degree to which it is feasible and practical for implementation in schools.

\section{Grants Under Development}

Lee, J., Frey, A. U.S. Department of Education, Institute of Education Sciences. Research Training Program in Special Education: Early Career Development and Mentoring. 
Budget Period: July 1, 2013-June 30, 2017. The purpose of this project is to develop a model for K-5 itinerant \& resource special education teachers, utilizing the First Step to Success intervention as a positive behavioral support framework and Motivational Interviewing to motivate teachers to adopt evidenced based practices. To this end, an iterative process of development, pilot testing and refinement of the measurement protocols, training materials and implementation procedures will be undertaken to (a) determine if the intervention was implemented with fidelity, (b) understand if key stakeholders perceived the intervention as socially valid, (c) better understand if the intervention functioned as intended.

\section{Unfunded Grant Submissions}

Wagner, M., Woodbridge, M. (PI, SRI International); Woodbridge, M. (Co-PI), Slaton, E., (Co-Investigator, National Federation of Families for Children's Mental Health); Seeley, J., Walker, H. (Co-Investigators, Oregon Research Institute); Frey, A. (Co-Investigators, University of Louisville). U.S. Department of Education, Institute of Education Sciences. The Research and Development Center on Interventions for Families of Students with Emotional and Behavioral Disorders - Subgrantee Application to SRI International.

Budget Period: July 1, 2012-June 30, 2017. This center (RFA 84.324C) will develop and test the efficacy of a family-centered intervention that has the potential to impact academic and behavior outcomes of students with or at risk of emotional and behavioral disorders (EBD).

Role: To be determined upon next academic appointment.

\section{Presentations}

Frey, A., Lee, J. (January, 2012) Enhanced First Step to Success. Society for Social Work and Research, Washington, DC.

Frey, A., Small, J., Lee, J., Feil, E., Seeley, J. Walker, H., Golly, A, \& Cloud, R. (2011). Enhanced homeBase. Promoting School Mental Health. Charleston, SC.

Lee, J. (2011). The First Step to Success and the Promise of Motivational Interviewing. National Conference on Family Literacy, Louisville, KY.

Frey, A., Small, J, Lee, J., Feil, E., Seeley, J. Walker, H., Golly, A, \& Cloud, R. (2010). Development of the Enhanced First Step to Success intervention. Fifth annual IES Research Conference. Washington, DC.

Lee, J. (2009). Developing Parental Support for Children's Early Language Development: Monitoring and Intervention at a Local Family Shelter. National Center for Family Literacy National Conference. Louisville, KY. 
Lee, J. (2008). Developing Parental Support for Children's Early Language Development: A Plan for Monitoring and Intervention. National Association for the Education of Homeless Children and Youth. Arlington, VA.

Rightmyer, E., Nunnelley, J., Lee, J. (2007). The Impact of Community Initiatives on School Readiness in Child Care Centers Serving Low-Income Families. National Center for Family Literacy National Conference. Orlando, FL.

\section{Courses Taught}

\section{Bellarmine University}

EDUC 582 Early Education for Exceptional Children

EDUC 315/515 Speech and Language Development

EDUC 600 Quantitative/Qualitative Research Methodology

EDUC 514 Nature and Needs of Children with LBD

EDUC 533 \& 618 Module I, MAT Program (Advanced Child Development \&

Foundations of Education)

EDUC 622 Emergent Literacy

\section{University of Louisville}

EDTP 501 General Methods

EDTP 436/536 Theories of Play

EDTP 437 Infant Toddler Development and Care

EDAP 694 Emergent Literacy

EDSP 483/683 Early Childhood Screening and Assessment 